\title{
Vector measure orthogonal sequences in spaces of square integrable functions
}

Eduardo Jiménez Fernández 


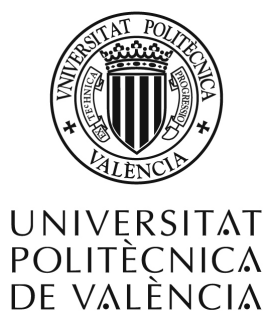

Departament de Matemàtica Aplicada Universitat Politècnica de València

\section{VECTOR MEASURE ORTHOGONAL SEQUENCES IN SPACES $\mathrm{OF}$ SQUARE INTEGRABLE FUNCTIONS}

\section{Ph.D. Dissertation}

Author

Eduardo Jiménez Fernández

Supervisors

Dr. Enrique Alfonso Sánchez Pérez

Dr. Luís Miguel García Raffi

November 2011 
(c) Eduardo J iménez Fernández, 2012

First Edition, 2012

(c) of the present edition:

Editorial Universitat Politècnica de València

www.editorial.upv.es

ISBN: 978-84-8363-806-4 (printed version)

Ref. editorial: 5518

Queda prohibida la reproducción, distribución, comercialización, transformación, y en general, cualquier otra forma de explotación, por cualquier procedimiento, de todo o parte de los contenidos de esta obra sin autorización expresa y por escrito de sus autores. 
Don Enrique Alfonso Sánchez Pérez, y Don Luís Miguel García RAFFI, profesores del Departamento de Matemática Aplicada de la Universidad Politécnica de Valencia,

CERTIFICAN que la presente memoria Vector measure orthogonal sequences of spaces of square integrable functions ha sido realizada bajo su dirección en el Departamento de Matemática Aplicada de la Universidad Politécnica de Valencia, por EduARdo Jiménez Fernández y constituye su tesis para optar al grado de Doctor en Ciencias Matemáticas.

Y para que así conste, en cumplimiento de la legislación vigente, presentamos ante el Departamento de Matemática Aplicada de la Universidad Politécnica de Valencia, la referida Tesis Doctoral, firmando el presente certificado.

En Valencia, a 27 de septiembre 2011

Fdo. Enrique Alfonso Sánchez Pérez

Fdo. Luís Miguel García Raffi 
Después de un largo periplo, presento esta memoria que es el fruto de años de trabajo y que finalmente concluyo no sin antes agradecer profundamente a mis directores Enrique A. Sánchez Pérez y Luis M. García Raffi todo el apoyo recibido en la realización de este trabajo. Quiero dar las gracias en especial al profesor José M. Calabuig Rodríguez y a la profesora María Aranzazu Juan, por sus consejos y observaciones que han sido de gran utilidad.

Finalmente quiero expresar mi gratitud, a mi esposa Rosa que ha sufrido directamente todas mis divagaciones, y a mis padres y hermana que desde el primer día me ofrecieron todo su apoyo y ánimo. 
To María 


\section{Resum}

Aquesta tesi doctoral s'emmarca dins de l'anàlisi dels subespais de successions ortogonals de funcions de quadrat integrable respecte d'una mesura vectorial que és numeràblement aditiva i pren valors en un espai de Banach. La motivació d'aquest treball és la generalització dels arguments geomètrics que proporcionen els procediments clàssics d'aproximació als espais de Hilbert. La noció d'ortogonalitat representa un punt clau que permet el desenvolupament de la teoria de la convergència de successions en aquests espais. Actualment, la convergència gairebé per a tot punt, la convergència en norma i la convergència feble són temes ben coneguts en la teoria d'espais de funcions de Hilbert.

Els espais de Banach de funcions $L^{2}(\mathbf{m})$ d'una mesura de vectorial $\mathbf{m}$ representen una àmplia classe de reticles de Banach: cada reticle de Banach 2convex ordre continu amb una unitat feble pot ser representat (a través d'un isomorfisme d'ordre) com un espai $L^{2}(\mathbf{m})$ de una mesura vectorial adequada $\mathbf{m}$. L'estructura integral que l'operador integració proporciona en aquests espais permet generalitzar arguments de ortogonalitat de la teoria d'espais de Hilbert, tot i que els espais $L^{2}(\mathbf{m})$ estan lluny de ser espais de Hilbert.

En el primer capítol d'aquesta memòria s'introdueixen alguns conceptes bàsics dels espais de Banach de funcions, integració sobre mesures vectorials i altres temes que seran necessaris al llarg de tot el treball. Es desenvolupen alguns resultats sobre la convergència de successions en espais de Banach de funcions, igual que es mostren alguns procediments que seran de gran utilitat. Alguns arguments sobre ortogonalitat també són introduits, tant en el context de succesions de $L^{2}(\mathbf{m})$ com en les integrals d'aquestes successions quan la mesura vectorial $\mathbf{m}$ pren valors en un espai de Hilbert $H$. S'analitza la convergència incondicional de successions des del punt de vista abstracte dels espais de funcions integrables, i és proporciona una versió del mètode de disjuntificació de Kadec i Pelczynsky per a mesures vectorials.

En el segon capítol, es presenten formalment tres nocions d'ortogonalitat d'una successió respecte d'una mesura vectorial. La $\mathbf{m}$-ortogonalitat feble, la (natural) $\mathbf{m}$-ortogonalitat i la $\mathbf{m}$-ortogonalitat forta, proporcionant també alguns exemples que mostren la relació amb problemes clàssics del anàlisi funcional. També s'estudia la geometria d'aquestes successions. 
En el capítol 3 analitzem la convergència gairebé per a tot punt de successions que són febles $\mathbf{m}$-ortogonals. El resultat més rellevant d'aquesta secció ens mostra una versió general del teorema de Menchoff-Rademacher. A continuació es mostra un cas particular que involucra les $c_{0}$-sumes d'un espai de Hilbert amb la finalitat de mostrar les propietats de la convergència gairebé per a tot punt.

Finalment, al capítol 4 es desenvolupa una aplicació concreta en el context de les mesures vectorials. Es facilita un mètode d'aproximació respecte d'una mesura paramètrica. Els principals elements d'aquest procediment són una successió feble $\mathbf{m}$-ortonormal i una funció integrable Bochner que definirà la nostra mesura, sobre la qual podrem calcular uns coeficients de Fourier - que en aquest cas seran funcions mesurables - per una determinada funció de $L^{2}(\mathbf{m})$. I finalment, es mostraran algunes aplicacions d'aproximació de senyals procedents de dades experimentals en el camp de l'acústica. 


\section{Resumen}

Esta tesis doctoral se enmarca dentro del análisis de los subespacios de sucesiones ortogonales de funciones de cuadrado integrable respecto de una medida vectorial que es numerablemente aditiva y toma valores en un espacio de Banach. La motivación de este trabajo es la generalización de los argumentos geométricos que proporcionan los procedimientos clásicos de aproximación en los espacios de Hilbert. La noción de ortogonalidad representa un punto clave que permite el desarrollo de la teoría de la convergencia de sucesiones en estos espacios. Hoy en día, la convergencia casi por todas partes, la convergencia en norma y la convergencia débil son temas bien conocidos en la teoría de espacios de funciones de Hilbert.

Los espacios de Banach de funciones $L^{2}(\mathbf{m})$ de una medida de vectorial $\mathbf{m}$ representan una amplia clase de retículos de Banach: cada retículo de Banach 2-convexo orden continuo con una unidad débil puede ser representado (a través de un isomorfismo de orden) como un espacio $L^{2}(\mathbf{m})$ para una medida vectorial adecuada $\mathbf{m}$. La estructura integral que el operador integración proporciona en estos espacios permite generalizar argumentos de ortogonalidad de la teoría del espacios de Hilbert, a pesar de que los espacios $L^{2}(\mathbf{m})$ están lejos de ser espacios de Hilbert.

En el primer capítulo de esta memoria se introducen algunos conceptos básicos de los espacios de Banach de funciones, integración sobre medidas vectoriales y otros temas que serán necesarios a lo largo de todo el trabajo. Se desarrollan algunos resultados sobre la convergencia de sucesiones en espacios de Banach de funciones, al igual que se muestran algunos procedimientos que serán de gran utilidad. Algunos argumentos sobre ortogonalidad también son introducidos, tanto en el contexto de sucesiones de $L^{2}(\mathbf{m})$ como en las integrales de estas sucesiones cuando la medida vectorial $\mathbf{m}$ toma valores en un espacio de Hilbert $H$. Se analiza la convergencia incondicional de sucesiones desde el punto de vista abstracto de los espacios de funciones integrables, y se proporciona una versión del método de disyuntificación de Kadec y Pelczynsky para medidas vectoriales.

En el segundo capítulo, se presentan formalmente tres nociones de ortogonalidad de una sucesión respecto de una medida vectorial. La $\mathbf{m}$-ortognalidad 
débil, la (natural) $\mathbf{m}$-ortogonalidad y la $\mathbf{m}$-ortogonalidad fuerte, proporcionando también algunos ejemplos que muestran la relación con problemas clásicos del análisis funcional. También se estudia la geometría de estas sucesiones.

En el capítulo 3 analizamos la convergencia casi por todas partes de sucesiones que son débil $\mathbf{m}$-ortogonales. El resultado más relevante de esta sección nos muestra una versión general del teorema de Menchoff-Rademacher. A continuación se muestra un caso particular que involucra las $c_{0}$-sumas de un espacio de Hilbert con el fin de mostrar las propiedades de la convergencia casi por todas partes.

Finalmente, en el capítulo 4 se desarrolla una aplicación concreta en el contexto de las medidas vectoriales. Se facilita un método de aproximación respecto de una medida paramétrica. Los principales elementos de este procedimiento son una sucesión débil $\mathbf{m}$-ortonormal y una función integrable Bochner que definirá nuestra medida, sobre la cual podremos calcular unos coeficientes de Fourier que en este caso serán funciones medibles- para una determinada función de $L^{2}(\mathbf{m})$. Y por último, se mostrarán algunas aplicaciones de aproximación de señales procedentes de datos experimentales en el campo de la acústica. 


\section{Summary}

This doctoral thesis is devoted to the analysis of orthogonal sequences in subspaces of spaces $L^{2}(\mathbf{m})$ of square integrable functions with respect to a Banach space valued countably additive measure $\mathbf{m}$. The motivation of our work is to generalize the geometric arguments that provide the classical approximation procedures in Hilbert spaces. The notion of orthogonality lies in the center of the Hilbert space theory, and it allows to develop the theory of convergence of sequences in these spaces. Almost everywhere convergence, norm convergence and weak convergence are nowadays well known topics in the Hilbert space function theory.

The Banach function spaces $L^{2}(\mathbf{m})$ of a vector measure $\mathbf{m}$ represent a broad class of Banach lattices: each 2-convex order continuous Banach lattice with a weak unit can be represented (by means of an order isomorphism) as a space $L^{2}(\mathbf{m})$ for an adequate vector measure $\mathbf{m}$. The integral structure that the vector measure integration provides in these spaces allows to generalize the orthogonality arguments of the Hilbert space theory, although the spaces $L^{2}(\mathbf{m})$ are far from being Hilbert spaces.

The first chapter of this memoir is devoted to introduce some fundamental concepts on Banach function spaces, vector measure integration and other topics that will be necessary in the rest of the work. Some results on convergence of sequences in Banach function spaces and Banach spaces are explained, and the general framework is established. Some orthogonality arguments are already introduced, both for sequences in $L^{2}(\mathbf{m})$ and for the integrals of these sequences when the vector measure $\mathbf{m}$ is Hilbert space valued. Unconditional convergence for sequences from the abstract point of view of the function spaces of integrable functions is analyzed, and a version of the Kadec and Pelczynsky method for finding disjoint sequences for the vector measure setting is given.

In the second chapter three notions of orthogonality of a sequence with respect to a vector measure are formally introduced, and the main characterizations of these sequences are given. Weak $\mathbf{m}$-orthogonal sequences, (natural) $\mathbf{m}$-orthogonal sequences and strongly $\mathbf{m}$-orthogonal sequences are defined and studied, providing also examples that show the relation with some classical prob- 
lems in analysis. The geometry of these sets of sequences are also studied.

In Chapter 3 we analyze almost everywhere convergence of weak $\mathbf{m}$-orthogonal sequences. Our main result is a general vector measure version of the MenchoffRademacher Theorem. A particular case involving $c_{0}$-sums of Hilbert spaces is also intensively studied in order to show the properties of the convergence.

Finally, Chapter 4 is devoted to show a concrete application. We develop an approximation method with respect to a parametric measure based on our ideas. A Bochner integrable function and an weak $\mathbf{m}$-orthonormal sequence are the main elements of our procedure, that allows to find the Fourier coefficients -that are in this case measurable functions- for a given function in the space $L^{2}(\mathbf{m})$. Some applications for signal approximation for data coming from experimental acoustics are also shown. 


\section{Contents}

0.1 Motivation ..................... . . i

0.2 Notes and remarks ..................... .... ii

0.2.1 Moment's problem ............... in

0.2.2 $L^{2}(\mathbf{m})$ spaces from the point of view of the vector measure theory .................. iv

0.3 Applications ....................... . . . v

0.4 The structure of the thesis . . . . . . . . . . . . . . . . . vi vi

1 Notation and Preliminaries 1

1.1 Basic notions . . . . . . . . . . . . . . . . . . . 1

1.2 Unconditional basis in $L^{2}(\mathbf{m})$. . . . . . . . . . . . . . . . . . . 7

1.3 Kadec-Pelczyński decomposition . . . . . . . . . . . . 13

2 m-Orthogonal sequences with respect to a vector measure 19

$2.1 \mathbf{m}$-Orthogonal sequences of functions with respect to a vector measure ..................... 19

2.2 Weak m-orthogonal sequences . . . . . . . . . . . . . . . . . . 20

$2.3 \mathbf{m}$-Orthogonal sequences .................. 25

2.4 Strongly $\mathbf{m}$-orthogonality . . . . . . . . . . . . . . . . . . 31

3 The Menchoff-Rademacher Theorem for $L^{2}(\mathbf{m}) \quad 39$

3.1 About almost everywhere convergence of sequences in $L^{2}(\mathbf{m})$. . 41

3.2 The Menchoff-Rademacher Theorem for weak m-orthogonal sequences . . . . . . . . . . . . . . . . . . . . 44

3.3 Almost everywhere convergence in $c_{0}$-sums of $L^{2}(\mu)$ spaces $\ldots . \quad 50$

4 Pointwise dependent Fourier coefficients $\quad \mathbf{5 3}$

4.1 Pointwise dependent Fourier coefficients . . . . . . . . . . . . . . 54

4.2 Continuity of the pointwise dependent Fourier coefficients . . . . . . . . . . . . . . . 62

4.3 An example of approximation with finite $\mathbf{m}$-orthogonal sequences 65

4.4 Application to acoustic data . . . . . . . . . . . . . 72

$\begin{array}{ll}\text { References } & 77\end{array}$ 


\section{Introduction}

\subsection{Motivation}

The notion of orthogonality lies in the center of the Hilbert and Banach spaces theory and it has a clear geometrical meaning. Even for the case of finite dimensional spaces with the Euclidean norm the notion of orthogonality is deeply connected with the topological properties of the space and mainly with the notion of best approximation. The same relation can be extended to the setting of the Hilbert spaces, which has as a canonical example the space $L^{2}[0,1]$. In the year 2000, a new class of Banach function spaces was introduced: the spaces $L^{p}(\mathbf{m})$ of $p$-integrable functions with respect to a vector measure $\mathbf{m}$. Those spaces are rather general, since they represent the class of all order continuous $p$-convex Banach function spaces with a weak unit. So, the space $L^{2}(\mathbf{m})$ is 2 convex, but it is far of having a Hilbert space structure. However, and due to its integration structure, several notions of orthogonality still make sense in it. The geometric consequences of these notions and some applications in the context of the best approximation and Fourier analysis has been studied in recent years, as the reader can notice by checking the references in this memoir.

The aim of this thesis is to analyze and to show some applications of the three notions of vector measure orthogonality that has been introduced in the literature up to this moment, and to develop a systematic theory of vector measure orthogonality including all these cases. This of course imply to study the structure of the spaces $L^{2}(\mathbf{m})$ of a vector measure and the main properties 
of the integration map restricted to these spaces.

One of the basic concepts that leads to the one of vector measure orthogonality is the vector measure duality. It consists on considering the duality induced by the bilinear form defined by the integration map, i.e. if $\mathbf{m}: \Sigma \rightarrow X$ is a countably additive vector measure with values in the Banach space $X$ and $1=1 / p+1 / q$, the integral defines a bilinear form $B_{m}: L^{p}(\mathbf{m}) \times L^{q}(\mathbf{m}) \rightarrow X$ by $B_{\mathbf{m}}(f, g):=\int f g d \mathbf{m}$. Duality results can be found in [30,31, 59,60,61]. These duality results, that provide for instance a representation of the dual space, are applied in this thesis in the symmetric case given by $p=2$.

\subsection{Notes and remarks}

In this section we are going to show the concept of orthogonality with respect to a vector measure has its roots in the $X I X$ century.

\subsubsection{Moment's problem}

The orthogonality with respect to a vector measure can be easily related to some classical mathematical problems, as the so called Moment's problem. Actually, as will be shown in Chapter 2, orthogonality with respect to a vector measure is a general setting that includes for instance orthogonality of a sequence of functions with respect to a family of measures, or what is called in statistics, a parametric measure. The analysis of the properties of functions that are orthogonal with respect to a family of measures has a long mathematical history, for instance regarding orthogonal polynomials. In 1885 Tchebyshev established the following question. If the relations

$$
\int_{-\infty}^{\infty} x^{n} \omega(x) d x=\int_{-\infty}^{\infty} x^{n} e^{-x^{2}} d x
$$

holds for all $n \in \mathbb{N}$ this implies that $\omega(x)=e^{-x^{2}}$ ? The answer is yes and today we say that the problem is determinate. 
There are different points of view to try to solve this problem. The first procedure was developed by Stieljes in 1894 (see [62]). According Dieudonné (see [26]), the moment's problem is no stranger to the birth of Banach theory. M. Riesz in [52] provided a solution using the Helly's Representation Theorem (see [13]), and Nevalinna introduced a new tool into the analytic function theory in [4] for trying to solve this problem.

When the problem is not determined we obtain a family of scalars measures such that $\int_{-\infty}^{\infty} x^{n} \omega(x) d x=S_{n}, \quad n=0,1,2 \ldots$ have the same moments. At the end of the 19th century some relevant cases of families of polynomials that are orthogonal with respect to a large set of scalar measures -indeterminate measures - were known. The first example of such an indeterminate measure was presented by Stieltjes in 1894 (see [62]). If we start with the two following integrals

$$
\int_{0}^{\infty} e^{-t^{2}} d t=\sqrt{\pi} \quad \text { and } \quad \int_{0}^{\infty} e^{-t^{2}} \sin (2 \pi t) d t=0
$$

and now we do the next transformation $t=\ln (x)-(n+1) / 2$, then

$$
e^{-t^{2}}=e^{-\ln ^{2}(x)} x^{n+1} e^{-(n+1)^{2} / 4} \quad \text { and } \quad d t=\frac{d x}{x} .
$$

So we obtain

$$
\int_{0}^{\infty} x^{n} e^{-\ln ^{2}(x)} d x=\sqrt{\pi} e^{(n+1)^{2} / 4}, \quad \int_{0}^{\infty} x^{n} e^{-\ln ^{2}(x)} \sin (2 \pi \ln (x)) d x=0 .
$$

Then if we multiply the second integral by a constant $K \in \mathbb{R}$ and we add the first integral, we obtain

$$
\int_{0}^{\infty} e^{-\ln ^{2}(x)} x^{n}[1+K \sin (2 \pi \ln (x))] d x=\sqrt{\pi} e^{(n+1)^{2} / 4} .
$$

If we take $|K|<1$ then $e^{-\ln ^{2}(x)}[1+K \sin (2 \pi \ln (x))]>0$ is a positive function for all $x \in\left[0, \infty\left[\right.\right.$, thus $F_{K}(y)=\int_{0}^{y} e^{-\ln ^{2}(x)} x^{n}[1+K \sin (2 \pi \ln (x))] d x$ is a family of non decreasing distributions with support into $[0, \infty[$ which have the same moments $\sqrt{\pi} e^{(n+1)^{2} / 4}$. The polynomials that are orthogonal with respect to this class of measures are a special case of the Stieltjes-Wigert polynomials. Using the family $\left(F_{K}\right)_{K \in[-1,1]}$ of distributions, a vector measure can be defined in an easy way (see Example 2.3.2). In general this construction can be done for abstract sets of measures - for instance, parametric models in statistics-, and then to find sequences of functions that are orthogonal for all the elements 
of a family of measures is equivalent to the problem of finding sequences that are orthogonal with respect to a vector measure.

Our main references on measure spaces, scalar measures, integration with respect to scalar measures, vector measures and function spaces are $[25,33$, $42,49]$. The reader can find general definitions and results on integration with respect to vector measures and the related spaces of $\mathbf{m}$-integrable functions $L^{1}(\mathbf{m})$ in $[15,40,41]$.

\subsection{2 $L^{2}(\mathrm{~m})$ spaces from the point of view of the vector measure theory}

From the point of view of the integration with respect to a vector measure, the properties of the integration map that are nowadays well known provide relevant information with important consequences on the properties of the orthogonal sequences in the spaces $L^{2}(\mathbf{m})$. Compactness properties of the integration map - weak compactness, compactness, complete continuity - have direct consequences on the structure of the corresponding spaces of integrable functions. For instance, if the integration operator is compact, the corresponding space is order isomorphic to the $L^{1}$-space of the variation $|\mathbf{m}|$, i.e. an $L^{1}$-space. Consequently, in this case $L^{2}(\mathbf{m})$ is order isomorphic to the Hilbert space $L^{2}(|\mathbf{m}|)$ (see Proposition 3.48 in [49] and the references therein). Regarding weak compactness, it is also known that the integration map restricted to $L^{2}(\mathbf{m})$ is always weakly compact (see $[28,29,60])$. Also, the geometric properties of the integration map will be used in this thesis. For instance, $p$-concavity of the integration map restricted to $L^{p}(m)$ implies that this space is order isomorphic to an $L^{p}$ space (see $[9,16,17])$.

In recent years, some applications of the spaces $L^{2}(\mathbf{m})$ have been developed in the setting of the function approximation. In particular, the geometry of the strongly $\mathbf{m}$-orthogonal sequences is well known and can be found in the papers $[34,48,59]$. Some applications on function approximation of $\mathbf{m}$-orthogonal sequences were developed also in the papers [34, 35, 36]. 
Some results that follows the general research program of analyzing convergence of sequences in the spaces $L^{p}$ of a vector measure has been published yet, and do not constitute a part of this memoir, although they are closely related. In this direction, we must mention two papers. The first one, [10], analyzes and improves some known results on decompositions of unconditional convergence of sequences in Banach function spaces using the structure of the spaces $L^{p}(\mathbf{m})$. The second one, [37], provides a general analysis of the Komlós property regarding a.e. convergence of the Cesàro sums in spaces of measurable functions. In this case, a most general theory of vector measure integration involving $\delta$ rings is used in order to establish how far, in the scale of ideals of measurable functions, the Fatou property and the Komlós property are equivalent.

Those results have not being included in this memoir because they do not use any orthogonality argument, although the subject that they deal with is very much concerned with the one of the work presented in this memoir: different aspects of the convergence of sequences in the spaces $L^{2}$ of a vector measure.

\subsection{Applications}

The last part of the thesis (Chapter 4) is devoted to show some new applications of the spaces $L^{2}(\mathbf{m})$ in the setting of the function approximation. In particular, we develop an approximation structure that consists of providing a parametric set of measures by means of the action of a Bochner integrable function with the vector measure. A suitable error criterion is defined and the corresponding approximation formulae are given. This leads to a non linear approximation for a given function of $L^{2}(\mu)$, that has the advantage that a small orthogonal set of functions is enough for obtaining a good approximation, improving the one that is obtained when the Hilbert space structure is used. Some examples are given, and a concrete application to representation of acoustic signals is developed. 


\subsection{The structure of the thesis}

Chapter 1 is devoted to recall and adapt some well known results on the behavior of sequences in Banach function spaces. An adapted version of the BessagaPelczynski for weakly null sequences (see [7]) and the Kadec-Pelczynski method for obtaining disjoint sequences that approximate special sequences is given (see $[32,38])$.

In Chapter 2, the three notions of $\mathbf{m}$-orthogonality are given and analyzed, showing some examples and existence results for such sequences in the $L^{2}(\mathbf{m})$ spaces.

Chapter 3 shows how the Menchoff-Rademacher results on almost everywhere convergence of sequences can be adapted and improved for the case of $\mathbf{m}$-orthogonal sequences, that comes from the fact that the vector measure orthogonality is stronger than the scalar notion. The last chapter provides the applications of the theory that has been explained above. 


\section{Chapter 1}

\section{Notation and Preliminaries}

\subsection{Basic notions}

In this chapter, we introduce the concepts and results used throughout the memory about Banach function spaces and integration of real functions with respect to a vector measure. We will use standard Banach and function space notation; our main references are $[25,42,64]$. Let $X$ be a Banach space. We will denote by $B_{X}$ the unit ball of $X$, that is $B_{X}:=\{x \in X:\|x\|=1\} . X^{\prime}$ is the topological dual of $X$ and $B_{X^{\prime}}$ its unit ball. If $1 \leq p \leq \infty$, we write $q$ for the (extended) real number satisfying $1 / p+1 / q=1$. A Banach space $X$ is said of type $p$ for some $1<p \leq 2$ respectively, of cotype $q$ for some $q \geq 2$, if there exists a constant $M<\infty$ so that, for every finite set of vectors $\left\{x_{j}\right\}_{j=1}^{n}$ in $X$, we have

$$
\int_{0}^{1}\left\|\sum_{i=1}^{n} r_{j}(t) x_{j}\right\| d t \leq M\left(\sum_{j=1}^{n}\left\|x_{j}\right\|^{p}\right)^{1 / p},
$$

respectively

$$
\int_{0}^{1}\left\|\sum_{i=1}^{n} r_{j}(t) x_{j}\right\| d t \geq \frac{1}{M}\left(\sum_{j=1}^{n}\left\|x_{j}\right\|^{q}\right)^{1 / q},
$$

where $\left\{r_{j}\right\}_{j}$ denotes the sequence of the Rademacher functions. The Hilbert spaces have the best possible type and cotype, i.e. are simultaneously of type 2 
and cotype 2 and the converse of this assertion is also true (see $[42,1 . e .12]$ ). Let $X$ and $Y$ be Banach spaces. An operator $T: X \rightarrow Y$ is 2-absolutely summing if there exists a constant $C>0$ such that for every finite sequence $x_{1}, \ldots, x_{n} \in X$,

$$
\left(\sum_{i=1}^{n}\left\|T\left(x_{i}\right)\right\|^{2}\right)^{\frac{1}{2}} \leq C \sup \left\{\left(\sum_{i=1}^{n}\left|\left\langle x_{i}, x^{\prime}\right\rangle\right|^{2}\right)^{\frac{1}{2}}: x^{\prime} \in X^{\prime},\left\|x^{\prime}\right\| \leq 1\right\} .
$$

We define the 2-summing norm of $T$ as

$$
\pi_{2}(T)=\inf \left\{C:(1.1) \text { holds for all }\left\{x_{i}\right\}_{i=1}^{n} \subset X, \quad n \in \mathbb{N}\right\} .
$$

Let $X$ be a Banach lattice, that is a real Banach space endowed with a norm $\|\cdot\|$ and a partial order $\leq$ such that

(1) if $x, y, z \in X$ with $x \leq y$, then $x+z \leq y+z$,

(2) if $x, y \in X$ with $x \leq y$, then $\alpha x \leq \alpha y$ for all $\alpha>0$,

(3) for $x, y \in X$, there exists the supremum of $x$ and $y$ with respect to the order,

(4) if $x, y \in X$ with $|x| \leq|y|$, then $\|x\| \leq\|y\|$, where $|x|=\sup \{x,-x\}$ is the modulus of $\mathrm{x}$.

Note that (3) implies that also there exists the infimum of every $x, y \in X$. The supremum and the infimum of two elements $x$ and $y$ of $X$ are usually denoted by $x \vee y$ and $x \wedge y$ respectively. A weak unit of $X$ is an element $0 \leq e \in X$ such that $x \wedge e=0$ implies that $x=0$.

We say that a Banach lattice $X$ is order continuous if for every sequence $\left\{x_{n}\right\}_{n} \subset$ $X$ with $x_{n} \downarrow 0$ it follows that $\left\|x_{n}\right\| \downarrow 0$. We say that $X$ has the Fatou property if for every net $\left(x_{\tau}\right) \subset X$ with $0 \leq x_{\tau} \uparrow$ such that $\sup \left\|x_{\tau}\right\|<\infty$ if follows that there exists $x=\sup x_{\tau}$ in $X$ and $\|x\|=\sup \left\|x_{\tau}\right\|$. Let $T: X \rightarrow Y$ be a linear operator between two Banach lattices. The operator $T$ is said to be positive if $T(x) \geq 0$ whenever $0 \leq x \in X$. Every positive linear operator between two Banach lattices is continuous. We will say that $T$ is an order isomorphism if it is one to one, onto and satisfies that $T(x \wedge y)=T(x) \wedge T(y)$ for all $x, y \in X$. In this case, $T$ is continuous as it is positive and also satisfies $T(x \vee y)=T(x) \vee T(y)$ for all $x, y \in X$. If moreover, $\|T(x)\|_{Y}=\|x\|_{X}$ for all $x \in X$, we will say that $T$ is an order isometry. Let $E$ and $F$ be Banach lattices and $1 \leq p<\infty$. An operator $T: E \rightarrow F$ is $p$-concave if there is a constant $K>0$ such that for 
every finite set $x_{1}, x_{2}, \ldots, x_{n} \in E$, it follows

$$
\left(\sum_{i=1}^{n}\left\|T\left(x_{i}\right)\right\|^{p}\right)^{\frac{1}{p}} \leq K\left\|\left(\sum_{i=1}^{n}\left|x_{i}\right|^{p}\right)^{\frac{1}{p}}\right\| .
$$

The infimum of such constants $K$ is the $p$-concavity constant of the operator. An operator $T: E \rightarrow F$ is $p$-convex if there is a constant $K>0$ such that for each finite set $x_{1}, x_{2}, \ldots, x_{n} \in E$, it follows

$$
\left\|\left(\sum_{i=1}^{n}\left|T\left(x_{i}\right)\right|^{p}\right)^{\frac{1}{p}}\right\| \leq K\left(\sum_{i=1}^{n}\left\|x_{i}\right\|^{p}\right)^{\frac{1}{p}} .
$$

As in the case of $p$-concavity, the infimum of such constants $K$ is the $p$-convexity constant of $T$. A Banach lattice $E$ is $p$-concave ( $p$-convex) if the identity map $I d: E \rightarrow E$ is $p$-concave ( $p$-convex). Throughout the memoir we will consider Banach function spaces as Banach lattices with the usual $\mu$-a.e. pointwise order. For the aim of simplicity, we will assume that the corresponding $p$-concavity $/ p$ convexity constants of the spaces are 1 ; it is known that each $r$-convex and $s$-concave Banach lattice, $1 \leq r \leq s \leq \infty$, can be renormed equivalently so that with the new norm, the $r$-convexity and $s$-concavity constants are both equal to 1 (see $[42,1$. d. 8$]$ ).

Let $(\Omega, \Sigma, \mu)$ be a $\sigma$-finite measure space. Following the definition in [42, p.28], a Banach space $X(\mu)$ of (classes of) locally $\mu$-integrable real functions is said to be a Banach function space over $\mu$ (Köthe function space) if it satisfies the next two properties.

- If $f$ is measurable and $g \in X(\mu)$ such that $|f(w)| \leq|g(w)| \mu$-a.e. on $\Omega$, then $f \in X(\mu)$ and $\|f\| \leq\|g\|$.

- If $A \in \Sigma$, and $\mu(A)<\infty$, then the characteristic function $\chi_{A}$ belongs to $X(\mu)$.

We write as usual $\ell^{p}, 1 \leq p<\infty$, and $c_{0}$ for the classical sequence spaces, and $\|\cdot\|_{p},\|\cdot\|_{0}$ for the corresponding norms. The sequence spaces that we deal with $(L, \ell \ldots)$ are assumed to be such kind of spaces. Thus, we will consider spaces of real functions on the standard measure space on the set of natural numbers $\mathbb{N}$ with an unconditional normalized basis with unconditional constant 1. We will write $e_{i}, i \in \mathbb{N}$, for the elements of the canonical basis of the space. Moreover, we also assume that its dual space can be represented as a sequence space, i.e. its elements can be written as sequences $\left\{\tau_{i}\right\}_{i}$, and the duality is given by $\left\langle\left\{\tau_{i}\right\},\left\{\lambda_{i}\right\}\right\rangle=\sum_{i=1}^{\infty} \tau_{i} \lambda_{i},\left\{\lambda_{i}\right\}_{i} \in L$. For instance, this always happens 
when the space is order continuous (see the comments that follow Definition 1.b.17 in [42]). We will use the following construction for the particular case of sequence spaces (i.e. the measure is the discrete one on the set of the natural numbers). Let $(\Omega, \Sigma)$ be a measurable space and $X$ a Banach space. Throughout the memory $\mathbf{m}: \Sigma \rightarrow X$ will be a countably additive vector measure, i.e. $\mathbf{m}\left(\cup_{n=1}^{\infty} A_{n}\right)=\sum_{n=1}^{\infty} \mathbf{m}\left(A_{n}\right)$ in the norm topology of $X$ for all sequences $\left\{A_{n}\right\}_{n}$ of pairwise disjoint sets of $\Sigma$. It is well known that every Banach space valued countably additive vector measure on a $\sigma$-algebra is bounded. We say that a countably vector measure $\mathbf{m}: \Sigma \rightarrow X$, where $X$ is a Banach lattice, is positive if $\mathbf{m}(A) \geq 0$ for all $A \in \Sigma$. For each element $x^{\prime} \in X^{\prime}$ the formula $\left\langle\mathbf{m}, x^{\prime}\right\rangle(A):=\left\langle\mathbf{m}(A), x^{\prime}\right\rangle, A \in \Sigma$, defines a (countably additive) scalar measure. We write $\left|\left\langle\mathbf{m}, x^{\prime}\right\rangle\right|$ for its variation, i.e. $\left|\left\langle\mathbf{m}, x^{\prime}\right\rangle\right|(A):=\sup \sum_{B \in \Pi}\left|\left\langle\mathbf{m}(B), x^{\prime}\right\rangle\right|$ for $A \in \Sigma$, where the supremum is computed over all finite measurable partitions $\Pi$ of $A$. Sometimes we write $\mathbf{m}_{x^{\prime}}$ for $\left\langle\mathbf{m}, x^{\prime}\right\rangle$. We say that an element $x^{\prime} \in X^{\prime}$ is $\mathbf{m}$-positive if the scalar measure $\mathbf{m}_{x^{\prime}}$ is positive, i.e. $\left|\mathbf{m}_{x^{\prime}}\right|=\mathbf{m}_{x^{\prime}}$. We write $\left(X^{\prime}\right)_{\mathbf{m}}^{+}$for the set of these elements. The semivariation of $\mathbf{m}$ is the extended nonnegative function $\|\mathbf{m}\|$ whose value on a set $A \in \Sigma$ is given by:

$$
\|\mathbf{m}\|(A)=\sup \left\{\left|\left\langle\mathbf{m}, x^{\prime}\right\rangle\right|(A): x^{\prime} \in X^{\prime},\left\|x^{\prime}\right\| \leq 1\right\} .
$$

Direct computations show that the variation $|\mathbf{m}|$ is a monotone countably additive function on $\Sigma$, while the semivariation $\|\mathbf{m}\|$ is a monotone subadditive function on $\Sigma$, and for each $A \in \Sigma$ we have that $\|\mathbf{m}\|(A) \leq|\mathbf{m}|(A)$. A vector measure $\mathbf{m}$ defined on a $\sigma$-algebra is always bounded, i.e. $\mathbf{m}(A)<\infty$ for all $A \in \Sigma$. In general, a vector measure $\mathbf{m}$ is of bounded semivariation on $\Omega$ if and only if its range is bounded in $X$, as for $A \in \Sigma, \sup \{\|\mathbf{m}(B)\|: A \supseteq B \in \Sigma\} \leq$ $\|\mathbf{m}\|(A) \leq 4 \sup \{\|\mathbf{m}(B)\|: A \supseteq B \in \Sigma\}$. As usual, we say that a sequence of functions converges $\left|\left\langle\mathbf{m}, x^{\prime}\right\rangle\right|$-almost everywhere if it converges pointwise in a set $A \in \Sigma$ such that $\left|\left\langle\mathbf{m}, x^{\prime}\right\rangle\right|(\Omega \backslash A)=0$. A sequence converges $\mathbf{m}$-almost everywhere if it converges in a set $A$ that satisfies that the semivariation of $\mathbf{m}$ in $\Omega \backslash A$ is 0 .

We will say that a scalar positive measure $\mu$ is equivalent to $\mathbf{m}$ if

$$
\lim _{\mu(A) \rightarrow 0}\|\mathbf{m}\|(A)=0 \quad \text { and } \quad \lim _{\|\mathbf{m}\|(A) \rightarrow 0} \mu(A)=0 .
$$

The measure $\mathbf{m}$ is absolutely continuous with respect to $\mu$ if $\lim _{\mu(A) \rightarrow 0} \mathbf{m}(A)=$ 0 ; in this case we write $\mathbf{m} \ll \mu$ and we say that $\mu$ is a control measure for $\mathbf{m}$. Countably additive vector measures always have a control measure. The Bartle, 
Dunford and Schwartz theorem (see [25, Ch.I,2, Corollary 6]) produces a finite nonnegative real-valued measure $\mu$ on $\Sigma$ such that $\mathbf{m} \ll \mu$. Furthermore, it is known that there exists always an element $x^{\prime} \in X^{\prime}$ such that $\mathbf{m} \ll\left|\left\langle\mathbf{m}, x^{\prime}\right\rangle\right|$ and so $\mathbf{m} \ll\left|\left\langle\mathbf{m}, x^{\prime}\right\rangle\right|$ is a control measure for $\mathbf{m}$. We call such a scalar measure a Rybakov measure for $\mathbf{m}$ (see [25, Ch.IX,2]). If $\left|\left\langle\mathbf{m}, x^{\prime}\right\rangle\right|$ is a Rybakov measure for $\mathbf{m}$, then a sequence of functions converges $\mathbf{m}$-almost everywhere if and only if it converges $\left|\left\langle\mathbf{m}, x^{\prime}\right\rangle\right|$-almost everywhere. Notice that if $\mathbf{m}$ is positive and $x^{\prime}$ is a positive element of the Banach lattice $X^{\prime}$, then $\left|\left\langle\mathbf{m}, x^{\prime}\right\rangle\right|=\left\langle\mathbf{m}, x^{\prime}\right\rangle$.

The space $L^{1}(\mathbf{m})$ of integrable functions with respect to $\mathbf{m}$ is a Banach function space over any Rybakov measure $\mu$ for $\mathbf{m}$ (see $[15,42]$ ). The elements of this space are (classes of $\mu$-a.e. measurable) functions $f$ that are integrable with respect to each scalar measure $\left\langle\mathbf{m}, x^{\prime}\right\rangle$, and for every $A \in \Sigma$ there is an element $\int_{A} f d \mathbf{m} \in X$ such that $\left\langle\int_{A} f d \mathbf{m}, x^{\prime}\right\rangle=\int_{A} f d\left\langle\mathbf{m}, x^{\prime}\right\rangle$ for every $x^{\prime} \in X^{\prime}$. When no explicit reference is needed, we write $\int f d \mathbf{m}$ instead of $\int_{\Omega} f d \mathbf{m}$. The reader can find the definitions and fundamental results concerning the space $L^{1}(\mathbf{m})$ in $[15,40]$.

The space of (classes of $\mu$-a.e. equal) real measurable functions on $(\Omega, \Sigma)$ is denoted by $L^{0}(\mu)$. The formula

$$
|\|f\||_{L^{1}(\mathbf{m})}:=\sup _{A \in \Sigma}\left\|\int_{A} f d \mathbf{m}\right\|_{X}, \quad f \in L^{1}(\mathbf{m}) .
$$

where $\|\cdot\|_{X}$ denotes the norm of $X$, gives a norm on $L^{1}(\mathbf{m})$, since functions that are equal $\mathbf{m}$-a.e. are identified. Moreover,

$$
|\|f\||_{L^{1}(\mathbf{m})} \leq\|f\|_{L^{1}(\mathbf{m})} \leq 2|\|f\||_{L^{1}(\mathbf{m})} \quad \text { for every } \quad f \in L^{1}(\mathbf{m}) .
$$

The space $L^{1}(\mathbf{m})$ of $\mathbf{m}$-a.e. equal $\mathbf{m}$-integrable functions is a Banach lattice endowed with the norm $\|\cdot\|_{L^{1}(\mathbf{m})}$ and the $\mathbf{m}$-a.e. order. It is an ideal of measurable functions, that is $|f| \leq|g| \mathbf{m}$-a.e. with $f \in L^{0}(\mu)$ and $g \in L^{1}(\mathbf{m})$, then $f \in L^{1}(\mathbf{m})$ and an order continuous Banach lattice.

We build the spaces $L^{p}(\mathbf{m})$, that are also order continuous Banach function spaces over the space $\left(\Omega, \Sigma,\left|\mathbf{m}_{x_{0}^{\prime}}\right|\right)$ with weak unit where $\left|\mathbf{m}_{x_{0}^{\prime}}\right|$ is a Rybakov measure. We say that a measurable function $f: \Omega \rightarrow \mathbf{R}$ is $p$-integrable with respect to $\mathbf{m}$ if $|f|^{p} \in L^{1}(\mathbf{m})$ with the norm

$$
\|f\|_{L^{p}(\mathbf{m})}:=\left\||f|^{p}\right\|_{L^{1}(\mathbf{m})}^{\frac{1}{p}}, \quad f \in L^{p}(\mathbf{m}) .
$$


We denote the integration operator associated to the vector measure $\mathbf{m}$ by

$$
\begin{aligned}
I_{\mathbf{m}}: L^{p}(\mathbf{m}) & \longrightarrow X \\
f & \longmapsto I_{\mathbf{m}}(f)=\int_{A} f d \mathbf{m} .
\end{aligned}
$$

The properties of the integration map associated to a vector measure has been largely studied in several recent papers (see [22, 49]).

Before introducing several results concerning basic sequences, we provide a representation of the dual space of $L^{p}(\mathbf{m})$ in terms of the space $L^{q}(\mathbf{m})(\mathbf{m}$ is a countably additive vector measure), as in the case of classical $L^{p}$-spaces. It is well know that the dual of $L^{p}$ coincides with $L^{q}$ only in the case that $\mathbf{m}$ is a scalar measure. Let $\mathcal{L}\left(L^{p}(\mathbf{m}), X\right)$ be the subspace of all the operators that satisfy Theorem 2.2 [30], i.e. that can be identified (isometrically and isomorphically) with functions of $L^{q}(\mathbf{m})$. Indeed $L^{q}(\mathbf{m})$ is always the dual of a certain topological tensor product. In the same paper, the autors prove the following result: the spaces $\left(\left(\left(L^{p}(\mathbf{m}) \otimes X^{\prime}\right) / \operatorname{ker} u, \tau_{\widetilde{u}}\right)^{\prime},\|\cdot\|_{\widetilde{u}}\right)$ and $\left(L^{q}(\mathbf{m}), \| \cdot\right.$ $\left.\|_{L^{q}(\mathbf{m})}\right)$ are isometrically isomorphic if and only if the unit ball of $L^{q}(\mathbf{m})$ is $\mathbf{m}$-weakly compact, where $u(z)=\sup _{\|g\|_{L^{q}(\mathbf{m})} \leq 1}\left|\sum_{i=1}^{n}\left\langle\int f_{i} g d \mathbf{m}, x_{i}^{\prime}\right\rangle\right|$ if $z=$ $\sum_{i=1}^{n} f_{i} \otimes x_{i}^{\prime} \in L^{p}(\mathbf{m}) \otimes X^{\prime}$ and the quotient space $\left(L^{p}(\mathbf{m}) \otimes X^{\prime}\right) /$ ker $u$ define the quotient topology $\tau_{\tilde{u}}$ generated by the seminorm $\widetilde{u}([z]):=u(z)$. Since the $\mathbf{m}$-weak topology is weaker than the weak topology of the space $L^{p}(\mathbf{m})$, the compactness property required in the above result is satisfied if the space $L^{q}(\mathbf{m})$ is reflexive. Another prove of this result can be found in [31]. Some results regarding reflexivity of this space may be found in [28]. We recall that a Banach lattice $X$ is a KB-space whenever every norm bounded, positive, increasing sequence is norm convergent, then it is known that for $q>1 L^{q}(\mathbf{m})$ is reflexive if and only if $L^{q}(\mathbf{m})$ is a KB-space, and a Banach lattice is a KB space if and only if $X$ has the Fatou property and is an order continuous Banach lattice, so $L^{q}(\mathbf{m})$ is reflexive if and only if $L^{q}(\mathbf{m})$ has the Fatou property.

In this memoir we deal with sequences of functions in $L^{2}(\mathbf{m})$. If $\left\langle\mathbf{m}, x^{\prime}\right\rangle$ is a Rybakov measure for $\mathbf{m}$, then the inclusion map $i_{x^{\prime}}: L^{2}(\mathbf{m}) \rightarrow L^{2}\left(\left|\left\langle\mathbf{m}, x^{\prime}\right\rangle\right|\right)$ is well-defined and continuous; in fact, even if $x^{\prime}$ do not define a Rybakov measure this identification map is well-defined and continuous, although it is not injective. In this work we only need some particular properties of the functions in $L^{2}(\mathbf{m})$. For instance, if $f, g$ are functions in $L^{2}(\mathbf{m})$, we use the fact that the product $f g$ is $\mathbf{m}$-integrable (see $[28,49]$ or [60]). 
If $L^{0}(\mu)$ is the space of (classes of $\mu$-a.e. equal) real measurable functions, $0<r<\infty$ and $E(\mu)$ is a Banach function space, we define the $r$-power of $E(\mu)$ as the space

$$
E(\mu)_{[r]}:=\left\{x \in L^{0}(\mu):|x|^{1 / r} \in E(\mu)\right\}
$$

endowed with the (quasi-)norm $\|x\|_{E_{[r]}}:=\left\||x|^{1 / r}\right\|_{E}^{r}$. The space $E(\mu)_{[r]}$ is always a Köthe function space when $0<r \leq 1$ and for $r>1$ it is so whenever $E(\mu)$ is $r$ convex; in this case the expression above gives a norm if the $r$-convexity constant is 1 (see $[19,49]$ for the basic properties of $r$-powers of Köthe function Banach spaces). For instance, if $\mathbf{m}$ is a countably vector measure, the space $L^{2}(\mathbf{m})$ above can be written as the $1 / 2$-power of $L^{1}(\mathbf{m})$, i.e. $L^{2}(\mathbf{m})=\left(L^{1}(\mathbf{m})\right)_{[1 / 2]}$.

\subsection{Unconditional basis in $L^{2}(\mathbf{m})$}

A sequence $\left\{x_{n}\right\}_{n=1}^{\infty}$ in a Banach space $X$ is called a Schauder basis of $X$ if there exists a unique sequence of scalars $\left\{\alpha_{n}\right\}_{n=1}^{\infty}$ such that $x=\lim _{n \rightarrow \infty} \sum_{k=1}^{n} \alpha_{k} x_{k}$ for all $x \in X$. A sequence $\left\{x_{n}\right\}_{n=1}^{\infty}$ which is a Schauder basis of its closed span is called a basic sequence. A series $\sum_{n=1}^{\infty} x_{n}$ in a Banach space $X$ is unconditionally convergent if for every permutation $\sigma: \mathbb{N} \rightarrow \mathbb{N}$ the series $\sum_{n=1}^{\infty} x_{\sigma(n)}$ converges.

A space with a basis is always separable. The most of the natural separable spaces have bases, although Pel Enflo [27] was the first who found that there are separable Banach spaces without bases, looked inside $c_{0}$. The fact that a separable Banach space has a basis does provide some structural information about the space. It must be pointed out that finding a basis for a well know space is sometimes difficult. For instance, in the case of the classical separable sequence spaces $c_{0}$ and $\ell^{p}(1 \leq p<\infty)$, the sequence $\left\{e_{n}\right\}_{n=1}^{\infty}$ of unit coordinate vectors $e_{n}=\left(0,0, \ldots, 0,1_{(n-t h ~ p l a c e)}, 0, \ldots\right)$ is a basis. In the case of the space of convergent sequences $c$, if we denote $\mathbf{1}=(1,1, \ldots)$ then the sequence $\left(\mathbf{1}, e_{1}, e_{2}, \ldots\right)$ is a basis for $c$. In the case of $\mathcal{C}[0,1]$, the Schauder basis is a basis for this space, generally if $n \geq 1$ and $1 \leq i \leq 2^{n}$, then the sequence $f_{1}(t)=1, f_{2}(t)=$ $t, f_{3}(t)=2 t \chi_{[0,1 / 2]}(t)+2(2-t) \chi_{[1 / 2,1]}, \ldots, f_{2^{n}+i+1}(t)=f_{3}\left(2^{n} t+1-i\right)$ whenever $2^{n}+n-i \in[0,1]$ is a Schauder basis, see Figure 1.1. In the case of $L^{p}[0,1]$, where $1 \leq p<\infty$, the Haar system is a basis for this space, see 1.2. It is given 


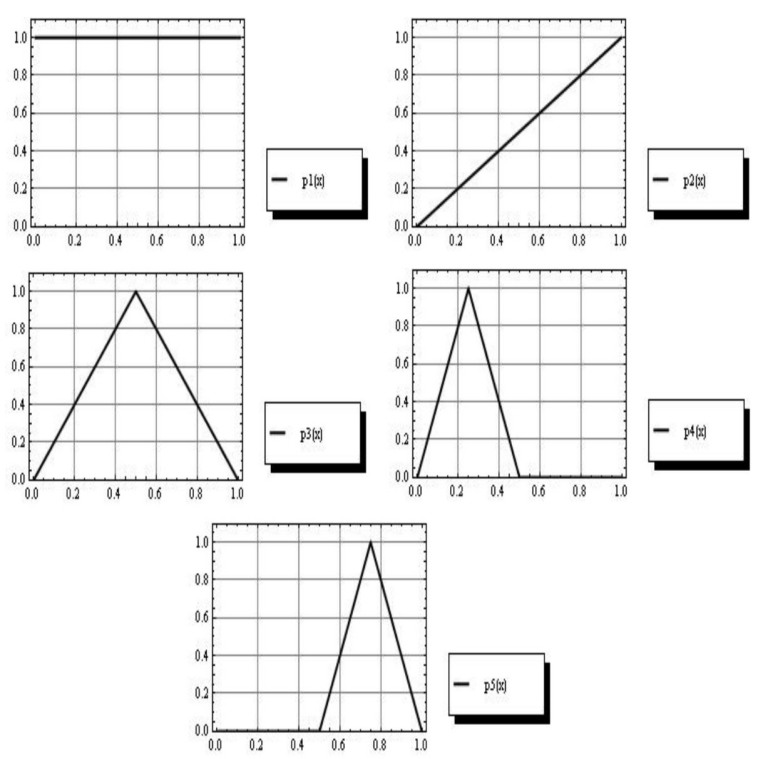

Figure 1.1: Schauder basis.

by

$$
\begin{gathered}
f_{0}(t)=1, \\
f_{2^{n}+i}(t)=\chi_{\left[(2 i-2) / 2^{n+1},(2 i-1) / 2^{n+1}\right]}(t)-\chi_{\left[(2 i-1) / 2^{n+1}, 2 i / 2^{n+1}\right]}(t),
\end{gathered}
$$

if $n \geq 1$ and $1 \leq i \leq 2^{n}$, and therefore if $1<p<\infty$ it is an unconditional basis. For the case of vector measures, there are examples such that the space of square integrable with respect to a particular vector measure is known, for instance, if $\mathbf{m}: \Sigma \longrightarrow L^{2}[0,1]$ and $\mathbf{m}(A)=\chi(A)$ then $L^{2}(\mathbf{m})=L^{4}[0,1]$. For this case the Haar system is a basis for $L^{2}(\mathbf{m})$. For the case of the spaces $L^{1}(\mathbf{m})$ it is also possible to find criteria for obtaining basic sequences, for instance Theorem 3 , [15]. In fact the Haar system is a basis for all $L^{p}(\mu), 1 \leq p<\infty$ (see Chapter $5,[24])$.

We use standard Banach function space notation. Let $\left\{x_{n}\right\}_{n}$ be a basic sequence. The symbol $\left[x_{n}\right]$ denote the smallest closed linear set spanned upon the elements $\left\{x_{n}\right\}_{n=1}^{\infty} \subset X$. The projections $P_{n}: X \rightarrow X$ defined by $P_{n}\left(\sum_{i=1}^{\infty} \alpha_{i} x_{i}\right)=\sum_{i=1}^{n} \alpha_{i} x_{i}$ are bounded operators and $\sup _{n}\left\|P_{n}\right\|<\infty$. The number $K=\sup _{n}\left\|P_{n}\right\|$ is called the basic constant of $\left\{x_{n}\right\}_{n=1}^{\infty}$. Equivalence of basic sequences (and in particular of bases) will become a powerful technique for 

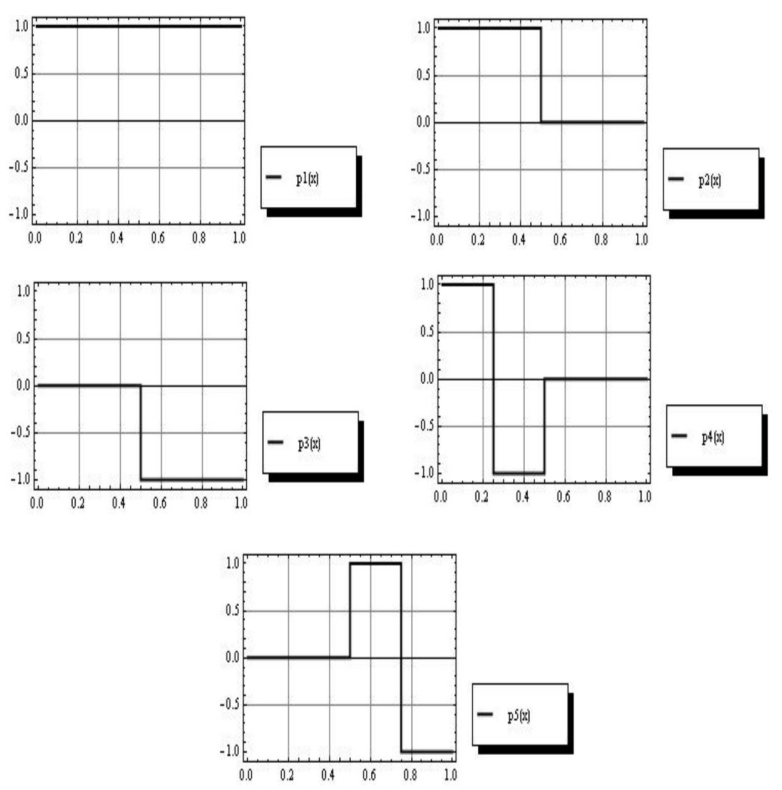

Figure 1.2: Haar basis.

studying the isomorphic structure of Banach spaces. In general, let $\left\{x_{n}\right\}_{n=1}^{\infty}$ and $\left\{y_{n}\right\}_{n=1}^{\infty}$ be two bases for the Banach spaces $X$ and $Y$ respectively. Then say that $\left\{x_{n}\right\}_{n=1}^{\infty}$ and $\left\{y_{n}\right\}_{n=1}^{\infty}$ are equivalents if and only if there exists a constant $C>0$ such that for all nonzero sequences of scalars $\left\{\alpha_{i}\right\}_{i=1}^{\infty}$,

$$
C^{-1}\left\|\sum_{i=1}^{\infty} \alpha_{i} y_{i}\right\| \leq\left\|\sum_{i=i}^{\infty} \alpha_{i} x_{i}\right\| \leq C\left\|\sum_{i=1}^{\infty} \alpha_{i} y_{i}\right\| .
$$

If the constant $C=1$ then the basic sequence $\left\{x_{n}\right\}_{n=1}^{\infty}$ and $\left\{y_{n}\right\}_{n=1}^{\infty}$ are said to be isometrically equivalent.

The following stability results dates back to 1940 [39].

Remark 1.2.1. If $\left\{x_{i}\right\}_{i=1}^{\infty}$ is a basic sequence of a Banach space $X$ and $\left\{y_{i}\right\}_{i=1}^{\infty}$ is another sequence in $\mathrm{X}$ so that $\left\|x_{n}-y_{n}\right\| \rightarrow 0$ then $\left\{y_{i}\right\}_{i=1}^{\infty}$ is a basic sequence.

Let $\left\{x_{i}\right\}_{i=1}^{\infty}$ be a basis sequence of a Banach space $X$ and take two sequences of positive integers $\left\{p_{i}\right\}_{i=1}^{\infty}$ and $\left\{q_{i}\right\}_{i=1}^{\infty}$ satisfying that $p_{i}<q_{i}<p_{i+1}$ for every $i \in \mathbb{N}$. A block basic sequence $\left\{y_{i}\right\}_{i=1}^{\infty}$ associated to $\left\{x_{i}\right\}_{i=1}^{\infty}$ is a sequence of 
vectors of $X$ defined as finite linear combinations as

$$
y_{i}=\sum_{k=p_{i}}^{q_{i}} \alpha_{i, k} x_{k}
$$

where $\alpha_{i, k}$ are real numbers (see also [24], Ch.V for the definition of block basic sequence and also for the standard definition of equivalence between basis). Let $S$ be a subspace of $X, S$ is called complemented in $X$ if and only if there exists a continuous projection from $S$ onto $X$.

Fundamental to the study of bases in a separable Hilbert space $H$ is the notion of a biorthogonal system. If $n, m$ are indexes of a set $I$, we write $\delta_{n, m}$ for the Kronecker's delta as usual. Two sequences $\left\{x_{n}\right\}_{n}$ and $\left\{y_{n}\right\}_{n}$ of elements from $H$ are said to be biorthogonal if $\left\langle x_{n}, y_{m}\right\rangle=\delta_{n, m}$.

Now, we consider the particular case when $X$ is a space of 2-integrable functions with respect to a vector measure $\mathbf{m}$. In this case, the question of how to recognize a basic sequence arises. The following remark provide a basic test for recognizing a basis in a subspace of $L^{2}(\mathbf{m})$, (see Theorem 1. Ch.V [24]).

Remark 1.2.2. Let $\left\{f_{n}\right\}_{n=1}^{\infty}$ be a sequence of non zero functions in $L^{2}(\mathbf{m})$, then in order $\left\{f_{n}\right\}_{n=1}^{\infty}$ to be a basic sequence, it is both necessary and sufficient that there exists a positive finite constant $K$ so that for any choice of scalars $\left\{\alpha_{i}\right\}_{i=1}^{\infty}$ and any integers $m<n$ we have

$$
\left\|\sum_{i=1}^{m} \alpha_{i} f_{i}\right\|_{L^{2}(\mathbf{m})} \leq K\left\|\sum_{i=1}^{n} \alpha_{i} f_{i}\right\|_{L^{2}(\mathbf{m})} .
$$

For instance, if $\mathbf{m}: \Sigma \rightarrow X$ is a positive vector measure, $\|f\|_{L^{2}(\mathbf{m})}=\left\|\int|f|^{2}\right\|_{X}^{1 / 2}$ for all $f \in L^{2}(\mathbf{m})$ (see [23]), and so the criterion above can be written as follows. For any finite sequences of scalars $\left\{\alpha_{i}\right\}_{i=1}^{\infty}$ and any integers $m<n$,

$$
\left\|\int\left(\sum_{i=1}^{m} \alpha_{i} f_{i}\right)^{2} d \mathbf{m}\right\|_{X} \leq K^{2}\left\|\int\left(\sum_{i=1}^{n} \alpha_{i} f_{i}\right)^{2} d \mathbf{m}\right\|_{X} .
$$

In Chapter 2 we will provide the adequate requirements on $\left\{f_{i}\right\}_{i=1}^{\infty}$ in order to obtain 1.7 that is equivalent to

$$
\left\|\sum_{i=1}^{m} \alpha_{i}^{2} \int f_{i}^{2} d \mathbf{m}\right\|_{X} \leq K^{2}\left\|\sum_{i=1}^{n} \alpha_{i}^{2} \int f_{i}^{2} d \mathbf{m}\right\|_{X} .
$$


Let $S$ be a subspace of $L^{2}(\mathbf{m})$ with a normalized basis $\left\{f_{n}\right\}_{n=1}^{\infty}$, if we perturb each element $f_{n} \in S$ by a sufficiently small vector we still get a basis and the perturbed basis is equivalent to the original one, see (Proposition 1.a.9, Vol I, [42]). The following remark show this.

Remark 1.2.3. Let $\left\{x_{n}\right\}_{n=1}^{\infty}$ be a normalized basis of a Banach space $X$ with a basis constant $K$. Let $\left\{y_{n}\right\}_{n=1}^{\infty}$ be a sequence of vectors in $X$ with $\sum_{n=1}^{\infty} \| x_{n}-$ $y_{n} \|<1 /(2 K)$. Then $\left\{y_{n}\right\}_{n=1}^{\infty}$ is a basis of $X$ which is equivalent to $\left\{x_{n}\right\}_{n=1}^{\infty}$.

In what follows we consider vector measures which take values in Hilbert spaces. We present a version of a principle for selecting basic sequences due to Bessaga and Pelczyński (Corollaries 1.2.4 and 2.4.3 below and see also [7]). We will consider first properties of the range of the vector measure and not of the space $L^{2}(\mathbf{m})$.

Corollary 1.2.4. Let $H$ be a Hilbert space and let $\mathbf{m}: \Sigma \longrightarrow H$ be a countably additive vector measure. Let $\left\{e_{n}\right\}_{n=1}^{\infty}$ be a basic sequence of $H$ and $\left\{e_{n}^{\prime}\right\}_{n}$ be a biorthogonal sequence to $\left\{e_{n}\right\}_{n}$. If the sequence $\left\{\int f_{n}^{2} d \mathbf{m}\right\}_{n=1}^{\infty}$ fulfills the condition

$$
\sum_{n=1}^{\infty}\left\|e_{n}-\int f_{n}^{2} d \mathbf{m}\right\|_{H}\left\|e_{n}^{\prime}\right\|_{H}=\delta<1,
$$

then $\left\{\int f_{n}^{2} d \mathbf{m}\right\}_{n=1}^{\infty}$ is a basic sequence which is equivalent to $e_{n}$.

Proof. For arbitrary integer $i, p, q$ such that $i<p<q$, we have

$$
\left|\alpha_{i}\right|=\left|e_{i}^{\prime}\left(\sum_{j=1}^{p} \alpha_{j} e_{j}\right)\right| \leq\left\|\sum_{j=1}^{p} \alpha_{j} e_{j}\right\|_{H}\left\|e_{i}^{\prime}\right\|_{H} .
$$

From the above expression, we obtain

$$
\begin{gathered}
\left\|\sum_{j=1}^{p} \alpha_{j}\left(\int f_{j}^{2} d \mathbf{m}\right)\right\|_{H} \leq\left\|\sum_{j=1}^{p} \alpha_{j} e_{j}\right\|_{H}+\left\|\sum_{j=1}^{p} \alpha_{j}\left(e_{j}-\int f_{j}^{2} d \mathbf{m}\right)\right\|_{H} \\
\leq\left\|\sum_{j=1}^{p} \alpha_{j} e_{j}\right\|_{H}+\sum_{j=1}^{p}\left|\alpha_{j}\right|\left\|e_{j}-\int f_{j}^{2} d \mathbf{m}\right\|_{H} \\
\leq\left\|\sum_{j=1}^{p} \alpha_{j} e_{j}\right\|_{H}+\sum_{i=1}^{\infty}\left|\alpha_{i}\right|\left\|e_{i}-\int f_{i}^{2} d \mathbf{m}\right\|_{H} \\
\leq\left\|\sum_{j=1}^{p} \alpha_{j} e_{j}\right\|_{H}+\sum_{i=1}^{\infty}\left\|\sum_{j=1}^{p} \alpha_{j} e_{j}\right\|_{H}\left\|e_{i}^{\prime}\right\|_{H}\left\|e_{i}-\int f_{i}^{2} d \mathbf{m}\right\|_{H}
\end{gathered}
$$




$$
\leq(1+\delta)\left\|\sum_{j=1}^{p} \alpha_{j} e_{j}\right\|_{H}
$$

and using the same computations that above, we obtain

$$
\begin{aligned}
\left\|\sum_{j=1}^{q} \alpha_{j}\left(\int f_{j}^{2} d \mathbf{m}\right)\right\|_{H} & \geq\left|\left\|\sum_{j=1}^{q} \alpha_{j} e_{j}\right\|_{H}-\left\|\sum_{j=1}^{q} \alpha_{j}\left(e_{j}-\int f_{j}^{2} d \mathbf{m}\right)\right\|_{H}\right| \\
& \geq(1-\delta)\left\|\sum_{j=1}^{q} \alpha_{j} e_{j}\right\|_{H} .
\end{aligned}
$$

Hence

$$
\left\|\sum_{j=1}^{p} \alpha_{j}\left(\int f_{j}^{2} d \mathbf{m}\right)\right\|_{H} \leq K \frac{1+\delta}{1-\delta}\left\|\sum_{j=1}^{q} \alpha_{j}\left(\int f_{j}^{2} d \mathbf{m}\right)\right\|_{H} .
$$

Remark 1.2.5. In general, if we take $\left\{x_{n}\right\}_{n=1}^{\infty}$ a weakly null, normalized sequence in a Banach space $X$ then $\left\{x_{n}\right\}_{n=1}^{\infty}$ admits a subsequence that is a basic sequence, (see Bessaga-Pelczynski Selection Principle theorem [24]).

Example 1.2.6. Consider the Lebesgue measure space $([0, \infty), \Sigma, d x)$ and define the positive vector measure $\nu: \Sigma \rightarrow c_{0}$ as

$$
\nu(A)=\sum_{n=0}^{\infty}\left(\int_{A \cap[n, n+1]}(x-n)^{n} d x\right) e_{n} .
$$

It is clearly countably additive and then the corresponding space $L^{2}(\nu)$ is welldefined. If $f \in L^{1}(\nu)$ then $\int f d \nu=\left\{\int_{[n, n+1]} f(x)(x-n)^{n} d x\right\}_{n=1}^{\infty} \in c_{0}$. Consider now the sequence of functions $\left\{f_{k}\right\}_{k=0}^{\infty}=\left\{e^{-k(x-k) / 2} \chi_{[k, \infty)}(x)\right\}_{k=0}^{\infty}$, then $f_{k}^{2}(x)=e^{-k(x-k)} \chi_{[k, \infty)}(x) \in L^{1}(\nu)$ and so $f_{k} \in L^{2}(\nu)$.

$\left\|f_{k}\right\|_{L^{2}(\nu)}^{2}=\left\|\int_{[0, \infty)} f_{k}^{2} d \nu\right\|_{c_{0}}=\left\|\left\{\int_{[n, n+1]} e^{-k(x-k)} \chi_{[k, \infty)}(x)(x-k)^{n} d x\right\}_{n=0}^{\infty}\right\|_{c_{0}}$

and note that for each $k$ we find a constant $M_{k}$ as follows,

$$
\begin{gathered}
\sup _{n}\left\{\int_{[n, n+1]} e^{-k(x-k)} \chi_{[k, \infty)}(x)(x-n)^{n} d x\right\}_{n=0}^{\infty} \\
=\int_{[k, k+1]} e^{-k(x-k)}(x-k)^{k} d x=M_{k}<\infty .
\end{gathered}
$$


Thus is easy to find a normalized sequence for applying the Remark 1.2.5, since that for all $n \in \mathbb{N}$

$$
\begin{gathered}
\lim _{k \rightarrow \infty}\left\langle\int_{[0, \infty)} \frac{f_{k}^{2}}{M_{k}} d \nu, e_{n}\right\rangle=\lim _{k \rightarrow \infty} \int_{[n, n+1]} \frac{f_{k}^{2}}{M_{k}}(x-n)^{n} d x \\
=\lim _{k \rightarrow \infty} \int_{[n, n+1]} \frac{e^{-k(x-k)}}{M_{k}}(x-n)^{n} \chi_{[k, \infty]}(x) d x=0 .
\end{gathered}
$$

Therefore $\left\{f_{k}\right\}_{k=1}^{\infty}$ admits a subsequence that is a basic sequence.

\subsection{Kadec-Pelczyński decomposition}

The aim of this section is to give a canonical procedure for obtaining disjoint sequences in the space $L^{2}(\mathbf{m})$. This will be the first step for finding $\mathbf{m}-$ orthogonal sequences (see Definition 2.3.1), and providing the corresponding existence theorems. In what follows a well known result of Kadec and Pelczyński (see for instance [38]) will be applied to the context of sequences of functions on spaces of integrable functions with respect to a vector measure. Through this section we will consider a positive vector measure $\mathbf{m}$.

Let $H$ be a Hilbert space and let us consider a positive countably additive vector measure $\mathbf{m}: \Sigma \rightarrow H$. We suppose that $\left\|\chi_{\Omega}\right\|_{L^{2}(\mathbf{m})}=\left\|\chi_{\Omega}\right\|_{L^{1}(\mathbf{m})}^{1 / 2}=$ $\|\mathbf{m}\|(\Omega)=1$ and $\left\{f_{n}\right\}_{n=1}^{\infty} \in L^{2}(\mathbf{m})$. We define the subsets of $\Omega$

$$
\sigma(f, \varepsilon)=\left\{t \in \Omega:|f(t)| \geq \varepsilon\|f\|_{L^{2}(\mathbf{m})}\right\}
$$

and the subsets of $L^{2}(\mathbf{m})$

$$
M_{L^{2}(\mathbf{m})}(\varepsilon)=\left\{f \in L^{2}(\mathbf{m}):\|\mathbf{m}\|(\sigma(f, \varepsilon)) \geq \varepsilon\right\} .
$$

By normalizing if necessary, we assume that $\left\|f_{n}\right\|_{L^{2}(\mathbf{m})}=1$ for all $n \in \mathbb{N}$.

Remark 1.3.1. Note that the classes $M_{L^{2}(\mathbf{m})}(\varepsilon)$ have the following properties:

(1) If $\varepsilon_{1}<\varepsilon_{2}$, then $M_{L^{2}(\mathbf{m})}\left(\varepsilon_{1}\right) \supset M_{L^{2}(\mathbf{m})}\left(\varepsilon_{2}\right)$.

(2) $\bigcup_{\varepsilon>0} M_{L^{2}(\mathbf{m})}(\varepsilon)=L^{2}(\mathbf{m})$. 
(3) If $f \neq 0$ does not belong to $M_{L^{2}(\mathbf{m})}(\varepsilon)$, then there exists a set $A$ such that $\|\mathbf{m}\|(A)<\varepsilon$ and

$$
\left\|\int_{A}\left|\frac{f(t)}{\|f\|_{L^{2}(\mathbf{m})}}\right|^{2} d \mathbf{m}\right\|_{H} \geq 1-\varepsilon^{2} .
$$

The first property is obvious. To prove the second, we suppose that there exists a square $\mathbf{m}$-integrable function $g$ so that it is not in $\bigcup_{\varepsilon>0} M_{L^{2}(\mathbf{m})}(\varepsilon)$ for all $\varepsilon>0$, in particular $g \neq 0$, that is, $\|\mathbf{m}\|($ Supp $g)>0$. Since Supp $g=$ $\cup_{n \geq 1} \sigma\left(g, \frac{\varepsilon}{2^{n}}\right)$ for every $\varepsilon>0$, then

$$
\|\mathbf{m}\|(\text { Supp } g) \leq \sum_{n \geq 1}\|\mathbf{m}\|\left(\sigma\left(g, \frac{\varepsilon}{2^{n}}\right)\right) \leq \sum_{n \geq 1} \frac{\varepsilon}{2^{n}}=\varepsilon .
$$

So $\|\mathbf{m}\|($ Supp $g)=0$ which is a contradiction. For proving the third we denote by $A$ the set $\sigma(f, \varepsilon)$. Then

$$
\begin{aligned}
& 1=\left\|\int_{\Omega}\left|\frac{f(t)}{\|f\|_{L^{2}(\mathbf{m})}}\right|^{2} d \mathbf{m}\right\|_{H} \leq\left\|\int_{A}\left|\frac{f(t)}{\|f\|_{L^{2}(\mathbf{m})}}\right|^{2} d \mathbf{m}\right\|_{H}+\varepsilon^{2}\|\mathbf{m}(\Omega / A)\|_{H} \\
& \leq\left\|\int_{A}\left|\frac{f(t)}{\|f\|_{L^{2}(\mathbf{m})}}\right|^{2} d \mathbf{m}\right\|_{H}+\varepsilon^{2}\|\mathbf{m}\|(\Omega / A) \leq\left\|\int_{A}\left|\frac{f(t)}{\|f\|_{L^{2}(\mathbf{m})}}\right|^{2} d \mathbf{m}\right\|_{H}+\varepsilon^{2} .
\end{aligned}
$$

This finishes the proof of (3).

Lemma 1.3.2. Let $(\Omega, \Sigma, \mu)$ be a measure space and let $X(\mu)$ be an order continuous Banach function space, then for all $f \in X(\mu)$

$$
\lim _{\mu(A) \rightarrow 0}\left\|f \chi_{A}\right\|_{X(\mu)}=0 .
$$

Proof. We suppose that $\lim _{\mu(A) \rightarrow 0}\left\|f \chi_{A}\right\|_{X(\mu)} \neq 0$. Then there exists a sequence of subsets $A_{1}, A_{2}, \ldots, A_{n}, \ldots$ into $\Sigma$ such that $\lim _{n \rightarrow \infty} \mu\left(A_{n}\right)=0$ and $\left\|f \chi_{A_{n}}\right\|_{X(\mu)}>\delta>0$ for all $n \in \mathbb{N}$. We take a subsequence $\tilde{A}_{1}, \ldots, \tilde{A}_{n}, \ldots$ of $\left(A_{n}\right)_{n}$ such that $0<\mu\left(\tilde{A}_{i}\right) \leq \frac{1}{2^{i}}$, and we define the following collection of sets

$$
B_{1}=\bigcup_{k=1}^{\infty} \tilde{A}_{k}, \quad B_{2}=\bigcup_{k=2}^{\infty} \tilde{A}_{k}, \quad \ldots, \quad B_{n}=\bigcup_{k=n}^{\infty} \tilde{A}_{k}, \quad \ldots
$$

It follows that $\mu\left(B_{n}\right) \leq \sum_{k=n}^{\infty} \mu\left(\tilde{A}_{k}\right) \leq \sum_{k=n}^{\infty} 1 / 2^{k} \leq 1 / 2^{n-1}$. On the other hand $\mu\left(B_{n}\right)=\left\|\chi_{B_{n}}\right\|_{X(\mu)}$ and therefore $\left\|\chi_{B_{n}}\right\|_{X(\mu)}$ converges to $0 \mu$-a.e. So there exists a subsequence $\chi_{B_{n_{j}}}$ of $\chi_{B_{n}}$ that converges pointwise to 0 and 
$f \chi_{B_{n_{j}}}$ converges to 0 . From the order continuity we deduce that $\left\|f \chi_{B_{n_{j}}}\right\|_{L^{1}(\mu)}$ converges to $0 \mu$-a.e. For every $n_{j}$, since $\tilde{A}_{n_{j}} \subset B_{n_{j}}$, we have that $\delta<$ $\left\|\chi_{\tilde{A}_{n_{j}}} f\right\|_{X(\mu)} \leq\left\|\chi_{B_{n_{j}}} f\right\|_{X(\mu)}$. This gives a contradiction and proves the lemma.

The following result shows two mutually excluding possibilities for a sequence $\left\{f_{n}\right\}_{n}$ of functions in $L^{2}(\mathbf{m})$. On one hand, when $\left\{f_{n}\right\}_{n}$ is included in the set $M_{L^{2}(\mathbf{m})}(\varepsilon)$ for some $\varepsilon>0$, the norms $\|\cdot\|_{L^{2}(\mathbf{m})}$ and $\|\cdot\|_{L^{1}(\mu)}$ are equivalent, where $\mu$ is a Rybakov control measure for $\mathbf{m}$. On the other hand, when $\left\{f_{n}\right\}_{n} \nsubseteq M_{L^{2}(\mathbf{m})}(\varepsilon)$ for every $\varepsilon>0$, we can built another sequence $\left\{h_{k}\right\}_{k}$ of disjoint functions of $L^{2}(\mathbf{m})$, in such a way that $\left\{f_{n}\right\}_{n}$ and $\left\{h_{k}\right\}_{k}$ are equivalent (see Chapter 1.9. [32]). This procedure gives us a tool for building disjoint sequences in subspaces of $L^{2}(\mathbf{m})$ that in fact are unconditional basic sequences. The order continuity of the space is the key point for the construction.

Theorem 1.3.3. Let $\mu=\left|\mathbf{m}_{x_{0}^{\prime}}\right|$ be a Rybakov measure for a vector measure $\mathbf{m}$ and let $(\Omega, \Sigma, \mu)$ be a probability measure space. Let $\left\{f_{n}\right\}_{n}$ be a sequence of functions into $L^{2}(\mathbf{m})$.

(1) If $\left\{f_{n}\right\}_{n=1}^{\infty} \subset M_{L^{2}(\mathbf{m})}(\varepsilon)$ for some $\varepsilon>0$ then $\left\{f_{n}\right\}_{n=1}^{\infty}$ converges to zero in $L^{2}(\mathbf{m})$ if and only if $\left\{f_{n}\right\}_{n=1}^{\infty}$ converges to zero in $L^{1}(\mu)$.

(2) If $\left\{f_{n}\right\}_{n=1}^{\infty} \nsubseteq M_{L^{2}(\mathbf{m})}(\varepsilon)$ for all $\varepsilon>0$ then there exists a subsequence $\left\{n_{k}\right\}_{k=1}^{\infty}$ and a disjointly supported functions $\left\{h_{k}\right\}_{k=1}^{\infty} \subset L^{2}(\mathbf{m})$ such that $\left|h_{k}\right| \leq\left|f_{n_{k}}\right|$ for all $k$ and $\left\{h_{k}\right\}_{k=1}^{\infty}$ and $\left\{f_{n_{k}}\right\}_{k=1}^{\infty}$ are equivalent unconditional basic sequences that satisfy $\lim _{k \rightarrow \infty}\left\|f_{n_{k}}-h_{k}\right\|_{L^{2}(\mathbf{m})}=0$.

Proof. It is well known that $L^{2}(\mathbf{m})$ is continuously embedded into $L^{1}(\mu)$ and it is an order continuous Banach lattice with weak unit. There are two excluding cases.

(1) We suppose that $\left\{f_{n}\right\}_{n} \subset M_{L^{2}(\mathbf{m})}(\varepsilon)$ for some $\varepsilon>0$ then

$$
\begin{gathered}
\left\|f_{n}\right\|_{L^{2}(\mathbf{m})} \geq\left\|f_{n}\right\|_{L^{1}(\mu)}=\int_{\Omega}\left|f_{n}(t)\right| d \mu \geq \int_{\sigma\left(f_{n}, \varepsilon\right)}\left|f_{n}(t)\right| d \mu \\
\geq \varepsilon\left\|f_{n}\right\|_{L^{2}(\mathbf{m})} \mu\left(\sigma\left(f_{n}, \varepsilon\right)\right) .
\end{gathered}
$$

The direct implication is obtained from the inclusion $L^{2}(\mathbf{m}) \hookrightarrow L^{1}(\mu)$. Conversely, we suppose that $\mu\left(\sigma\left(f_{n}, \varepsilon\right)\right)$ converges to 0 . Since $\mu$ is a Rybakov measure and thus it is a control measure $\|\mathbf{m}\|\left(\sigma\left(f_{n}, \varepsilon\right)\right)$ converges 
to 0 , but it gives a contradiction because $M_{L^{2}(\mathbf{m})}(\varepsilon)=\left\{f \in L^{2}(\mathbf{m})\right.$ : $\|\mathbf{m}\|(\sigma(f, \varepsilon)) \geq \varepsilon\}$. Therefore, $\left(f_{n}\right)$ converges to zero in $L^{2}(\mathbf{m})$ if and only if $\left(f_{n}\right)$ converges to zero in $L^{1}(\mu)$.

(2) If the above supposition does not hold, then $\left(f_{n}\right)_{n} \nsubseteq M_{L^{2}(\mathbf{m})}(\varepsilon)$ for all $\varepsilon$. In order to simplify the notation we consider $\left\|f_{n}\right\|_{L^{2}(\mathbf{m})}=1$. Thus, there exists an index $n_{1} \in \mathbb{N}$ such that $f_{n_{1}}$ is not in $M_{L^{2}(\mathbf{m})}(\varepsilon)$ where $j_{1}=2$. We take $\varepsilon=4^{-j_{1}}$. Then $\|\mathbf{m}\|\left(\sigma\left(f_{n_{1}}, 4^{-j_{1}}\right)\right)<4^{-j_{1}}$ and

$$
\begin{gathered}
\left\|\chi_{\sigma\left(f_{n_{1}}, 4^{-j_{1}}\right)^{c}} f_{n_{1}}\right\|_{L^{2}(\mathbf{m})}=\left\|\int\left|\chi_{\sigma\left(f_{n_{1}}, 4^{-j_{1}}\right)^{c}} f_{n_{1}}\right|^{2} d \mathbf{m}\right\|_{H}^{1 / 2} \\
=\left\|\int_{\chi_{\sigma\left(f_{n_{1}}, 4^{-j_{1}}\right)^{c}}}\left|f_{n_{1}}\right|^{2} d \mathbf{m}\right\|_{H}^{1 / 2} \leq 4^{-j_{1}}\left\|\mathbf{m}\left(\sigma\left(f_{n_{1}}, 4^{-j_{1}}\right)^{c}\right)\right\|_{H}^{1 / 2} \\
\leq 4^{-j_{1}}\|\mathbf{m}\|(\Omega)^{1 / 2}=4^{-j_{1}} .
\end{gathered}
$$

Now we apply 1.3 .2 , so there exists $\delta_{1}>0$ such that for all $A \in \Sigma$ with $\|\mathbf{m}\|(A)<\delta_{1}$ it follows $\left\|\chi_{A} f_{n_{1}}\right\|<4^{-\left(j_{1}+1\right)}$. We take $j_{2}>j_{1}$ such that $4^{-j_{2}}<\delta_{1}$. By the same argument, there exists $n_{2}>n_{1}$ such that $f_{n_{2}}$ is not in $M_{L^{2}(\mathbf{m})}\left(4^{-j_{2}}\right)$, thus $\|\mathbf{m}\|\left(\sigma\left(f_{n_{2}}, 4^{-j_{2}}\right)\right)<4^{-j_{2}}<\delta_{1}$

$$
\begin{gathered}
\left\|\chi_{\sigma\left(f_{n_{2}}, 4^{-j_{2}}\right)^{c}} f_{n_{1}}\right\| \leq 4^{-\left(j_{1}+1\right)}, \\
\left\|\chi_{\sigma\left(f_{n_{2}}, 4^{-j_{2}}\right)^{c}} f_{n_{2}}\right\| \leq\left\|4^{-j_{2}} \chi_{\sigma\left(f_{n_{2}}, 4^{-j_{2}}\right)^{c}}\right\| \leq 4^{-j_{2}} .
\end{gathered}
$$

We take $\varepsilon=4^{-\left(j_{2}+1\right)}$. Again, we apply Lemma 1.3.2 and there exists $\delta_{2}>$ 0 such that for all $A \in \Sigma$ with $\|\mathbf{m}\|(A)<\delta_{2}$ it follows $\left\|\chi_{A} f_{n_{1}}\right\|,\left\|\chi_{A} f_{n_{2}}\right\|<$ $4^{-\left(j_{2}+1\right)}$. Let $j_{3}>j_{2}$ be an integer satisfying that $4^{-j_{3}}<\delta_{2}$. Again there exists a integer $n_{3}>n_{2}>n_{1}$ such that $f_{n_{3}}$ is not in $M_{L^{2}(\mathbf{m})}\left(4^{-j_{3}}\right)$, as in the above case we have $\|\mathbf{m}\|\left(\sigma\left(f_{n_{3}}, 4^{-j_{3}}\right)\right)<4^{-j_{3}}<\delta_{2}$, and therefore

$$
\begin{gathered}
\left\|\chi_{\sigma\left(f_{n_{3}}, 4^{\left.-j_{3}\right)}\right.} f_{n_{1}}\right\|, \quad\left\|\chi_{\sigma\left(f_{n_{3}}, 4^{-j_{3}}\right)} f_{n_{2}}\right\| \leq 4^{-\left(j_{2}+1\right)}, \\
\left\|\chi_{\sigma\left(f_{n_{3}}, 4^{-j_{3}}\right)^{c}} f_{n_{3}}\right\| \leq 4^{-j_{3}} .
\end{gathered}
$$

In the same way, it is possible to find two subsequences $\left\{f_{n_{k}}\right\}_{k=1}^{\infty}$ and $\sigma\left(f_{n_{k}}, 4^{-j_{k}}\right)_{k}$ that satisfy the following inequalities:

$$
\begin{gathered}
\|\mathbf{m}\|\left(\sigma\left(f_{n_{k}}, 4^{-j_{k}}\right)\right)<4^{-j_{k}}, \\
\left\|\chi_{\sigma\left(f_{n_{k}}, 4^{-j_{k}}\right)^{c}} f_{n_{k}}\right\| \leq 4^{-j_{k}},
\end{gathered}
$$




$$
\left\|\chi_{\sigma\left(f_{n_{k}}, 4^{\left.-j_{k}\right)}\right.} f_{n_{i}}\right\| \leq 4^{-\left(j_{k-1}+1\right)}, \quad i=1, \ldots, k-1 .
$$

Now, we define the following disjoint sequence of sets:

$$
\begin{gathered}
\varphi_{k}=\sigma\left(f_{n_{k}}, 4^{-j_{k}}\right) \backslash \bigcup_{i=k+1}^{\infty} \sigma\left(f_{n_{i}}, 4^{-j_{i}}\right) . \\
\varphi_{k}^{c}=\sigma\left(f_{n_{k}}, 4^{-j_{k}}\right)^{c} \bigcup\left(\bigcup_{i=k+1}^{\infty} \sigma\left(f_{n_{i}}, 4^{-j_{i}}\right)\right) .
\end{gathered}
$$

Thus $\varphi_{k} \cap \varphi_{l}=\emptyset$ for $k \neq l$. This allows to construct the sequence of disjoint functions $h_{k}=\chi_{\varphi_{k}} f_{n_{k}}$. Due to the lattice properties of $L^{2}(\mathbf{m})$ and Remark 1.2.2 we obtain that the sequence $\left\{h_{k}\right\}_{k=1}^{\infty}$ is a basic sequence. On the other hand we check that $\lim _{k \rightarrow \infty}\left\|f_{n_{k}}-h_{k}\right\|=0$. Indeed

$$
\begin{gathered}
\left\|f_{n_{k}}-h_{k}\right\|=\left\|\chi_{\varphi_{k}^{c} f_{n_{k}}}\right\| \leq\left\|\chi_{\sigma\left(f_{n_{k}}, 4^{\left.-j_{k}\right)^{c}}\right.} f_{n_{k}}\right\|+\left\|\chi_{\bigcup_{i=k+1}^{\infty} \sigma\left(f_{n_{i}}, 4^{\left.-j_{i}\right)}\right.} f_{n_{k}}\right\| \\
\leq 4^{-j_{k}}+\sum_{i=k+1}^{\infty}\left\|\chi_{\sigma\left(f_{n_{i}}, 4^{\left.-j_{i}\right)}\right.} f_{n_{k}}\right\| \leq 4^{-j_{k}}+\sum_{i=k+1}^{\infty} 4^{-\left(j_{i-1}+1\right)} \\
\leq 4^{-j_{k}}+\frac{4^{-\left(j_{k}+1\right)}}{1-1 / 4}=4^{-j_{k}}+\frac{1}{3} 4^{-j_{k}}=\frac{1}{3} 4^{-\left(j_{k}-1\right)} .
\end{gathered}
$$

So if we apply Remark 1.2.1 and 1.5, we obtain that $\left\{f_{n_{k}}\right\}_{k=1}^{\infty}$ and $\left\{h_{k}\right\}_{k=1}^{\infty}$ are equivalent unconditional basic sequences. 


\section{Chapter 2}

\section{m-Orthogonal sequences with respect to a vector measure}

\section{$2.1 \mathrm{~m}$-Orthogonal sequences of functions with respect to a vector measure}

Given a measure space $(\Omega, \Sigma, \mu)$ where $\mu$ is an scalar measure, a sequence $\left\{f_{n}\right\}_{n=1}^{\infty}$ in $L^{2}(\mu)$ integrable functions is said to be $\mu$-orthogonal, if $\int f_{n} f_{m} d \mu=$ 0 for $m \neq n$ holds and none of the functions $f_{n}$ vanishes almost everywhere. In this chapter we present reasonable extensions of this notion when the measure involved is a positive vector measure $\mathbf{m}: \Sigma \rightarrow X$ where $X$ is a Banach lattice. Our aim is to show that these definitions lead us to different geometrical properties of the subspaces generated by the sequences of functions. Actually, the notion of orthogonality of two functions with respect to a vector measure can be broached under different perspectives.

We recall that if $f, g \in L^{2}(\mathbf{m})$ then $f g \in L^{1}(\mathbf{m})$ (see [60]); so the integral $\int f g d \mathbf{m}$ is well-defined. The representation theorem for 2-convex order continuous Banach lattices with a weak unit establishes that such an space can be al- 
ways identified (isomorphically and in order) with a space $L^{2}(\mathbf{m})$ of 2-integrable functions with respect to a positive Banach lattice valued vector measure $\mathbf{m}$ on a $\sigma$-algebra (see [28, Proposition 2.4] or [49, Proposition 3.9]). Although these spaces are not in general Hilbert spaces, the integration structure of the spaces $L^{2}(\mathbf{m})$ provides several extensions of the notion of orthogonality. Some theoretical results and applications have been already obtained in this setting. The notion of (weak, strong) orthogonality with respect to a vector measure $\mathbf{m}$ in spaces $L^{2}(\mathbf{m})$ of 2-integrable functions has been defined an studied in the last ten years, see for instance $([35,36])$. In this memoir we consider the three notions of orthogonality with respect to a vector measure, the weak orthogonality, the orthogonality itself and the strongly version of it that we will formalize in the following sections.

\subsection{Weak m-orthogonal sequences}

Definition 2.2.1. A sequence of functions $\left\{f_{n}\right\}_{n=1}^{\infty}$ in $L^{2}(\mathbf{m})$ is weak $\mathbf{m}$ orthogonal if there is an element $x^{\prime} \in\left(X^{\prime}\right)_{\mathbf{m}}^{+}$such that $\int f_{i}^{2} d\left\langle\mathbf{m}, x^{\prime}\right\rangle>0$ for all $i \in \mathbb{N}$, and for all $i \neq j$

$$
\left\langle\int f_{i} f_{j} d \mathbf{m}, x^{\prime}\right\rangle=\int f_{i} f_{j} d\left\langle\mathbf{m}, x^{\prime}\right\rangle=0 .
$$

For such a sequence we also say that it is orthogonal with respect to $\left\langle\mathbf{m}, x^{\prime}\right\rangle$ when an explicit reference to the scalar measure $\left\langle\mathbf{m}, x^{\prime}\right\rangle$ is convenient.

Although for many purposes it is not necessary, we will assume that $\mathbf{m}$ is a positive vector measure. It is easy to find examples of sequences that satisfy this property.

Example 2.2.2. (1) Consider the Lebesgue measure space $([0,1], \Sigma, d x)$. We can define the positive vector measure $\nu: \Sigma \rightarrow c_{0}$ as $\nu(A)=\left\{\int_{A} x^{n} d x\right\}_{n=1}^{\infty}$. It is clearly countably additive and then the corresponding space $L^{2}(\nu)$ is well-defined. Consider now the sequence of functions

$$
f_{i}(x)=\sqrt{2} e^{-x / 2} \sin (2 \pi i x) \quad i \in \mathbb{N} .
$$

Note that $f_{i}^{2} \leq 2 \in L^{1}(\nu)$ and so $f_{i} \in L^{2}(\nu)$. Take the sequence $x_{0}^{\prime}:=$ $\left\{\frac{1}{n ! e}\right\}_{n=0}^{\infty} \in \ell^{1}=\left(c_{0}\right)^{\prime}$. A direct calculation shows that $\int f_{i} f_{j} d\left\langle\nu, x_{0}^{\prime}\right\rangle=$ $\delta_{i, j} \frac{1}{e}$. 
(2) Let $\gamma:=\operatorname{arcsinh}\left(\frac{1}{2}\right)$. Take the Lebesgue measure space $([-\gamma, \gamma], \Sigma, d x)$. The positive countably additive vector measure $\nu(A)=\left\{\int_{A} x^{2 n} d x\right\}_{n=0}^{\infty} \in$ $c_{0}$ is then well defined. Note that if $f \in L^{1}(\nu)$ then

$$
\int f d \nu=\left\{\int_{-\gamma}^{\gamma} f(x) x^{2 n} d x\right\}_{n} \in c_{0} .
$$

Consider the sequence $f_{m}(x)=\cos (2 m \pi \sinh (x))$. Note that $f_{m}^{2} \leq$ $1 \in L^{1}(\nu)$ and so $f_{m} \in L^{2}(\nu)$. Take the norm one sequence $x_{0}^{\prime}:=$ $\left\{\frac{1}{\cosh (1)(2 n) !}\right\}_{n=0}^{\infty} \in \ell^{1}=\left(c_{0}^{\prime}\right)$. A direct calculation shows that

$$
\int f_{n} f_{m} d\left\langle\nu, x_{0}^{\prime}\right\rangle=\frac{1}{2 \cosh (1)} \delta_{m, n} .
$$

In what follows we provide a characterization of the situation given in the example above, i.e. when we can find an element $x^{\prime}$ such that the sequence $\left\{f_{n}\right\}_{n}$ is orthogonal in the space $L^{2}\left(\left\langle\mathbf{m}, x^{\prime}\right\rangle\right)$. Let us introduce first some notation and remarks. A family $\Phi$ of $\mathbb{R}$-valued defined on a non-empty set $\Psi$ is called concave if, for every finite set $\left\{\phi_{1}, \ldots, \phi_{n}\right\} \subseteq \Phi$ with $n \in \mathbb{N}$ and non negative scalars $\gamma_{1}, \ldots, \gamma_{n}$ satisfying $\sum_{j=1}^{n} \gamma_{j}=1$, there exists $\phi \in \Phi$ such that $\sum_{j=1}^{n} \gamma_{j} \phi_{j} \leq \phi$ pointwise on $\Psi$. The following result is known as Ky Fan's Lemma. Let $\Psi$ be a compact convex subset of a Hausdorff topological vector space and let $\Phi$ be a concave family of lower semi-continuous, convex, $\mathbb{R}$-valued functions defined on $\Psi$. Let $\gamma \in \mathbb{R}$. Suppose that for every $\phi \in \Phi$ there exists $x_{\phi} \in \Psi$ such that $\phi\left(x_{\phi}\right) \leq \gamma$. Then there exists $x \in \Psi$ such that $\phi(x) \leq \gamma$ for all $\phi \in \Phi$.

Let $\mathbf{m}: \Sigma \rightarrow X$ be a positive vector measure and take a sequence $S=\left\{f_{i}\right\}_{i=1}^{\infty} \subseteq$ $L^{2}(\mathbf{m})$ and a sequence of positive real numbers $\Delta=\left\{\varepsilon_{i}\right\}_{i=1}^{\infty}$. Then we write $B_{S, \Delta}$ for the convex weak* compact subset

$$
B_{S, \Delta}:=B_{X^{\prime}} \cap\left(X^{\prime}\right)_{\mathbf{m}}^{+} \cap\left\{x^{\prime}:\left\langle\int f_{i}^{2} d \mathbf{m}, x^{\prime}\right\rangle \leq \varepsilon_{i}, \quad \text { for all } i \in \mathbb{N}\right\} .
$$

Let us define the following continuous seminorm on $L^{1}(\mathbf{m})$.

$$
\|f\|_{B_{S, \Delta}}:=\sup _{x^{\prime} \in B_{S, \Delta}}\left(\int|f| d\left\langle\mathbf{m}, x^{\prime}\right\rangle\right) .
$$

For every $i, j \in \mathbb{N}, i \neq j$, let us write

$$
\varphi_{i, j, \theta}(w):=\left(f_{i}(w)+\theta f_{j}(w)\right)^{2}, \quad w \in \Omega,
$$

where $\theta \in\{-1,1\}$. Notice that $0 \leq \varphi_{i, j, \theta} \in L^{1}(\mathbf{m})$. 
For instance, in Example 2.2.2(1) the sequence $\Delta$ is $\Delta=\left\{\varepsilon_{i}\right\}_{i=0}^{\infty}=\left\{\frac{1}{e}\right\}_{i=0}^{\infty}$, and so $B_{S, \Delta} \supset \frac{1}{e} B_{\ell^{1}} \cap\left(\ell^{1}\right)_{\nu}^{+}$. Thus, $\|\cdot\|_{B_{S, \Delta}}$ is equivalent to the norm of $L^{1}(\nu)$.

In the following result the scalar product notation $\left\langle\left(\gamma_{k}\right),\left(\delta_{k}\right)\right\rangle:=\sum_{k=1}^{n} \gamma_{k} \delta_{k}$ for finite sequences $\left\{\gamma_{k}\right\}_{k=1}^{n}$ and $\left\{\delta_{k}\right\}_{k=1}^{n}$ is used.

Theorem 2.2.3. Let $\mathbf{m}: \Sigma \rightarrow X$ be a positive vector measure. Consider a sequence $S=\left\{f_{i}\right\}_{i} \subseteq L^{2}(\mathbf{m})$ and a sequence of positive real numbers $\Delta=\left\{\varepsilon_{i}\right\}_{i}$. The following statements are equivalent.

(1) For every finite sequence of non negative real numbers $\left\{\gamma_{k}\right\}_{k}$ such that $\sum_{k} \gamma_{k}=1$, indexes $i_{k}, j_{k} \in \mathbb{N}, i_{k} \neq j_{k}$, and $\theta_{k} \in\{-1,1\}$,

$$
\left\langle\left(\gamma_{k}\right),\left(\varepsilon_{i_{k}}+\varepsilon_{j_{k}}\right)\right\rangle \leq\left\|\left\langle\left(\gamma_{k}\right),\left(\varphi_{i_{k}, j_{k}, \theta_{k}}\right)\right\rangle\right\|_{B_{S, \Delta}} .
$$

(2) There is an element $0 \leq x_{0}^{\prime} \in B_{X^{\prime}}$ such that $S$ is weak $\mathbf{m}$-orthogonal with respect to $\left\langle\mathbf{m}, x_{0}^{\prime}\right\rangle$ and $\int f_{i}^{2} d\left\langle\mathbf{m}, x_{0}^{\prime}\right\rangle=\varepsilon_{i}$ for every $i \in \mathbb{N}$.

Proof. Let us prove first that (1) implies (2). Consider the family of functions $\phi: B_{S, \Delta} \rightarrow \mathbb{R}$ given by

$$
\phi\left(x^{\prime}\right)=\sum_{k=1}^{n} \gamma_{k}\left(\varepsilon_{i_{k}}+\varepsilon_{j_{k}}\right)-\sum_{k=1}^{n} \gamma_{k}\left\langle\int \varphi_{i_{k}, j_{k}, \theta_{k}} d \mathbf{m}, x^{\prime}\right\rangle,
$$

where $\gamma_{1}, \ldots, \gamma_{n}$ is a family of non negative real numbers such that $\sum_{k} \gamma_{k}=$ 1. Each such a function is convex, weak* continuous and the set of all these functions is concave. Moreover, since the functions $T_{\phi}: B_{S, \Delta} \rightarrow[0, \infty)$ given by $T_{\phi}\left(x^{\prime}\right)=\left\langle\int \sum_{k=1}^{n} \gamma_{k} \varphi_{i_{k}, j_{k}, \theta_{k}} d m, x^{\prime}\right\rangle=\int \sum_{k=1}^{n} \gamma_{k} \varphi_{i_{k}, j_{k}, \theta_{k}} d\left\langle m, x^{\prime}\right\rangle$ are weak* continuous and $B_{S, \Delta}$ is weak* compact, there exists an element $x_{\phi}^{\prime} \in B_{S, \Delta}$ such that $\left\|\left\langle\left(\gamma_{k}\right),\left(\varphi_{i_{k}, j_{k}, \theta_{k}}\right)\right\rangle\right\|_{B_{S, \Delta}}=\sup _{x^{\prime} \in B_{S, \Delta}} T_{\phi}\left(x^{\prime}\right)=T_{\phi}\left(x_{\phi}^{\prime}\right)$ and so, by the inequality en (1), we have that $\phi\left(x_{\phi}^{\prime}\right) \leq 0$. Ky Fan Lemma gives an element $x_{0}^{\prime} \in B_{S, \Delta}$ such that $\phi\left(x_{0}^{\prime}\right) \leq 0$ for all $\phi$ (see [49, Lemma 6.13.]). Consequently for every $\left\{\gamma_{k}\right\}_{k=1}^{n}$ and $\left\{\varphi_{i_{k}, j_{k}, \theta_{k}}\right\}_{k=1}^{n}$ we have that

$$
\left\langle\left(\gamma_{k}\right),\left(\varepsilon_{i_{k}}+\varepsilon_{j_{k}}\right)\right\rangle \leq\left\langle\left(\gamma_{k}\right),\left(\int \varphi_{i_{k}, j_{k}, \theta_{k}} d\left\langle\mathbf{m}, x_{0}^{\prime}\right\rangle\right)\right\rangle .
$$

In particular, for each couple $i, j \in \mathbb{N}, i \neq j$, taking $\gamma_{1}=1, \varphi_{i, j, 1}$ and $\varphi_{i, j,-1}$ we obtain

$\varepsilon_{i}+\varepsilon_{j} \leq \int\left(f_{i}^{2}+f_{j}^{2}+2 \theta f_{i} f_{j}\right) d\left\langle\mathbf{m}, x_{0}^{\prime}\right\rangle \leq \varepsilon_{i}+\varepsilon_{j}+2 \theta \int f_{i} f_{j} d\left\langle\mathbf{m}, x_{0}^{\prime}\right\rangle, \quad \theta \in\{-1,1\}$. 
Therefore, $\int f_{i} f_{j} d\left\langle\mathbf{m}, x_{0}^{\prime}\right\rangle=0$ for each pair $i \neq j$. Fix now three different indexes $\{i, j, k\} \in \mathbb{N}$. We have the inequalities

$$
\varepsilon_{r}+\varepsilon_{s} \leq \int f_{r}^{2} d\left\langle\mathbf{m}, x_{0}^{\prime}\right\rangle+\int f_{s}^{2} d\left\langle\mathbf{m}, x_{0}^{\prime}\right\rangle \leq \varepsilon_{r}+\varepsilon_{s},
$$

and so the equalities

$$
\varepsilon_{r}+\varepsilon_{s}=\int f_{r}^{2} d\left\langle\mathbf{m}, x_{0}^{\prime}\right\rangle+\int f_{s}^{2} d\left\langle\mathbf{m}, x_{0}^{\prime}\right\rangle
$$

for different $r$ and $s ; r, s \in\{i, j, k\}$. This implies that $\int f_{r}^{2} d\left\langle\mathbf{m}, x_{0}^{\prime}\right\rangle=\varepsilon_{r}$ for each $r \in\{i, j, k\}$ and finishes the proof.

The proof of $(2) \rightarrow(1)$ is a straightforward calculation; suppose that there is an element $x_{0}^{\prime}$ as in (2) and take non negative real numbers $\gamma_{1}, \ldots, \gamma_{n}$ such that $\sum_{k} \gamma_{k}=1$. Consider a sequence of functions $\left\{\varphi_{i_{k}, j_{k}, \theta_{k}}\right\}_{k=1}^{n}$. Then

$$
\begin{gathered}
\left\langle\left(\gamma_{k}\right),\left(\varepsilon_{i_{k}}+\varepsilon_{j_{k}}\right)\right\rangle=\sum_{k=1}^{n} \gamma_{k}\left(\varepsilon_{i_{k}}+\varepsilon_{j_{k}}\right)=\sum_{k=1}^{n} \gamma_{k}\left(\int f_{i_{k}}^{2} d\left\langle\mathbf{m}, x_{0}^{\prime}\right\rangle+\int f_{j_{k}}^{2} d\left\langle\mathbf{m}, x_{0}^{\prime}\right\rangle\right) \\
=\sum_{k=1}^{n} \gamma_{k}\left(\int f_{i_{k}}^{2} d\left\langle\mathbf{m}, x_{0}^{\prime}\right\rangle+\int f_{j_{k}}^{2} d\left\langle\mathbf{m}, x_{0}^{\prime}\right\rangle+2 \theta_{k} \int f_{i_{k}} f_{j_{k}} d\left\langle\mathbf{m}, x_{0}^{\prime}\right\rangle\right) \\
\left.\leq \sup _{x^{\prime} \in B_{S, \Delta}} \mid\left\langle\sum_{k=1}^{n} \gamma_{k} \int\left(f_{i_{k}}^{2}+f_{j_{k}}^{2}+2 \theta_{k} f_{i_{k}} f_{j_{k}}\right) d \mathbf{m}, x^{\prime}\right\rangle\right) \mid=\left\|\left\langle\left(\gamma_{k}\right)_{k},\left(\varphi_{i_{k}, j_{k}, \theta_{k}}\right)_{k}\right\rangle\right\|_{B_{S, \Delta}} .
\end{gathered}
$$

Remark 2.2.4. For particular cases, the condition given in part (1) of Theorem 2.2.3 can be written in a simpler way. Consider a positive vector measure $\nu: \Sigma \rightarrow \ell^{1}$ and take the sequence $\Delta$ given by $\left\{\left\|\int f_{i}^{2} d \nu\right\|\right\}_{i=1}^{\infty}$, i.e. $\varepsilon_{i}=\left\|\int f_{i}^{2} d \nu\right\|$ for all $i$. The positivity of $\nu$ and the 1-concavity of $\ell^{1}$ implies that the condition (1) in Theorem 2.2.3 is equivalent to the inequality

$$
\left\|\int f_{i}^{2} d \nu\right\|_{\ell^{1}}+\left\|\int f_{j}^{2} d \nu\right\|_{\ell^{1}} \leq\left\|\int\left(f_{i}+\theta f_{j}\right)^{2} d \nu\right\|_{\ell^{1}}
$$

for all $i, j \in \mathbb{N}, i \neq j$, and $\theta \in\{-1,1\}$.

In Examples 2.2.2, the element of the dual space that defines the measure was explicitly computed. However, sometimes this is not possible and then the characterization theorem given above becomes useful. This is the situation that is shown in the following example. 
Example 2.2.5. (1) Let $(\Omega, \Sigma, \mu)$ be a probability space. Consider a relatively weakly compact sequence $\left\{g_{k}\right\}_{k} \subset L^{1}(\mu)$ where each $g_{k}$ is positive with norm one. Let us define the vector measure $\nu: \Sigma \rightarrow \ell^{\infty}$ given by the expression $\nu(A):=\left\{\int_{A} g_{k} d \mu\right\}_{k=1}^{\infty}$. It is well defined, and since $\left\{g_{k}\right\}_{k}$ is uniformly integrable, it is countably additive. Recall that $L^{2}(\nu) \subset L^{1}(\nu)$ and note that if $f \in L^{1}(\nu)$ then $\int f d \nu=\left\{\int f g_{k} d \mu\right\}_{k} \in \ell^{\infty}$. Take a sequence $S:=\left\{f_{i}\right\}_{i} \in L^{2}(\nu)$ satisfying the following properties: $\left\|\int f_{i}^{2} d \nu\right\| \leq 1$ for all $i$ and for every finite subset $I_{0} \subseteq \mathbb{N}$ there is an index $n \in \mathbb{N}$ such that $\left\{f_{i}: i \in I_{0}\right\}$ is orthonormal in $L^{1}\left(g_{n} d \mu\right)$. Then the set $B_{S, \Delta}$ is just $B_{\left(\ell^{\infty}\right)^{\prime}} \cap\left(\left(\ell^{\infty}\right)^{\prime}\right)_{\nu}^{+}$. Let us show that this is enough to prove that condition (1) in Theorem 2.2.3 is satisfied.

Take the sequence $\Delta:=\left(\varepsilon_{i}\right)_{i}$, where $\varepsilon_{i}=1$ for every $i$. Then the set $B_{S, \Delta}$ is just $B_{\left(\ell^{\infty}\right)^{\prime}}$. For every $i, j \in \mathbb{N}, i \neq j$ and $\theta \in\{-1,1\}$, recall that

$$
\varphi_{i, j, \theta}:=\left(f_{i}+\theta f_{j}\right)^{2} .
$$

So we have to prove the inequality

$$
2 \leq\left\|\left\langle\left(\gamma_{k}\right),\left(\varphi_{i_{k}, j_{k}, \theta_{k}}\right)\right\rangle\right\|_{B_{S, \Delta}}
$$

for $\gamma_{k}>0$ such that $\sum_{k=1}^{m} \gamma_{k}=1$ and $\left\{f_{i_{k}}, f_{j_{k}} \in S: i_{k}, j_{k} \in I_{0}\right\}$ for a finite set $I_{0}$. But this is a direct consequence of the requirements of $\left\{f_{i}\right\}_{i}$ and the definition of the $\ell^{\infty}$ norm; we find an index $n$ such that

$$
\begin{gathered}
2=\sum_{k=1}^{n} \gamma_{k} \int\left(f_{i_{k}}^{2}+f_{i_{j}}^{2}\right) g_{n} d \mu=\sum_{k=1}^{n} \gamma_{k} \int\left(f_{i_{k}}+\theta_{k} f_{i_{j}}\right)^{2} g_{n} d \mu \\
=\left\langle\int \sum_{k=1}^{n} \gamma_{k} \varphi_{i_{k}, j_{k}, \theta_{k}} d \nu, e_{n}\right\rangle=\int \sum_{k=1}^{n} \gamma_{k} \varphi_{i_{k}, j_{k}, \theta_{k}} d\left\langle\nu, e_{n}\right\rangle \\
\leq\left\|\sum_{k=1}^{n} \gamma_{k} \varphi_{i_{k}, j_{k}, \theta_{k}}\right\|_{B_{S, \Delta}} .
\end{gathered}
$$

Therefore, by Theorem 2.2.3 there is an element $x^{\prime} \in B_{\left(\ell^{\infty}\right)^{\prime}} \cap\left(\left(\ell^{\infty}\right)^{\prime}\right)_{\nu}^{+}$ such that $S$ is orthonormal when considered as a sequence in $L^{2}\left(\left|\left\langle\nu, x^{\prime}\right\rangle\right|\right)$. Notice also that, although the element $x^{\prime}$ do not belong in general to $\ell^{1}$ and cannot be identified with a sequence, the measure $\left\langle\nu, x^{\prime}\right\rangle$ is absolutely continuous with respect to $\mu$, so there is an integrable function such that $\left\langle\nu, x^{\prime}\right\rangle(A)=\int_{A} h d \mu$ for every $A \in \Sigma$. 
(2) An example of the situation above is given by the following elements. Take the Lebesgue space $([0,1], \Sigma, d x)$ and the functions $g_{n}(x):=2 \sin ^{2}\left(2^{n-1} \pi x\right)$, $n \in \mathbb{N}$. Consider the Rademacher functions

$$
f_{i}(x):=\operatorname{sgn}\left(\sin \left(2^{i} \pi x\right)\right) \quad i \in \mathbb{N} .
$$

A direct computation shows that $\int f_{i}^{2} g_{n} d x=1$ for all $i, n \in \mathbb{N}$, and that for all $i, j \leq n, \int f_{i} f_{j} g_{n} d x=0$ if $i \neq j$. Consequently, the inequality in Theorem 2.2.3 is satisfied, and there is a measure $\left\langle\nu, x^{\prime}\right\rangle$ such that $\left\{f_{i}\right\}_{i}$ is a weak $\mathbf{m}$-orthonormal sequence in $L^{2}(\mathbf{m})$.

\section{$2.3 \mathrm{~m}$-Orthogonal sequences}

Let $(\Omega, \Sigma)$ be a measurable space and $X$ a Banach space. Given a vector measure $\mathbf{m}: \Sigma \rightarrow X$, consider a sequence of (non zero) real functions $\left\{f_{n}\right\}_{n}$ that are square $\mathbf{m}$-integrable. We say that it is orthogonal with respect to $\mathbf{m}$ if for every pair $j, k \in \mathbb{N}, \int f_{j} f_{k} d \mathbf{m}=0$ if $j \neq k$. Roughly speaking, it is defined by imposing simultaneously orthogonality with respect to all the elements of the family of scalar measures defined by the vector measure. This notion generalizes the usual orthogonality given by the integral with respect to a scalar measure, and provides a natural setting for studying the properties of functions that are orthogonal with respect to a family of measures. The analysis of this kind of sequences has a long mathematical history, for instance regarding orthogonal polynomials. At the end of the 19th century some relevant cases of families of polynomials that are orthogonal with respect to a large set of scalar measures -indeterminate measures - were known. The first example of such an indeterminate measure was presented by Stieltjes in 1894 (see [62]). He showed that

$$
\int_{0}^{\infty} x^{n-\log x} \sin [2 \pi \log (x)] d x=0 \quad \text { for each } \quad n=0,1,2 \ldots
$$

which implies that all the densities on the half-line

$$
d_{\lambda}(x)=\frac{(1+\lambda \sin [2 \pi \log (x)])}{x^{\log x}}, \quad \lambda \in[-1,1]
$$

have the same moments. The polynomials that are orthogonal with respect to this class of measures are a special case of the Stieltjes-Wigert polynomials. 
The study of this kind of measures was the starting point of a mathematical theory that was firstly developed by Riesz and Nevalinna and is still now a fruitful research area (see for instance $\$ 2.7$ in [63], and [8, 47, 52]). Using the family $\left(d_{\lambda}\right)_{\lambda \in[-1,1]}$ of densities, a vector measure can be defined in an easy way (see Example 2.3.2). In general this construction can be done for abstract sets of measures - for instance, parametric models in statistics-, and then to find sequences of functions that are orthogonal for all the elements of a family of measures is equivalent to the problem of finding sequences that are orthogonal with respect to a vector measure. From the point of view of the vector measure theory, orthogonality with respect to a vector measure has been studied in a series of papers in the last 10 years (see $[34,35,36,59]$ ). We define now formally the notion of $\mathbf{m}$-orthogonality with respect to a vector measure.

Definition 2.3.1. Let $(\Omega, \Sigma)$ be a measurable space and $X$ a Banach space. Given a vector measure $\mathbf{m}: \Sigma \rightarrow X$, consider a sequence of real functions $\left\{f_{i}\right\}_{i=1}^{\infty}$ that are square $\mathbf{m}$-integrable. We say that $\left\{f_{i}\right\}_{i=1}^{\infty}$ is $\mathbf{m}$-orthogonal if

$$
\int f_{i}^{2} d \mathbf{m} \neq 0, \quad \text { for all } \quad i \in \mathbb{N}, \text { and } \int f_{i} f_{j} d \mathbf{m}=0, \quad i \neq j \quad i, j \in \mathbb{N} .
$$

Furthermore, we say that $\left\{f_{n}\right\}_{n}$ is a $\mathbf{m}$-orthonormal sequence in $L^{2}(\mathbf{m})$ if for all $n \in \mathbb{N}$

$$
\left\|\int f_{n}^{2} d \mathbf{m}\right\|_{X}=1
$$

Example 2.3.2. The first example of an indeterminate measure was presented by Stieltjes in 1894 (see [62]). For each $n \in \mathbb{N}$, consider the integrals:

$$
\int_{0}^{\infty} x^{n} e^{-\ln ^{2}(x)}[1+\lambda \sin (2 \pi \ln (x))] d x=\sqrt{\pi} e^{(n+1)^{2} / 4} .
$$

If we take $|\lambda|<1$ then $\mu_{\lambda}(x)=e^{-\ln ^{2}(x)}[1+\lambda \sin (2 \pi \ln (x))]>0$ is a positive function for all $x \in\left[0, \infty\left[\right.\right.$, thus $F_{\lambda}(y)=\int_{0}^{y} e^{-\ln ^{2}(x)} x^{n}[1+\lambda \sin (2 \pi \ln (x))] d x$ is a family of non decreasing distributions with support into $[0, \infty[$ which have the same moments $S_{n}=\sqrt{\pi} e^{(n+1)^{2} / 4}$. We consider the $(n+1) \times(n+1)$ Hankel matrix:

$$
\Delta_{n}=\left[\begin{array}{cccc}
S_{0} & S_{1} & \ldots & S_{n} \\
S_{1} & \ddots & & \vdots \\
\vdots & & & \\
S_{n} & \ldots & & S_{2 n}
\end{array}\right]
$$


The sequence $\left\{S_{n}\right\}_{n=0}^{\infty}$ is defined positive if $\operatorname{det}\left(\Delta_{n}\right)>0$ for all $n \in \mathbb{N}$. We define the linear operator $\mathfrak{L}: \mathbf{P}[x] \rightarrow \mathbb{R}$ such that $\mathfrak{L}\left(Q_{n}(x)\right)=\sum_{k=0}^{\infty} a_{k} S_{k}$ where $Q_{n}(x)=\sum_{k=0}^{n} a_{k} x^{k}$.

Remark 2.3.3. If the sequence $\left\{S_{n}\right\}_{n=0}^{\infty}$ is defined positive then the polynomial

$$
P_{n}(x)=\left|\begin{array}{cccc}
S_{0} & S_{1} & \ldots & S_{n} \\
S_{1} & \ddots & & \vdots \\
\vdots & & & \\
S_{n-1} & \ldots & & S_{2 n-1} \\
1 & x & \ldots & x^{n}
\end{array}\right|
$$

satisfies that

$$
\mathfrak{L}\left(x^{k} P_{n}(x)\right)=\left\{\begin{array}{ccc}
0 & \text { if } & k<n \\
\operatorname{det}\left(\Delta_{n}\right) & \text { if } & k=n
\end{array}\right.
$$

It is immediate to prove that the sequence $\left\{S_{n}=\sqrt{\pi} e^{(n+1)^{2} / 4}\right\}_{n=0}^{\infty}$ is defined positive and:

$$
\int_{0}^{\infty} x^{k} P_{n}(x) e^{-\ln ^{2}(x)}[1+\lambda \sin (2 \pi \ln (x))] d x=\left\{\begin{array}{cll}
0 & \text { if } & k<n \\
\operatorname{det}\left(\Delta_{n}\right) & \text { if } & k=n
\end{array}\right.
$$

for every $\lambda \in \mathbb{R},|\lambda|<1$. The remark 2.3 .3 provides a procedure for building a $\mathbf{m}$-orthogonal sequence for a suitable vector measure. For instance, consider the following polynomials

$$
\begin{aligned}
p_{0}(x) & =S_{0}=2.27588, \\
p_{1}(x) & =-4.81803+2.27588 x, \\
p_{2}(x) & =183.457-139.22 x+15.059 x^{2}, \\
p_{4}(x) & =-655344 .+611203 . x-106211 . x^{2}+3438.93 x^{3}, \\
\ldots &
\end{aligned}
$$

Now we take $\Omega=(0, \infty)$ and $\Sigma$ the $\sigma$-algebra of the Lebesgue subsets of $\Omega$. We can define $\nu: \Omega \rightarrow c_{0}$ by

$$
\nu(A)=\left\{\int_{A} \frac{e^{-\ln ^{2}(x)}}{m}\left[1+\frac{1}{m+1} \sin (2 \pi \ln (x))\right] d x\right\}_{m=1}^{\infty},
$$

where $d x$ is the Lebesgue measure and $A \in \Sigma$. Using elementary integral calculus, it is easy to prove that for every $A \in \Sigma$,

$$
\lim _{m \rightarrow \infty} \int_{A} \frac{e^{-\ln ^{2}(x)}}{m}\left[1+\frac{1}{m+1} \sin (2 \pi \ln (x))\right] d x=0 .
$$


This shows that $\nu$ is well defined and so countably additive. Moreover, it is also clear that the functions $p_{l}(x) \in L^{2}(\mathbf{m})$ and they satisfy that for all $j<l$,

$$
\int_{0}^{\infty} p_{j}(x) p_{l}(x) d \nu=B_{l} \delta_{j l}
$$

where $B_{l}$ is a non null constant for all $l \in \mathbb{N}$.

Example 2.3.4. Let us provide another example of $\nu$-orthogonal sequence with respect to a vector measure $\nu$. Consider the family $\left\{P_{n, k}\right\}_{n, k}^{\infty}$ of Stieltjes-Wigert polynomials. For every $n \in \mathbb{N}$ and $k \in \mathbb{N}$,

$$
P_{n, k}(x)=\frac{(-1)^{n} q(k)^{n / 2+1 / 4}}{\sqrt{\prod_{j=1}^{k}\left(1-q(k)^{j}\right)}} \sum_{i=0}^{n}\left[\left(\begin{array}{c}
n \\
i
\end{array}\right)\right]_{q} q(k)^{\left(i^{2}\right)}(-\sqrt{q(k)} x)^{i}
$$

where $q(k)=\exp \left(-\left(2 k^{2}\right)^{-1}\right), k$ is a positive integer and we call

$$
\left[\left(\begin{array}{c}
n \\
i
\end{array}\right)\right]_{q}=\frac{\left(1-q(k)^{n}\right)\left(1-q(k)^{n-1}\right) \cdots\left(1-q(k)^{n-i+1}\right)}{(1-q(k))\left(1-q(k)^{2}\right) \cdots\left(1-q(k)^{i}\right)},
$$

as a q-binomial coefficient also called a Gaussian coefficient or Gaussian polynomial. Let us also consider the family of normalized weights $\left\{w_{k}\right\}_{k=1}^{\infty}=$ $\left\{\frac{1}{\alpha(k) \sqrt{\pi}} k x^{-k^{2} \log x}\right\}_{k=1}^{\infty}$ in $[0, \infty)$, where $\alpha(k)=e^{\frac{1}{4 k^{2}}}$. It is well-known that the family of polynomials above is orthogonal in the following sense: for a fixed $k \in \mathbb{N}$, the sequence $\left\{P_{n, k}\right\}_{n=1}^{\infty}$ is orthogonal with respect to the weight $w_{k}$, i.e.

$$
\int_{0}^{\infty} P_{n, k}(x) P_{m, k}(x) w_{k}(x) d \mu(x)=0, \quad n \neq m,
$$

see $[63,2.7]$. Consider the Lebesgue measure space $([0, \infty), \mathcal{B}, \mu)$ and take the set $\Omega_{0}=\bigcup_{k=1}^{\infty}([0, \infty) \times\{k\})$. Let us define the $\sigma$-algebra $\Sigma_{0}$ given by elements of the form $A=\bigcup_{k=1}^{\infty}\left(A_{k} \times\{k\}\right) \subseteq \Omega_{0}$, where $A_{k}$ is a Lebesgue measurable subset of $[0, \infty)$ for every $k$. Let us define the vector measure $\nu: \Sigma_{0} \rightarrow c_{0}$ as

$$
\nu(A):=\sum_{k=1}^{\infty}\left(\frac{1}{k} \int_{A_{k}} w_{k}(x) d \mu(x)\right) e_{k},
$$

where $A$ is an element of $\Sigma_{0}$ as above.

Suppose now that the polynomials $P_{n, k}$ are normalized in the Hilbert space $L^{2}\left([0, \infty), w_{k} d \mu\right)$ given by the weighted measure $w_{k}(x) d \mu(x)$. Define the functions $Q_{n, k}: \Omega_{0} \rightarrow[0, \infty)$ by

$$
Q_{n, k}\left(\left(x_{j}, j\right)\right):=k^{1 / 2} P_{n, k}\left(x_{j}\right) \delta_{k, j},
$$


for $\left(x_{j}, j\right) \in \Omega_{0}$. A careful writing of the integrals with respect to $\nu$ of the products of such functions shows that

$$
\int_{\Omega_{0}} Q_{n, k} Q_{m, s} d \nu=0
$$

whenever $n \neq m$ or $k \neq s$. To see this, just take into account that if $k \neq s$, then

$$
\begin{gathered}
\int_{\Omega_{0}} Q_{n, k} Q_{m, s} d \nu=\left(\sum_{j \neq s, k}\left(\frac{s^{1 / 2} k^{1 / 2}}{j} \int_{0}^{\infty} 0 \cdot 0 w_{j}(x) d \mu(x)\right) e_{j}\right) \\
+\left(\frac{s^{1 / 2} k^{1 / 2}}{k} \int_{0}^{\infty} P_{n, k}(x) \cdot 0 w_{k}(x) d \mu(x)\right) e_{k} \\
+\left(\frac{s^{1 / 2} k^{1 / 2}}{s} \int_{0}^{\infty} 0 \cdot P_{m, s}(x) w_{s}(x) d \mu(x)\right) e_{s}=0
\end{gathered}
$$

and for $k=s$ and $n \neq m$,

$$
\begin{gathered}
\int_{\Omega_{0}} Q_{n, k} Q_{m, k} d \nu=\left(\sum_{j \neq k}\left(\frac{k}{j} \int_{0}^{\infty} 0 \cdot 0 w_{j}(x) d \mu(x)\right) e_{j}\right) \\
+\left(\int_{0}^{\infty} P_{n, k}(x) \cdot P_{m, k}(x) w_{k}(x) d \mu(x)\right) e_{k}=0 .
\end{gathered}
$$

Therefore, every subsequence of $\left\{Q_{n, k}\right\}_{n, k=1}^{\infty}$ defines a $\nu$-orthonormal sequence.

Now we are going to provide some results on the existence of $\mathbf{m}$-orthonormal sequences in $L^{2}(\mathbf{m})$. It is easy to prove the existence of $\mathbf{m}$-orthonormal sequences of functions in any (non trivial) space of square integrable functions with respect to a vector measure.

Lemma 2.3.5. Suppose that there is a sequence $\left\{A_{n}\right\}_{n=1}^{\infty}$ into $\Sigma$ of disjoint non $\|\mathbf{m}\|-$ null sets. Then there is a $\mathbf{m}$-orthonormal sequence in $L^{2}(\mathbf{m})$.

Proof. The characteristic functions $\chi_{A_{n}} \in L^{2}(\mathbf{m})$, for every $n \in \mathbb{N}$. Moreover, since $\|\mathbf{m}\|(A) \neq 0$, there is a subset $B_{n} \subset A$ such that $\left\|\mathbf{m}\left(B_{n}\right)\right\|_{X}>0$. Let us define $f_{n}=\frac{\chi_{B_{n}}}{\left\|\mathbf{m}\left(B_{n}\right)\right\|^{1 / 2}}, \quad n \in \mathbb{N}$. Then

$$
\int f_{n}^{2} d \mathbf{m}=\int \frac{\chi_{B_{n}}^{2}}{\left\|\mathbf{m}\left(B_{n}\right)\right\|} d \mathbf{m}=\frac{1}{\left\|\mathbf{m}\left(B_{n}\right)\right\|} \int \chi_{B_{n}} d \mathbf{m}=\frac{\mathbf{m}\left(B_{n}\right)}{\left\|\mathbf{m}\left(B_{n}\right)\right\|} \neq 0 .
$$

On the other hand, if $n \neq k$ for $n, k \in \mathbb{N}$, it is clear that $\int f_{n} f_{k} d \mathbf{m}=0$, since $B_{n} \cap B_{k}=\emptyset$, and the result is obtained. 
It is clear that an $\mathbf{m}$-orthonormal sequence also is also orthogonal for each associated $\mathbf{m}$-positive measure $\left\langle\mathbf{m}, x^{\prime}\right\rangle$. The notion of $\mathbf{m}$-orthonormal sequence is the natural generalization of the concept of orthonormal sequence in a Hilbert space $L^{2}(\mu)$ and has been studied in [48, 59]. As the reader will see, the results on almost everywhere convergence that will be shown in next chapter prove the ones that hold for the case of series in Hilbert spaces. However, the m-orthonormality requirement for a sequence of functions in the non scalar case introduces a strong restriction, in particular regarding completeness of the orthogonal sequence. An orthonormal set $\Psi$ is said to be complete if there exists no other orthonormal set containing $\Psi$, that is, $\Psi$ must be a maximal orthonormal set. It is easy to prove that an orthonormal set $\Psi$ is complete if and only if for any $f$ such that $f$ is orthogonal to $\Psi, f$ must be zero. Let us show this fact with an easy construction. Suppose that $\left(\Omega_{0}, \Sigma_{0}, \mu_{0}\right)$ is a probability measure space, and consider a complete orthonormal sequence $\left\{g_{i}\right\}_{i=1}^{\infty}$ in $L^{2}\left(\mu_{0}\right)$ such that $g_{1}=$ $\chi_{\Omega_{0}}$. Suppose that $\left\{g_{i}\right\}_{i=1}^{\infty}$ is also $\mathbf{m}_{0}$-orthonormal sequence for a countably additive measure $\mathbf{m}_{0}: \Sigma_{0} \rightarrow X$ that is absolutely continuous with respect to $\mu_{0}$. Then every measure $\left(\mathbf{m}_{0}\right)_{x^{\prime}}, x^{\prime} \in X^{\prime}$, is $\mu_{0}$-continuous, and there is a function $h_{x^{\prime}} \in L^{1}\left(\mu_{0}\right)$ such that $d\left(\mathbf{m}_{0}\right)_{x^{\prime}}=h_{x^{\prime}} d \mu_{0}$. For every $k \geq 2$,

$$
0=\left\langle\int g_{1} g_{k} d \mathbf{m}_{0}, x^{\prime}\right\rangle=\int g_{k} h_{x^{\prime}} d \mu_{0} .
$$

If moreover $h_{x^{\prime}} \in L^{2}\left(\mu_{0}\right)$, since the sequence $\left\{g_{i}\right\}_{i=1}^{\infty}$ is complete, the equalities above imply $h_{x^{\prime}}=r\left(x^{\prime}\right) \chi_{\Omega_{0}}$ for a real number $r\left(x^{\prime}\right)$. Therefore, if we assume that for every $x^{\prime} \in X^{\prime}$ the corresponding Radon-Nikodým derivative belongs to $L^{2}\left(\mu_{0}\right)$, we obtain that

$$
\left(\mathbf{m}_{0}\right)_{x^{\prime}}(A)=r\left(x^{\prime}\right) \mu_{0}(A), \quad A \in \Sigma_{0}, \quad x^{\prime} \in X^{\prime} .
$$

This relation establishes a strong restriction on $\mathbf{m}_{0}$. For instance, suppose that $X$ is a Banach space with an unconditional basis $\left\{e_{i}\right\}_{i=1}^{\infty}$. Then $\mathbf{m}_{0}(A)=$ $\sum_{i=1}^{\infty}\left\langle\mathbf{m}_{0}(A), e_{i}^{\prime}\right\rangle e_{i}$, where $\left\{e_{i}^{\prime}\right\}_{i=1}^{\infty}$ are the corresponding biorthogonal functionals and $A \in \Sigma_{0}$. In this case the relation above implies that

$$
\mathbf{m}_{0}(A)=\left(\sum_{i=1}^{\infty} r\left(e_{i}^{\prime}\right) e_{i}\right) \mu_{0}(A)
$$

for every $A \in \Sigma_{0}$, i.e. $\mathbf{m}_{0}$ can be in fact considered as a scalar positive measure.

Remark 2.3.6. The above argument shows that in general we cannot expect completeness for $\mathbf{m}$-orthonormal sequence of functions, although under certain 
(strong) assumptions it is possible to obtain $\mathbf{m}$-orthonormal basis for $L^{2}(\mathbf{m})$ (see [48]). Thus, although the results that we present in what follows can be used to obtain information about standard orthonormal sequences $\left\{f_{i}\right\}_{i=1}^{\infty}$ in Hilbert spaces $L^{2}(\mu)$, the procedure of splitting the scalar measure $\mu$ into a vector measure $\mathbf{m}$ preserving orthonormality is essentially limited by a certain non completeness assumption for $\left\{f_{i}\right\}_{i=1}^{\infty}$.

The next result is a direct consequence of the Kadec and Pelczynski process for obtaining disjoint sequences -see Chapter 1.3 for notation-. It provides a method for, given a convenient sequence $\left\{f_{n}\right\}_{n}$, finding a disjoint (and then $\mathbf{m}$-orthogonal) subsequence of $\left\{f_{n}\right\}_{n}$.

Corollary 2.3.7. Let $H$ be Hilbert space which is also a Banach lattice and $\mathbf{m}: \Sigma \rightarrow H$ a positive vector measure such that $\|\mathbf{m}\|(\Omega)=1$. Take a Rybakov control measure $\mu=\left|x_{0}^{\prime} \mathbf{m}\right|$ for $\mathbf{m}$ with $\left\|x_{0}^{\prime}\right\|=1$. If $\left\{f_{n}\right\}_{n} \subset L^{2}(\mathbf{m})$ is such that $\left\|f_{n}\right\|_{L^{2}(m)}=1$ for all $n$ and $\left\{f_{n}\right\}_{n} \nsubseteq M_{L^{2}(\mathbf{m})}(\varepsilon)$ for all $\varepsilon>0$ then there exists a subsequence $\left\{h_{k}\right\}_{k=1}^{\infty} \subset L^{2}(\mathbf{m})$ such that $\left|h_{k}\right| \leq\left|f_{n_{k}}\right|$ for all $k$ and $\left\{h_{k}\right\}_{k=1}^{\infty}$ is a $\mathbf{m}$-orthogonal sequence in $L^{2}(\mathbf{m})$. Moreover, $\left\{f_{n_{k}}\right\}_{k=1}^{\infty}$ and $\left\{h_{k}\right\}_{k=1}^{\infty}$ are equivalent unconditional basic sequences that satisfies $\lim _{k \rightarrow \infty}\left\|f_{n_{k}}-h_{k}\right\|_{L^{2}(\mathbf{m})}=$ 0 .

The proof is a direct application of Theorem 1.3.3. Note that $\lim _{k \rightarrow \infty} \| f_{n_{k}}-$ $h_{k} \|_{L^{2}(\mathbf{m})}=0$ and $\left\|f_{n_{k}}\right\|_{L^{2}(\mathbf{m})}=1$ for all $k$ implies that $\int h_{k}^{2} d \mathbf{m} \neq 0$ for large enough $k$.

\subsection{Strongly $\mathrm{m}$-orthogonality}

In this section we present the last notion of orthogonality, that is the most restrictive. In what follows we will establish the conditions that are required in order to construct a strongly $\mathbf{m}$-orthonormal system.

Definition 2.4.1. Let us consider a separable Hilbert space $H$ with an orthonormal sequence $\left\{e_{i}\right\}_{i=1}^{\infty}$ and a countably additive vector measure $\mathbf{m}: \Sigma \rightarrow$ $H$. We say that $\left\{f_{i}\right\}_{i=1}^{\infty} \subset L^{2}(\mathbf{m})$ is a strongly $\mathbf{m}$-orthogonal sequence if $\int f_{i} f_{j} d \mathbf{m}=\delta_{i j} e_{i} k_{i}, k_{i}>0$. If $k_{i}=1$ for every $i$, we say that it is a strongly m-orthonormal sequence. 
It is clear that if $\left\{f_{n}\right\}_{n}$ is a strongly $\mathbf{m}$-orthogonal sequence then $\left\{f_{n}\right\}_{n}$ is a $\mathbf{m}$-orthogonal sequence and therefore, in the case when $H$ is also a Banach lattice and $\mathbf{m}$ is positive, $\left\{f_{n}\right\}_{n}$ is weak $\mathbf{m}$-orthogonal. The converse is not true.

Example 2.4.2. Let $r_{k}(x)=\operatorname{sgn}\left[\sin \left(2^{k-1} x\right)\right]$ be the Rademacher function of period $2 \pi$, defined at the interval $E_{k}=[2(k-1) \pi, 2 k \pi], k \in \mathbb{N}$. We define the following countably additive vector measure,

$$
\mathbf{m}(A):=\sum_{k=1}^{\infty} \frac{(-1)}{2^{k}}\left(\int_{A \cap E_{k}} r_{k} d \mu\right) e_{k} \in \ell^{2} .
$$

Note that if $f \in L^{1}(\mathbf{m})$ then $\int f d \mathbf{m}=\left(-\frac{1}{2^{k}} \int_{E_{k}} f r_{k} d \mu\right)_{k} \in \ell^{2}$. We consider the next sequence of functions

$$
\begin{aligned}
f_{1}(x)= & \sin x \cdot \chi_{[\pi, 2 \pi]} \\
f_{2}(x)= & \sin 2 x \cdot\left(\chi_{[0,2 \pi]}+\chi_{\left[\frac{7}{2} \pi, 4 \pi\right]}\right) \\
f_{3}(x)= & \sin 4 x \cdot\left(\chi_{[0,4 \pi]}+\chi_{\left[\frac{23}{4} \pi, 6 \pi\right]}\right) \\
& \cdots \\
f_{k}(x)= & \sin 2^{k-1} x \cdot\left(\chi_{[0,2(k-1) \pi]}+\chi_{\left[\left(2 k-\frac{1}{2^{k-1}}\right) \pi, 2 k \pi\right]}\right), \quad k \geq 2 .
\end{aligned}
$$

These family of functions of $L^{2}(\mathbf{m})$ can be used to define a strongly $\mathbf{m}$-orthogonal

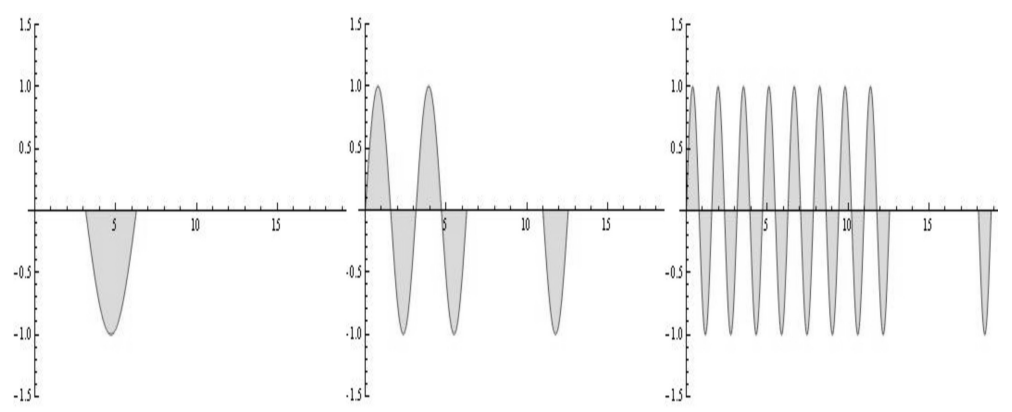

Figure 2.1: Functions $f_{1}(x), f_{2}(x)$ and $f_{3}(x)$.

sequence (see figure 2.1), since

$$
\begin{aligned}
\left\langle\int f_{n}^{2} d \mathbf{m}, e_{n}\right\rangle & =-\frac{1}{2^{n}} \int_{E_{n}} f_{n}^{2} r_{n} d \mu=\frac{\pi}{2^{2 n}} \\
\left\langle\int f_{n}^{2} d \mathbf{m}, e_{k}\right\rangle & =-\frac{1}{2^{k}} \int_{E_{k}} f_{n}^{2} r_{k} d \mu=0, \quad \forall k \neq n \\
\left\langle\int f_{n} f_{m} d \mathbf{m}, e_{k}\right\rangle & =-\frac{1}{2^{k}} \int_{E_{k}} f_{n} f_{m} r_{k} d \mu=0, \quad \text { for } n \neq m \text { and } \forall k
\end{aligned}
$$


If we define

$$
F_{n}(x)=\frac{2^{n}}{\sqrt{\pi}} f_{n}(x)
$$

we obtain

$$
\int F_{i}^{2} d \mathbf{m}=e_{i}, \quad \forall i \in \mathbb{N} \quad \text { and } \quad \int F_{i} F_{j} d \mathbf{m}=0, \quad \forall i, j \in \mathbb{N}, \quad i \neq j .
$$

Now we adapt a well know result of Bessaga and Pelczynski (see for instance [24], Ch.V) to the context of sequences of functions on spaces of integrable functions with respect to a vector measure in the following sense. We want to identify when a sequence $\left\{\int f_{n}^{2} d \mathbf{m}\right\}_{n=1}^{\infty} \subset H$ is a basic sequence of $H$. This will be the main tool for obtaining what we have denoted strongly $\mathbf{m}$-orthogonal sequences. The first requirement in Corollary 2.4.3 is obviously satisfied in the case of $\mathbf{m}$-orthonormal sequences. The second condition constitutes the key of the problem.

Corollary 2.4.3. Let $\mathbf{m}: \Sigma \longrightarrow H$ be a vector measure, and consider the basis $\left\{e_{n}\right\}_{n=1}^{\infty}$ of $H$. Let $\left\{f_{n}\right\}_{n=1}^{\infty}$ be a sequence in $L^{2}(\mathbf{m})$. If the sequence $\left\{\int f_{n}^{2} d \mathbf{m}\right\}_{n=1}^{\infty}$ satisfies

(1) $\inf _{n}\left\|\int f_{n}^{2} d \mathbf{m}\right\|_{H}=\varepsilon>0$, and

(2) $\lim _{n}\left\langle e_{k}, \int f_{n}^{2} d \mathbf{m}\right\rangle=0, \quad k \in \mathbb{N}$,

then $\left\{\int f_{n}^{2} d \mathbf{m}\right\}_{n=1}^{\infty}$ has a subsequence which is a basic sequence, moreover it is equivalent to a block basic sequence of $\left\{e_{n}\right\}_{n=1}^{\infty}$.

The proof of this corollary is an immediate consequence of Theorem 3 [7]. Let us highlight with an example the geometrical meaning of this condition by showing a particular sequence of functions that satisfies Remark 1.2.5. This is in a sense the canonical situation, involving disjointness.

Example 2.4.4. Let $(\Omega, \Sigma, \mu)$ be a probability measure space. Let us consider the following vector measure $\mathbf{m}: \Sigma \rightarrow \ell^{2}$,

$$
\mathbf{m}(A):=\sum_{i=1}^{\infty} \mu\left(A \cap A_{i}\right) e_{i} \in \ell^{2}, \quad A \in \Sigma,
$$

where $\left\{A_{i}\right\}_{i=1}^{\infty}$ is a disjoint measurable partition of $\Omega$, with $\mu\left(A_{i}\right) \neq 0$ for all $i \in$ $\mathbb{N}$. Notice that $\int f^{2} d \mathbf{m}=\sum_{i=1}^{\infty}\left(\int_{A_{i}} f^{2} d \mu\right) e_{i} \in \ell^{2}$ for all $f \in L^{2}(\mathbf{m})$. Consider 
the sequence of functions $\left\{f_{n}\right\}_{n=1}^{\infty}$ in $L^{2}(\mathbf{m})$, defined by $f_{n}:=\chi_{A_{n}} /\left(\mu\left(A_{n}\right)\right)^{1 / 2}$. For every $k \in \mathbb{N}$, the following holds:

$$
\begin{aligned}
\lim _{n}\left\langle e_{k}, \int f_{n}^{2} d \mathbf{m}\right\rangle= & \lim _{n}\left\langle e_{k}, \sum_{i=1}^{\infty}\left(\int_{A_{i}} f_{n}^{2} d \mu\right) e_{i}\right\rangle=\lim _{n} \int_{A_{k}} f_{n}^{2} d \mu=0 . \\
& \left\|\int f_{n}^{2} d m\right\|_{\ell^{2}}=1 \text { for all } n .
\end{aligned}
$$

Therefore, condition (2) of Corollary 2.4.3 is fulfilled in this example: the role of disjointness is clear. The geometrical meaning of this condition by showing a particular sequence of functions that satisfies Remark 1.2.5. This is in a sense the canonical situation, involving disjointness.

For the case of vector measures $\mathbf{m}$ having a compact integration operator, we provide an easy argument showing that there are no strong $\mathbf{m}$-orthogonal sequences in $L^{2}(\mathbf{m})$ (see Proposition 3.48 [49]). In particular, this shows that the existence of such sequences is a pure vector measure phenomenon. In fact, this property is closely related to the existence of strongly $\mathbf{m}$-orthonormal sequences. Compactness of the integration map is nowadays well characterized. It is a very strong property in the sense that it implies that the space $L^{1}(\mathbf{m})$ is isomorphic to an $L^{1}$ space of an scalar measure. The result can be found in Proposition 3.48. [49] (see also the references there in). The following necessary condition for the compactness of the integration operator shows this.

Lemma 2.4.5. Let $\mathbf{m}: \Sigma \longrightarrow H$ be an countably additive vector measure. If there exists a strongly $\mathbf{m}$-orthonormal sequence in $L^{2}(\mathbf{m})$, then the integration operator $I_{\mathbf{m}}: L^{1}(\mathbf{m}) \longrightarrow H$ is not compact.

Proof. Let us consider an orthonormal sequence $\left\{e_{i}\right\}_{i}$ in $H$ and let $\left\{f_{i}\right\}_{i}$ be a strongly $\mathbf{m}$-orthogonal sequence in $L^{2}(\mathbf{m})$. Then $\int f_{i} f_{j} d \mathbf{m}=\delta_{i j} e_{i}$, an thus

$$
\int f_{i}^{2} d \mathbf{m}=e_{i}=I_{\mathbf{m}}\left(f_{i}^{2}\right) \text {. }
$$

Therefore $\left\{f_{i}^{2}\right\}_{i=1}^{\infty} \subset B_{L^{1}(\mathbf{m})}$ and so the sequence

$$
\left\{I_{\mathbf{m}}\left(f_{i}^{2}\right)\right\}_{i=1}^{\infty} \subset I_{\mathbf{m}}\left(B_{L^{1}(\mathbf{m})}\right) \subset \overline{I_{\mathbf{m}}\left(B_{L^{1}(\mathbf{m})}\right)}
$$

does not admit any convergent sub-sequence, from what follows that $\overline{I_{\mathbf{m}}\left(B_{L^{1}(\mathbf{m})}\right)}$ is not compact and so, $I_{\mathbf{m}}\left(B_{L^{1}(\mathbf{m})}\right)$ is not relatively compact, what allows us to conclude that $I_{\mathbf{m}}$ is not compact. 
Therefore, if $I_{\mathbf{m}}: L^{1}(\mathbf{m}) \longrightarrow H$ is compact then no strongly $\mathbf{m}$-orthonormal sequence exists in $L^{2}(\mathbf{m})$.

The following theorem gives a necessary condition -and, in a sense, also a sufficient condition- for the existence of strongly orthonormal sequences in a space of functions $L^{2}(\mathbf{m})$ starting from a given $\mathbf{m}$-orthonormal sequence. In general, it is not possible to find a subsequence satisfying this condition. However, as the reader will see, it is possible to find a vector measure $\mathbf{m}^{*}$ satisfying that $L^{2}(\mathbf{m})=L^{2}\left(\mathbf{m}^{*}\right)$ and with respect to which there is a subsequence that is strongly $\mathbf{m}^{*}$-orthonormal. We need first the following lemma, which proof is elementary.

Lemma 2.4.6. Let $\mathbf{m}: \Sigma \rightarrow \ell^{2}$ be a vector measure. Let $\varphi: \ell^{2} \rightarrow H$ be an isomorphism, where $H$ is a separable Hilbert space, and consider the vector measure $\mathbf{m}^{*}=\varphi \circ \mathbf{m}$. Then the spaces $L^{2}(\mathbf{m})$ and $L^{2}\left(\mathbf{m}^{*}\right)$ are isomorphic, and for every $f \in L^{2}(\mathbf{m}), \int f^{2} d \mathbf{m}^{*}=\varphi\left(\int f^{2} d \mathbf{m}\right)$.

We need the following formal requirement for the elements of the sequence $\left\{f_{n}\right\}_{n=1}^{\infty}$ in the result below. We say that a function $f \in L^{2}(\mathbf{m})$ is normed by the integral if $\|f\|_{L^{2}(\mathbf{m})}=\left\|\int f^{2} d \mathbf{m}\right\|^{1 / 2}$. This happens for instance when the vector measure is positive - i.e. it has its range in the positive cone of a Banach lattice-, see [23] or [49, Lemma 3.13]. We impose this condition for the aim of clarity, although the result could be adapted using a convenient renorming process in order to avoid this requirement.

Theorem 2.4.7. Let us consider a vector measure $\boldsymbol{m}: \Sigma \rightarrow \ell^{2}$ and an $\boldsymbol{m}$ orthonormal sequence $\left\{f_{n}\right\}_{n=1}^{\infty}$ of functions in $L^{2}(\boldsymbol{m})$ that are normed by the integrals. Let $\left\{e_{n}\right\}_{n=1}^{\infty}$ be the canonical basis of $\ell^{2}$. If

$$
\lim _{n}\left\langle e_{k}, \int f_{n}^{2} d \boldsymbol{m}\right\rangle=0
$$

for every $k \in \mathbb{N}$, then there exists a subsequence $\left\{f_{n_{k}}\right\}_{k=1}^{\infty}$ of $\left\{f_{n}\right\}_{n=1}^{\infty}$ and a Hilbert space valued measure $\boldsymbol{m}^{*}: \Sigma \rightarrow H$ such that $\left\{f_{n_{k}}\right\}_{k=1}^{\infty}$ is a strongly $\boldsymbol{m}^{*}$-orthonormal. Moreover, $\boldsymbol{m}^{*}$ can be chosen as $\boldsymbol{m}^{*}=\phi \circ \boldsymbol{m}$ for some Banach space isomorphism $\phi$ from $\ell^{2}$ onto $H$.

Proof. Consider an $\mathbf{m}$-orthonormal sequence $\left\{f_{n}\right\}_{n=1}^{\infty}$ in $L^{2}(\mathbf{m})$ and the sequence of integrals $\left\{\int f_{n}^{2} d \mathbf{m}\right\}_{n=1}^{\infty}$. As an application of Corollary 2.4.3, we 
get a subsequence $\left\{\int f_{n_{k}}^{2} d \mathbf{m}\right\}_{k=1}^{\infty}$ that is equivalent to a block basic sequence $\left\{e_{n_{k}}^{\prime}\right\}_{k=1}^{\infty}$ of the canonical basis of $H$. Recall that following the notation given in the Preliminaries $\alpha_{i, j}$ are the constants that appear in the definition of the block basic sequence. Associated to the block basic sequence obtained there is an isomorphism $\varphi$

$$
A:=\overline{\operatorname{span}\left(e_{n_{k}}^{\prime}\right)^{H}} \stackrel{\varphi}{\longrightarrow} B:=\overline{\operatorname{span}\left(\int f_{n_{k}}^{2} d \mathbf{m}\right)} \ell^{\ell^{2}}
$$

such that $\varphi\left(e_{n_{k}}^{\prime}\right):=\int f_{n_{k}}^{2} d \mathbf{m}, k \in \mathbb{N}$.

We can suppose without loss of generality that the elements of the sequence $\left\{e_{n_{k}}^{\prime}\right\}_{k=1}^{\infty}$ have norm one. To see this, it is enough to consider the following inequalities. First note that there are positive constants $k$ and $K$ such that for every $n \in \mathbb{N}, k=k\left\|\int f_{n_{k}}^{2} d \mathbf{m}\right\| \leq\left\|e_{n_{k}}^{\prime}\right\| \leq K\left\|\int f_{n_{k}}^{2} d \mathbf{m}\right\|=K$ as a consequence of the existence of the isomorphism $\varphi$. Let $\left\{\lambda_{i}\right\}_{i=1}^{\infty}$ be a sequence of real numbers. Then

$$
\left\|\sum_{i=1}^{\infty} \lambda_{i} \frac{e_{i}^{\prime}}{\left\|e_{i}^{\prime}\right\|}\right\|_{2}^{2}=\sum_{i=1}^{\infty} \frac{\left|\lambda_{i}\right|^{2}\left(\sum_{j=p_{i}}^{q_{i}}\left|\alpha_{i, j}\right|^{2}\right)}{\left\|e_{i}^{\prime}\right\|^{2}}=\sum_{i=1}^{\infty} \sum_{j=p_{i}}^{q_{i}} \frac{\left|\lambda_{i}\right|^{2}\left|\alpha_{i, j}\right|^{2}}{\left\|e_{i}^{\prime}\right\|^{2}} .
$$

The existence of an upper and a lower bound for the real numbers $\left\|e_{i}^{\prime}\right\|$ given above provides the equivalence between this quantity and

$$
\left\|\sum_{i=1}^{\infty} \lambda_{i} e_{i}^{\prime}\right\|_{2}^{2}
$$

for every sequence of real numbers $\left\{\lambda_{i}\right\}_{i=1}^{\infty}$.

Since each closed subspace of a Hilbert space is complemented, there is a subspace $B^{c}$ such that

$$
\ell^{2}=B \oplus_{2} B^{c}
$$

isometrically, where this direct sum space is considered as a Hilbert space (with the adequate Hilbert space norm). We write $P_{B}$ and $P_{B^{c}}$ for the corresponding projections. Let us consider the linear map $\phi:=\varphi^{-1} \oplus I d$, where $I d: B^{c} \rightarrow B^{c}$ is the identity map.

$$
B \oplus_{2} B^{c} \stackrel{\phi}{\longrightarrow} A \oplus_{2} B^{c} .
$$

Note that $H:=A \oplus_{2} B^{c}$ is a Hilbert space when the scalar product

$$
\left\langle x+y, x^{\prime}+y^{\prime}\right\rangle_{H}=\left\langle x, x^{\prime}\right\rangle_{H}+\left\langle y, y^{\prime}\right\rangle_{H}, \quad x+y, x^{\prime}+y^{\prime} \in A \oplus_{2} B^{c}
$$


is considered. Obviously, $\phi$ is an isomorphism. Let us consider also the vector measure $\mathbf{m}^{*}:=\phi \circ \mathbf{m}$,

$$
\Sigma \stackrel{m}{\longrightarrow} \ell^{2} \stackrel{\phi}{\longrightarrow} A \oplus_{2} B^{c} .
$$

By Lemma 2.4.6, $L^{2}(\mathbf{m})=L^{2}(\phi \circ \mathbf{m})=L^{2}\left(\mathbf{m}^{*}\right)$. Let us show that $\left\{f_{n_{k}}\right\}_{k=1}^{\infty}$ is a strongly $\mathbf{m}^{*}$-orthonormal sequence. We consider the orthonormal sequence $\left\{\left(e_{n_{k}}^{\prime}, 0\right)\right\}_{k=1}^{\infty}$ in $H$. The first condition in the definition of strongly orthonormal sequence is fulfilled, since

$$
\begin{gathered}
\int f_{n_{k}}^{2} d \mathbf{m}^{*}=\int f_{n_{k}}^{2} d(\phi \circ \mathbf{m})=\phi\left(P_{B}\left(\int f_{n_{k}}^{2} d \mathbf{m}\right), P_{B^{c}}\left(\int f_{n_{k}}^{2} d \mathbf{m}\right)\right) \\
=\left(\varphi^{-1}\left(\int f_{n_{k}}^{2} d \mathbf{m}\right), 0\right)=\left(e_{n_{k}}^{\prime}, 0\right)
\end{gathered}
$$

for every $k \in \mathbb{N}$. The second one is given by the following calculations. For $k \neq l$,

$$
\left\|\int f_{n_{k}} f_{n_{l}} d \mathbf{m}^{*}\right\|=\left\|\int f_{n_{k}} f_{n_{l}} d(\phi \circ \mathbf{m})\right\|=\left\|\phi\left(\int f_{n_{k}} f_{n_{l}} d \mathbf{m}\right)\right\|=\|\phi(0)\|=0,
$$

since $\phi$ is continuous and $\left\{f_{n_{k}}\right\}_{k=1}^{\infty}$ is a $\mathbf{m}$-orthonormal sequence. Thus

$$
\int f_{n_{k}} f_{n_{l}} d \mathbf{m}^{*}=0
$$

This proof the theorem.

Corollary 2.4.8. Let $\mathbf{m}: \sum \longrightarrow \Upsilon(\Upsilon$ a separable Hilbert space) and let $\left\{f_{n}\right\}_{n=1}^{\infty}$ be an $\mathbf{m}$-orthonormal sequence in $L^{2}(\mathbf{m})$, such that $\left\{\int_{\Omega} f_{n}^{2} d \mathbf{m}\right\}_{n=1}^{\infty}$ is weakly null in $H$. Then there is a subsequence $\left\{f_{n_{k}}\right\}_{k=1}^{\infty}$ and a complete orthonormal basis $\left\{n_{j}\right\}_{j=1}^{\infty}$ for $\Upsilon$, such that $\left\{f_{n(k)}\right\}_{k=1}^{\infty}$ is strongly $\mathbf{m}$-orthonormal with the basis $\left\{n_{j}\right\}_{j=1}^{\infty}$.

Remark 2.4.9. In a certain sense, the converse of the theorem above also holds. Take as the vector measure $\mathbf{m}^{*}$ the measure $\mathbf{m}$ itself with values in $\ell^{2}$ and consider the canonical basis $\left\{e_{n}\right\}_{n=1}^{\infty}$. Clearly, every strongly $\mathbf{m}$-orthonormal sequence is $\mathbf{m}$-orthonormal and satisfies the condition $\lim _{n}\left\langle e_{k}, \int f_{n}^{2} d \mathbf{m}^{*}\right\rangle=0$, since

$$
\left\langle e_{k}, \int f_{n}^{2} d \mathbf{m}^{*}\right\rangle=\left\langle e_{k}, e_{n}\right\rangle=0, \quad k \neq n .
$$




\section{Chapter 3}

\section{The Menchoff-Rademacher Theorem for $L^{2}(\mathbf{m})$}

One of the key issues that arose in the late XIX, it is when the development of a Fourier series of a continuous function converges pointwise to the function. The first result that answered partially this question was given by Dirichlet showing that if a function has bounded variation then its Fourier series converges pointwise everywhere to the local average of the function. If we change the summation method we can obtain the convergence almost everywhere. Later Fejer showed that if we consider the sums of Cesàro sums instead of the ordinary sums then the Fourier series of a continuous function converges pointwise everywhere to the function. Luzin conjectured that the Fourier series of a Lebesgue-measurable function of $L^{2}$ converges almost everywhere. Kolmogorov showed an example of a function in $L^{1}$ whose Fourier series diverges almost everywhere. Finally Carleson proved the conjecture of Luzin in 1966. In this section we analyze almost everywhere convergence of weak m-orthogonal sequences, and we obtain a version of a Mechoff-Rademacher Theorem.

Let $(\Omega, \Sigma, \mu)$ be a finite measure space, and consider an orthonormal sequence $\left\{f_{i}\right\}_{i}$ of real functions in $L^{2}(\mu)$ and a sequence of real numbers $\left\{a_{i}\right\}_{i}$. The Menchoff-Rademacher Theorem (see $[45,46,51])$ is the main result concerning 
$\mu$-almost everywhere convergence of the norm convergent series $\sum_{i=1}^{\infty} a_{i} f_{i}$, and establishes that it converges $\mu$-a.e. if $\sum_{i=1}^{\infty}\left|a_{i}\right|^{2} \log ^{2}(i+1)<\infty$. Although for particular (even complete) orthonormal sequences this result can be improved (for instance, it is enough that $\sum_{i=1}^{\infty}\left|a_{i}\right|^{2}<\infty$ for the Haar and the trigonometric sequences, see 1.6.1 in [1] and [11]), it is optimal if we consider any orthonormal sequence.

In this chapter we prove a Menchoff-Rademacher type theorem on almost everywhere convergence of series in $L^{2}(\mathbf{m})$ and we analyze some particular cases regarding $c_{0}$-sums of Lebesgue spaces. Although several properties and applications of orthogonal series with respect to a vector measure are known $([35,36,48])$, the question of the almost everywhere convergence of series defined by such functions has not been studied yet. Following this research and as an application of the criterion for weak orthogonality, the results that we present provide also reasonable answers to the problems concerning almost everywhere convergence of (strongly orthogonal) series that appear in [48, 59]. From the methodological point of view, we follow the technique that is used in [64] to study the almost everywhere convergence of series. In Section III.H of this book it is shown that there exists a deep link between the evaluation of 2 -summing norms for a special class of operators between sequence spaces and the problems concerning almost everywhere convergence of series; the origin of this idea can be already found in [6, Section 4] (see also [50]). Recently, related techniques have been used in $[14,20,21]$, for instance for proving generalizations of the Menchoff-Rademacher Theorem for vector valued Banach function spaces $([21])$. We explain the required version of this argument for our work in the proof of Theorem 3.2.2 and we establish the requirements for obtaining the almost everywhere convergence of series defined by weak orthogonal sequences. Finally, we provide a technique to construct non trivial examples of weak m-orthogonal sequences $\left\{f_{i}\right\}_{i=0}^{\infty}$ in particular Banach lattices such that the requirement $\sum_{i=1}^{\infty} a_{i}^{2}<\infty$ on the sequence of scalar coefficients implies the a.e. convergence of the series $\sum_{i=1}^{\infty} a_{i} f_{i}$. 


\subsection{About almost everywhere convergence of se- quences in $L^{2}(\mathbf{m})$}

Let $(\Omega, \Sigma)$ be a measureble space, $X$ a Banach lattice and $\mathbf{m}: \Sigma \rightarrow X$ a vector measure. Let us establish first some general results on $\mathbf{m}$-a.e. convergence of sequences in $L^{2}(\mathbf{m})$. Throughout this section $\left\{f_{n}\right\}_{n}$ will be a sequence of measurable functions of integrable square with respect to a vector measure $\mathbf{m}$.

Definition 3.1.1. Given a function $f \in L^{2}(\mathbf{m})$, we define the set

$$
E_{n}(\alpha)=\left\{\omega \in \Omega:\left|f_{n}(\omega)-f(\omega)\right| \geq \alpha\right\} .
$$

The sequence $\left\{f_{n}\right\}_{n=1}^{\infty}$ is said to convergence in vector measure $\mathbf{m}$ to $f$ if

$$
\lim _{n \rightarrow \infty}\|\mathbf{m}\|\left(E_{n}(\alpha)\right)=0
$$

for each $\alpha$. Since $\mathbf{m}$ is a positive vector measure, the condition (3.1.2) is equivalent to the following expression

$$
\lim _{n \rightarrow \infty}\left\|\mathbf{m}\left(E_{n}(\alpha)\right)\right\|_{X}=0 .
$$

The following two results can be found in Section $7[5]$ for scalar measures.

Lemma 3.1.2. Let $\left\{f_{n}\right\}_{n=1}^{\infty}$ be a sequence of measurable real-valued functions which converge to a measurable function $f$ in $L^{2}(\mathbf{m})$, then $\left\{f_{n}\right\}_{n=1}^{\infty}$ converges to $f$ in vector measure $\mathbf{m}$.

Proof. Since the inequalities

$\left\|f_{n}-f\right\|_{L^{2}(\mathbf{m})} \geq\left\|\left(f_{n}-f\right) \chi_{E_{n}(\alpha)}\right\|_{L^{2}(\mathbf{m})} \geq\left\|\alpha \chi_{E_{n}(\alpha)}\right\|_{L^{2}(\mathbf{m})}=|\alpha|\|\mathbf{m}\|\left(E_{n}(\alpha)\right)^{1 / 2}$.

hold for any constant $K$ and it is clear that if $f_{n} \rightarrow f$ in $L^{2}(\mathbf{m})$ then the last term converges to 0 .

The above result can be obtained immediately if we apply the following implications. Let $\mu$ be a Rybakov measure. Then

- $f_{n} \rightarrow f$ in vector measure $\mathbf{m}$ if and only if $f_{n} \rightarrow f$ in $\mu$ measure.

- If $f_{n} \rightarrow f$ in $L^{2}(\mathbf{m})$ then $f_{n} \rightarrow f$ in $L^{2}(\mu)$. 
Definition 3.1.3. The sequence $\left\{f_{n}\right\}_{n=1}^{\infty}$ of measurable real-valued functions converges $\mathbf{m}$-almost everywhere to a measurable function $f$ if there exists a set $M$ in $\Omega$ with $\|\mathbf{m}\|(M)=0$, such that for every $\varepsilon>0$ and $\omega \in \Omega-M$ there exists a natural number $N(\varepsilon, \omega)$ such that if $n \geq N(\varepsilon, \omega)$ then $\left|f_{n}(\omega)-f(\omega)\right|<\varepsilon$.

Proposition 3.1.4. Let $\left\{f_{n}\right\}_{n=1}^{\infty}$ be a sequence of real-valued measurable functions such that converges to a measurable function $f$ in the norm of $L^{2}(\mathbf{m})$. Then there is a subsequence which converges $\mathbf{m}$-almost everywhere to $f$.

Proof. By Lemma 3.1.6 we have that if $\left\{f_{n}\right\}_{n=1}^{\infty}$ converge to a measurable function $f$ in the norm of $L^{2}(\mathbf{m})$ then $\left\{f_{n}\right\}_{n=1}^{\infty}$ converges to $f$ in vector measure m. Now we proof that there exists a subsequence that converges $\mathbf{m}$-almost everywhere to $f$. Select a subsequence $\left\{f_{n_{k}}\right\}$ of $\left\{f_{n}\right\}$ such that the set

$$
E_{k}=\left\{\omega \in \Omega:\left|f_{n_{k}}(\omega)-f(\omega)\right| \geq 2^{-k}\right\}
$$

satisfies $\|\mathbf{m}\|\left(E_{k}\right)<2^{-k}$. Let $F_{k}=\bigcup_{j=k}^{\infty} E_{j}$ so that $F_{k} \in \Sigma$ then

$$
\|\mathbf{m}\|\left(F_{k}\right)=\|\mathbf{m}\|\left(\bigcup_{j=k}^{\infty} E_{j}\right) \leq \sum_{j=k}^{\infty}\|\mathbf{m}\|\left(E_{j}\right) \leq \sum_{j=k}^{\infty} 2^{-j}=2^{-(k-1)} .
$$

If $i \geq k$ and $\omega$ is not into $F_{k}$ then $\left|f_{n_{i}}(\omega)-f(\omega)\right| \leq 2^{-k}$. Let $F=\bigcap_{k=1}^{\infty} F_{k}$. Then $\|\mathbf{m}\|(F)=0$. From the argument just given it follows that $\left\{f_{n_{k}}\right\}$ converges $\mathbf{m}$-almost everywhere to the measurable real-valued function $f$.

In what follows we analyze some properties of the integrals in the subspaces generated by $\mathbf{m}-$ orthogonal sequences.

Lemma 3.1.5. Let $\left\{f_{i}\right\}_{i=1}^{\infty}$ be an $\mathbf{m}$-orthonormal sequence. For all $I_{0} \subset \mathbb{N}$ and $\left(\lambda_{i}\right)_{i \in I_{0}} \subset \mathbb{R}$ such that $g=\sum_{i \in I_{0}} \lambda_{i} f_{i}$ converges in $L^{2}(\mathbf{m})$, then $\int g f_{j} d \mathbf{m}=0$ if $j \in \mathbb{N} \backslash I_{0}$

Proposition 3.1.6. Let $Y(\nu)$ be a Banach function space, $\mathbf{m}: \Sigma \rightarrow Y(\nu)$ a positive vector measure and $\left\{f_{i}\right\}_{i=1}^{\infty}$ a $\mathbf{m}$-orthogonal sequence and let $f_{0}$ be a measurable function such that $f_{0} \in L^{2}(\mathbf{m})$. Then if $\left\|f_{0}-\sum_{i=1}^{n} \lambda_{i} f_{i}\right\|_{L^{2}(\mathbf{m})}$ converges to 0 we have:

(1) $\int\left(\sum_{i=1}^{n} \lambda_{i}^{2} f_{i}^{2}\right) d \mathbf{m}$ is an increasing positive sequence that converges to $\int f_{0}^{2} d \mathbf{m}$ in norm and so $\nu$-almost everywhere. 
(2) $\int\left(\sum_{i=1}^{n} \lambda_{i} f_{i}-f_{0}\right)^{2} d \mathbf{m}$ is a decreasing positive sequence that converges 0 in norm and so $\nu$-almost everywhere.

(3) $\int \sum_{i=1}^{n} \lambda_{i} f_{i} d \mathbf{m}$ converges to $\int f_{0} d \mathbf{m} \nu$-almost everywhere.

Proof. We suppose that $\left\|f_{0}-\sum_{i=1}^{n} \lambda_{i} f_{i}\right\|_{L^{2}(\mathbf{m})}$ converges to 0 . Then

$$
\begin{gathered}
\int\left|\sum_{i=1}^{n} \lambda_{i} f_{i}-f_{0}\right|^{2} d \mathbf{m}=\int\left(\sum_{i=1}^{n} \lambda_{i} f_{i}-f_{0}\right)^{2} d \mathbf{m} \\
=\sum_{i=1}^{n} \int \lambda_{i}^{2} f_{i}^{2} d \mathbf{m}+\int f_{0}^{2} d \mathbf{m}-2 \sum_{i=1}^{n} \lambda_{i} \int f_{i} f_{0} d \mathbf{m} \\
=\sum_{i=1}^{n} \int \lambda_{i}^{2} f_{i}^{2} d \mathbf{m}+\int f_{0}^{2} d \mathbf{m} \\
-2 \sum_{i=1}^{n} \lambda_{i} \int f_{i}\left(\sum_{k=1}^{n} \lambda_{k} f_{k}\right) d \mathbf{m}-2 \sum_{i=1}^{n} \lambda_{i} \int f_{i}\left(\sum_{k=n+1}^{\infty} \lambda_{k} f_{k}\right) d \mathbf{m} \\
=\sum_{i=1}^{n} \int \lambda_{i}^{2} f_{i}^{2} d \mathbf{m}+\int f_{0}^{2} d \mathbf{m}-2 \sum_{i=1}^{n} \int \lambda_{i}^{2} f_{i}^{2} d \mathbf{m} \\
=\int f_{0}^{2} d \mathbf{m}-\sum_{i=1}^{n} \lambda_{i}^{2} \int f_{i}^{2} d \mathbf{m} .
\end{gathered}
$$

Hence, we have that $\left\{\sum_{i=1}^{n} \lambda_{i}^{2} \int f_{i}^{2} d \mathbf{m}\right\}_{n}$ is an increasing sequence that converges to $\int f_{0}^{2} d \mathbf{m}$ in norm and so $\nu$-almost everywhere, and also we have that (2) holds. In order to prove (3), recall that if $T: X(\nu) \rightarrow Y(\nu)$ is a positive operator between Banach function spaces, we always have that if $0 \leq f, g \in X(\mu)$, $f \cdot g \in X(\mu)$ then $T(f \cdot g) \leq T\left(f^{2}\right)^{1 / 2} \cdot T\left(g^{2}\right)^{1 / 2}$. Since the integration operator with respect to $\mathbf{m}$ is positive, just consider the (pointwise) inequalities

$$
\begin{gathered}
\left(\int\left(f_{0}-\sum_{i=1}^{n} \lambda_{i} f_{i}\right)^{2} d \mathbf{m}\right)^{\frac{1}{2}}(\mathbf{m}(\Omega))^{\frac{1}{2}} \geq\left|\int\left(f_{0}-\sum_{i=1}^{n} \lambda_{i} f_{i}\right) d \mathbf{m}\right| \\
=\left|\int f_{0} d \mathbf{m}-\int\left(\sum_{i=1}^{n} \lambda_{i} f_{i}\right) d \mathbf{m}\right|
\end{gathered}
$$

so the sequence $\left\{\int \sum_{i=1}^{n} \lambda_{i} f_{i} d \mathbf{m}\right\}_{n}$ converges $\nu$-almost everywhere to $\int f_{0} d \mathbf{m}$.

Remark 3.1.7. Let $Y(\nu)$ be a Banach function space, $\mathbf{m}: \Sigma \rightarrow Y(\nu)$ a vector measure and and $\left\{f_{i}\right\}_{i=1}^{\infty}$ a $\mathbf{m}$-orthogonal sequence and let $f_{0}$ be a measurable function such that $f_{0} \in L^{2}(\mathbf{m})$. If we consider an $\mathbf{m}$-orthogonal subsequence $\left\{f_{i_{k}}\right\}_{k}$ of $\left\{f_{i}\right\}_{i}$ such that $\left\|f_{0}-\sum_{i=1}^{n} \lambda_{i} f_{i_{k}}\right\|_{L^{2}(\mathbf{m})}$ converges to 0 then the Proposition 3.1.6 holds. 


\subsection{The Menchoff-Rademacher Theorem for weak m-orthogonal sequences}

In this section we study the almost everywhere convergence of functional series defined by (real valued) functions that are weak $\mathbf{m}$-orthogonal for a vector measure $\mathbf{m}$. The vector measure is supposed to be positive through all the chapter. We develop a technique for generalizing the arguments that prove the Menchoff-Rademacher Theorem to our setting (see [64, III.H.22] for the scalar measure case). It provides the adequate elements for proving more specialized versions of this theorem depending on the properties of the space where the vector measure is defined. Let $\mathbf{m}: \Sigma \rightarrow X$ be a positive vector measure over $(\Omega, \Sigma)$. Our aim is to obtain conditions on a weak $\mathbf{m}$-orthogonal sequence $\left\{f_{i}\right\}_{i}$ and a sequence of real numbers $\left\{a_{i}\right\}_{i}$ to assure $\left\langle\mathbf{m}, x^{\prime}\right\rangle$-almost everywhere convergence of the series $\sum_{i=1}^{\infty} a_{i} f_{i}$ for a certain $x^{\prime} \in\left(X^{\prime}\right)_{\mathbf{m}}^{+}$.

Let $x^{\prime} \in\left(X^{\prime}\right)_{\mathbf{m}}^{+}$. Let $\left\{f_{i}\right\}_{i}$ be a sequence that is orthogonal with respect to $\left\langle\mathbf{m}, x^{\prime}\right\rangle$. Consider Banach sequence space $L$ over the standard measure space on $\mathbb{N}$ with canonical (normalized) basis $\left\{e_{i}\right\}_{i}$. If $s$ is a natural number, we write $\Phi_{s}$ for the function $\Phi_{s}: \Omega \rightarrow L$ given by the formula

$$
\Phi_{s}(\omega):=\sum_{i=1}^{s} f_{i}(\omega) e_{i}, \quad \omega \in \Omega .
$$

Note that this function belongs to the Bochner space $L^{2}\left(\left\langle\mathbf{m}, x^{\prime}\right\rangle, L\right)$ for every $x^{\prime} \in\left(X^{\prime}\right)_{\mathbf{m}}^{+}$, since $f_{i} \in L^{2}(\mathbf{m})$ for every $i \in \mathbb{N}$ can be considered as a (class of) function(s) of $L^{2}\left(\left\langle\mathbf{m}, x^{\prime}\right\rangle\right)$.

Definition 3.2.1. Consider a Banach sequence space $L$ and a sequence of real numbers $a:=\left\{a_{i}\right\}_{i}$. We denote by $\sigma_{a, L}$ the operator $\sigma_{a, L}: L \rightarrow \ell^{\infty}$ given by

$$
\sigma_{a, L}\left(\left(\lambda_{i}\right)_{i}\right):=\left\{\left(\sum_{i=1}^{n} a_{i} \lambda_{i}\right)\right\}_{n=1}^{\infty}, \quad\left\{\lambda_{i}\right\}_{i} \in L,
$$

if it is well defined and continuous. We also write $\sigma_{a, L}^{N}$ for the operator $\sigma_{a, L}^{N}$ : $L \rightarrow \ell^{\infty}$ defined as $\sigma_{a(N), L}$, where $a(N)_{i}=a_{i}$ for every $i \geq N$, and 0 otherwise.

Theorem 3.2.2. Let $x^{\prime} \in B_{X^{\prime}} \cap\left(X^{\prime}\right)_{\mathbf{m}}^{+}$. Consider a sequence of real numbers $a=\left\{a_{i}\right\}_{i=1}^{\infty}$ and a sequence $\left\{f_{i}\right\}_{i=1}^{\infty} \in L^{2}(\mathbf{m})$ that is orthogonal with respect to 
3.2 The Menchoff-Rademacher Theorem for weak $\mathbf{m}$-orthogonal sequences

$\left\langle\mathbf{m}, x^{\prime}\right\rangle$. Let $L$ be a 2 -concave sequence space and let $M$ be a sequence space such that $\left(L^{\prime}\right)_{[2]}=M^{\prime}$. Suppose that

(1) there is a constant $K$ such that $\left\|\left\{\left\langle\int f_{i}^{2} d \mathbf{m}, x^{\prime}\right\rangle\right\}_{i=1}^{s}\right\|_{M}<K$ for every $s \in \mathbb{N}$ and

(2) the operators $\sigma_{a, L}^{N}: L \rightarrow \ell^{\infty}$ are 2 -summing and $\lim _{N \rightarrow \infty} \pi_{2}\left(\sigma_{a, L}^{N}\right)=0$.

Then the series $\sum_{i=1}^{\infty} a_{i} f_{i}$ converges $\left\langle\mathbf{m}, x^{\prime}\right\rangle$-a.e.

Proof. First we prove the following claim: Let $Y$ be a Banach space and let $T: L \rightarrow Y$ be a 2 -summing operator, then for every natural number $s$,

$$
\left\|T \Phi_{s}\right\|_{L^{2}\left(\left\langle\mathbf{m}, x^{\prime}\right\rangle, Y\right)} \leq \pi_{2}(T)\left\|\left\{\left\langle\int f_{i}^{2} d \mathbf{m}, x^{\prime}\right\rangle\right\}_{i=1}^{s}\right\|_{M}^{\frac{1}{2}} .
$$

To prove this, first note that the elements of the space $\left(L^{\prime}\right)_{[2]}$ are sequences $\tau=\left\{\tau_{i}\right\}_{i}$ that satisfy that there is a sequence $z^{\prime}=\left\{z_{i}^{\prime}\right\}_{i} \in L^{\prime}$ such that for every $i \in \mathbb{N},\left|z_{i}^{\prime}\right|^{2}=\left|\tau_{i}\right|$. Since $L^{\prime}$ is 2-convex (see [42, Proposition 1.d.4(iii)]), $\left(L^{\prime}\right)_{[2]}$ is a Banach space with norm $\|\tau\|_{\left(L^{\prime}\right)_{[2]}}:=\left\|\left\{\tau_{i}\right\}_{i}\right\|_{\left(L^{\prime}\right)_{[2]}}=\left\|\left(\left|\tau_{i}\right|^{1 / 2}\right)_{i}\right\|_{L^{\prime}}^{2}$ (recall that we assume for simplicity that the 2-concavity constant of $L$ is 1 and then the 2-convexity constant of $L^{\prime}$ also equals 1 ; see [18] and [42, Proposition 1.d.4(iii)]). Since $T$ is 2 -summing, a direct calculation (see [64, Proposition III.F.33,b)]) gives

$$
\left\|T \Phi_{s}\right\|_{L^{2}\left(\left\langle\mathbf{m}, x^{\prime}\right\rangle, Y\right)}^{2} \leq \pi_{2}^{2}(T) \sup _{z^{\prime} \in B_{L^{\prime}}} \int\left|\left\langle\Phi_{s}(\omega), z^{\prime}\right\rangle\right|^{2} d\left\langle\mathbf{m}, x^{\prime}\right\rangle .
$$

Since $\left\{f_{i}\right\}_{i}$ is orthogonal with respect to $\left\langle\mathbf{m}, x^{\prime}\right\rangle$, the inequality above can be written as

$$
\left\|T \Phi_{s}\right\|_{L^{2}\left(\left\langle\mathbf{m}, x^{\prime}\right\rangle, Y\right)}^{2} \leq \pi_{2}^{2}(T) \sup _{z^{\prime} \in B_{L^{\prime}}} \sum_{i=1}^{s}\left|z_{i}^{\prime}\right|^{2} \int f_{i}^{2} d\left\langle\mathbf{m}, x^{\prime}\right\rangle ;
$$

all the integrals in this expression are positive, so we also obtain

$$
\begin{gathered}
\sup _{z^{\prime} \in B_{L^{\prime}}} \sum_{i=1}^{s}\left|z_{i}^{\prime}\right|^{2} \int f_{i}^{2} d\left\langle\mathbf{m}, x^{\prime}\right\rangle=\sup _{\tau \in B_{\left(L^{\prime}\right)} \sum_{[2]}} \sum_{i=1}^{s}\left|\tau_{i}\right| \int f_{i}^{2} d\left\langle\mathbf{m}, x^{\prime}\right\rangle \\
=\left\|\left(\left\langle\int f_{i}^{2} d \mathbf{m}, x^{\prime}\right\rangle\right)_{i=1}^{s}\right\|_{M}<K .
\end{gathered}
$$

This gives the desired inequality and proves the claim.

Now we just need to show that the requirements for the operators $\sigma_{a, L}^{N}$ are enough to apply an standard almost everywhere convergence criterion (see 
for instance [64, III.H.22]). A direct calculation shows that for each natural numbers $N$ and $s$, consider the function $g_{N, s}: \Omega \rightarrow[0, \infty)$ defined by

$$
g_{N, s}(\omega)=\left\|\sigma_{a, L}^{N}\left(\Phi_{s}(\omega)\right)\right\|_{\infty}=\max _{n=N, \ldots, s}\left|\sum_{i=N}^{n} a_{i} f_{i}(\omega)\right| .
$$

Applying the claim for $T=\sigma_{a, L}^{N}$ we have that

$$
\left\|g_{N, s}\right\|_{L^{2}\left(\left\langle\mathbf{m}, x^{\prime}\right\rangle\right)}=\left\|\sigma_{a, L}^{N} \Phi_{s}\right\|_{L^{2}\left(\left\langle\mathbf{m}, x^{\prime}\right\rangle, \ell^{\infty}\right)} \leq \pi_{2}\left(\sigma_{a, L}^{N}\right) K^{1 / 2} .
$$

Fixing $N$, the sequence $\left\{g_{N, s}^{2}\right\}_{s}$ increases to $h_{N}^{2}$ where

$$
h_{N}(\omega)=\sup _{n \geq N}\left|\sum_{i=N}^{n} a_{i} f_{i}(\omega)\right| .
$$

Then, the monotone convergence theorem gives

$$
\int h_{N}^{2} d\left\langle\mathbf{m}, x^{\prime}\right\rangle=\lim _{s \rightarrow \infty} \int g_{N, s}^{2} d\left\langle\mathbf{m}, x^{\prime}\right\rangle \leq \pi_{2}^{2}\left(\sigma_{a, L}^{N}\right) K
$$

and so $h_{N} \in L^{2}\left(\left\langle\mathbf{m}, x^{\prime}\right\rangle\right)$ with $\left\|h_{N}\right\|_{L^{2}\left(\left\langle\mathbf{m}, x^{\prime}\right\rangle\right)} \leq \pi_{2}\left(\sigma_{a, L}^{N}\right) K^{1 / 2}$. Thus, condition (2) in the statement of the theorem implies that the sequence $\left\{h_{N}\right\}_{N=1}^{\infty}$ converges to 0 in $L^{2}\left(\left\langle\mathbf{m}, x^{\prime}\right\rangle\right)$, and then there is a subsequence that converges $\left\langle\mathbf{m}, x^{\prime}\right\rangle$-a.e. to 0 . This clearly implies that the sequence itself converges $\left\langle\mathbf{m}, x^{\prime}\right\rangle$ a.e. to 0 since it is decreasing. Hence, for $\left\langle\mathbf{m}, x^{\prime}\right\rangle$-a.e. every $\omega \in \Omega$ and $\varepsilon>0$ there is a natural number $R$ such that for every $N \geq R$,

$$
\left|\sum_{i=1}^{N} a_{i} f_{i}(\omega)-\sum_{i=1}^{R-1} a_{i} f_{i}(\omega)\right| \leq \sup _{n \geq R}\left|\sum_{i=R}^{n} a_{i} f_{i}(\omega)\right|=h_{R}(\omega)<\varepsilon
$$

and then the series $\sum_{i=1}^{\infty} a_{i} f_{i}(\omega)$ converges $\left\langle\mathbf{m}, x^{\prime}\right\rangle$-a.e.

Note that the almost everywhere convergence with respect to a measure defined by a positive element $x^{\prime}$ do not provide $\mathbf{m}$-almost everywhere convergence, since such measures are not in general Rybakov measures. This means that such a measure can have more null sets in the $\sigma$-algebra $\Sigma$.

Remark 3.2.3. The requirements on $L$ in Theorem 3.2 .2 show that the problem of the almost everywhere convergence of weak $\mathbf{m}$-orthogonal series is closely related to the calculus of estimates of 2-summing norms for the operators $\sigma_{a, L}^{N}$ : $L \rightarrow \ell^{\infty}$ for suitable sequence spaces $L$. The canonical examples of such spaces are sequence spaces that satisfy that the inclusions $\ell^{1} \subseteq L \subseteq \ell^{2}$ are well defined 
and continuous; take $L=\ell^{p}$ for $1 \leq p \leq 2$. Then $\left(L^{\prime}\right)_{[2]}=\left(\ell^{p^{\prime}}\right)_{[2]}=\ell^{p^{\prime} / 2}$. If we consider $1 \leq q \leq \infty$ such that $1 / q=1 / p-1 / p^{\prime}$, then the space $M$ satisfying $M^{\prime}=\left(\left(\ell^{p}\right)^{\prime}\right)_{[2]}$ is $\ell^{q}\left(c_{0}\right.$ if $\left.p=2\right)$. In the following section we develop the case $p=1$, for which $\left(L^{\prime}\right)_{[2]}=\left(\ell^{\infty}\right)_{[2]}=\ell^{\infty}$, and then $M=\ell^{1}$.

Let us finish this section by giving two estimates for these norms (we give the estimates for $\sigma_{a, L}$, the ones for $\sigma_{a, L}^{N}$ are obtained with the same arguments). Note that a direct application of the following inequalities to Theorem 3.2.2 provides formulas involving the sequence " $a$ " that can be directly computed.

(1) The first one comes from an application of Grothendieck's Theorem and can be used for the case of operators $\sigma_{a, \ell^{1}}: \ell^{1} \rightarrow \ell^{\infty}$ that are still continuous when defined as $\sigma_{a, \ell^{2}}: \ell^{2} \rightarrow \ell^{\infty}$. Consider a sequence $a \in \ell^{2}$. In this case we can write a factorization of $\sigma_{a, \ell^{1}}$ as $\sigma_{a, \ell^{2}} \circ i d$, where $i d: \ell^{1} \rightarrow \ell^{2}$ is the inclusion map. This map is 1-summing (see for instance 17.14 in [19]), which implies that it is also 2-summing, and thus $\sigma_{a, \ell^{1}}$ is so. Moreover,

$$
\pi_{2}\left(\sigma_{a, \ell^{1}}\right) \leq \pi_{2}(i d)\left\|\sigma_{a, \ell^{2}}\right\|_{\ell^{1}} \leq\left(\sum_{i=1}^{\infty} a_{i}^{2}\right)^{\frac{1}{2}},
$$

(see e.g Exercise 11.5 in [19] for the estimate of $\pi_{2}(i d)$ ). Of course, the same argument can be used for general $\sigma_{a, L}: L \rightarrow \ell^{\infty}$ whenever it can be factored through $i d: \ell^{1} \rightarrow \ell^{2}$. This estimate gives the following result: If $\left\{f_{i}\right\}_{i} \subset L^{2}(\mathbf{m})$ is orthogonal with respect to $\left\langle\mathbf{m}, x^{\prime}\right\rangle$ for some $x^{\prime} \in$ $B_{X^{\prime}} \cap\left(X^{\prime}\right)_{\mathbf{m}}^{+}$and $\left(\int f_{i}^{2} d\left\langle\mathbf{m}, x^{\prime}\right\rangle\right)_{i} \in \ell^{1}$, then $\sum_{i \geq 1} a_{i} f_{i}$ converges $\left\langle\mathbf{m}, x^{\prime}\right\rangle$ a.e. for all $\left\{a_{i}\right\}_{i} \in \ell^{2}$.

(2) For the second one the argument is similar, but using the fact that the operator $\sigma_{b, \ell^{1}}$ is integral -we write $\iota(T)$ for the integral norm of an operator $T$-, where $b=\{1 / \log (i+1)\}_{i}$ (see the reference to the BennetMaurey-Nahoum Theorem in [20, Section 4], and [19] for the definition and properties of integral operators). Suppose that the sequence "a" satisfies that

$$
\left\|\left\{a_{i} \log (i+1)\right\}_{i}\right\|_{L^{\prime}}<\infty .
$$

This requirement is the natural generalization of the Menchoff-Rademacher condition for a.e. convergence. We can obtain the factorization through $\ell^{1}$ given by $\sigma_{a, L}=\sigma_{b, \ell^{1}} \circ D_{c}$, where $D_{c}$ is the diagonal operator defined 
by the sequence $c_{i}=a_{i} \log (i+1)$, since

$$
\pi_{2}\left(\sigma_{a, L}\right) \leq \iota\left(\sigma_{a, L}\right) \leq \iota\left(\sigma_{b, \ell^{1}}\right)\left\|D_{c}\right\| \leq \iota\left(\sigma_{b, \ell^{1}}\right)\left\|\left\{a_{i} \log (i+1)\right\}_{i}\right\|_{L^{\prime}} .
$$

The same factorization can be used for every sequence $b$ such that $\sigma_{b, \ell^{1}}$ is 2-summing. This estimate gives the following result: If $\left\{f_{i}\right\}_{i} \subset L^{2}(\mathbf{m})$ is orthogonal with respect to $\left\langle\mathbf{m}, x^{\prime}\right\rangle$ for some $x^{\prime} \in B_{X^{\prime}} \cap\left(X^{\prime}\right)_{\mathbf{m}}^{+}$and $\left\{\int f_{i}^{2} d\left\langle\mathbf{m}, x^{\prime}\right\rangle\right\}_{i} \in \ell^{r}$ for $1<r \leq \infty$, then $\sum_{i \geq 1} a_{i} f_{i}$ converges $\left\langle\mathbf{m}, x^{\prime}\right\rangle$-a.e. for all $\left\{a_{i}\right\}_{i} \subset \mathbb{R}$ such that $\left(a_{i} \log (i+1)\right) \in \ell^{q}$ for $q=\frac{2 r}{r-1}$.

The following results combine Theorem 2.2.3, Theorem 3.2.2 and the remark above to give several criteria for $\left\langle\mathbf{m}, x^{\prime}\right\rangle$-a.e. convergence of sequences in $L^{2}(\mathbf{m})$.

Corollary 3.2.4. Let $\left\{f_{i}\right\}_{i=1}^{\infty}$ be a sequence in $L^{2}(\mathbf{m})$. Let $L$ be a 2 -concave sequence space and let $M$ be a sequence space such that $\left(L^{\prime}\right)_{[2]}=M^{\prime}$. Let $\Delta=$ $\left\{\varepsilon_{i}\right\}_{i}$ a sequence such that $\left\{\varepsilon_{i}\right\}_{i=1}^{s} \in M$ for all $s \in \mathbb{N}$ with $\sup _{s}\left\|\left\{\varepsilon_{i}\right\}_{i=1}^{\infty}\right\|<\infty$ and let $a=\left\{a_{i}\right\}_{i}$ be a sequence of real numbers, let $B_{S, \Delta}$ be the convex weak* compact subset (see 2.2.1 for definition) and suppose that

(1) the operators $\sigma_{a, L}^{N}: L \rightarrow \ell^{\infty}$ are 2 -summing and $\lim _{N \rightarrow \infty} \pi_{2}\left(\sigma_{a, L}^{N}\right)=0$ and

(2) for every finite sequence of non negative real numbers $\left\{\gamma_{k}\right\}_{k}$ such that $\sum_{k} \gamma_{k}=1$, indexes $i_{k}, j_{k} \in \mathbb{N}, i_{k} \neq j_{k}$, and $\theta_{k} \in\{-1,1\}$,

$$
\left\langle\left(\gamma_{k}\right),\left(\varepsilon_{i_{k}}+\varepsilon_{j_{k}}\right)\right\rangle \leq\left\|\left\langle\left(\gamma_{k}\right),\left(\varphi_{i_{k}, j_{k}, \theta_{k}}\right)\right\rangle\right\|_{B_{S, \Delta}} .
$$

Then there is an element $x^{\prime} \in X^{\prime}$ such that the sequence $\left\{\frac{f_{i}}{\sqrt{\varepsilon_{i}}}\right\}_{i}$ is orthonormal in $L^{2}\left(\left\langle\mathbf{m}, x^{\prime}\right\rangle\right)$ and the series $\sum_{i=1}^{\infty} a_{i} f_{i}$ converges $\left\langle\mathbf{m}, x^{\prime}\right\rangle$-a.e.

The same result can be written in terms of the properties of the sequence of the integrals.

Corollary 3.2.5. Let $\left\{f_{i}\right\}_{i=1}^{\infty}$ be a sequence in $L^{2}(\mathbf{m})$ such that $\left\{\left\|\int f_{i}^{2} d \mathbf{m}\right\|\right\}_{i=1}^{s} \in$ $M$ for all $s \in \mathbb{N}$ with $\sup _{s}\left\|\left\{\left\|\int f_{i}^{2} d \mathbf{m}\right\|\right\}_{i=1}^{\infty}\right\|<\infty$. Let $L$ be a 2-concave sequence space and let $M$ be a sequence space such that $\left(L^{\prime}\right)_{[2]}=M^{\prime}$. Let $a=\left\{a_{i}\right\}_{i}$ be a sequence of real numbers and suppose that

(1) the operators $\sigma_{a, L}^{N}: L \rightarrow \ell^{\infty}$ are 2 -summing and $\lim _{N \rightarrow \infty} \pi_{2}\left(\sigma_{a, L}^{N}\right)=0$ and 
(2) there exists $\Delta=\left\{\varepsilon_{i}\right\}_{i}$ such for every finite sequence of non negative real numbers $\left\{\gamma_{k}\right\}_{k}$ such that $\sum_{k} \gamma_{k}=1$, indexes $i_{k}, j_{k} \in \mathbb{N}, i_{k} \neq j_{k}$, and $\theta_{k} \in\{-1,1\}$,

$$
\left\langle\left(\gamma_{k}\right),\left(\varepsilon_{i_{k}}+\varepsilon_{j_{k}}\right)\right\rangle \leq\left\|\left\langle\left(\gamma_{k}\right),\left(\varphi_{i_{k}, j_{k}, \theta_{k}}\right)\right\rangle\right\|_{B_{S, \Delta}} .
$$

Then there is an element $x^{\prime} \in X^{\prime}$ such that the sequence $\left\{\frac{f_{i}}{\sqrt{\varepsilon_{i}}}\right\}_{i}$ is orthonormal in $L^{2}\left(\left\langle\mathbf{m}, x^{\prime}\right\rangle\right)$ and the series $\sum_{i=1}^{\infty} a_{i} f_{i}$ converges $\left\langle\mathbf{m}, x^{\prime}\right\rangle$-a.e.

Example 3.2.6. (1) For the case of $\ell^{1}$-valued measures and $L=\ell^{2}$, we obtain using Remark 2.2.4 that the result is similar to the one that holds for scalar measures. Let $\mathbf{m}: \Sigma \rightarrow \ell^{1}$ be a positive vector measure and consider a sequence $\left\{f_{i}\right\}_{i} \subseteq L^{2}(\mathbf{m})$ of norm one functions. Let $a=\left\{a_{i}\right\}_{i}$ be a sequence of real numbers and suppose that

(a) the operators $\sigma_{a, \ell^{2}}^{N}: \ell^{2} \rightarrow \ell^{\infty}$ are 2 -summing and $\lim _{N \rightarrow \infty} \pi_{2}\left(\sigma_{a, \ell^{2}}^{N}\right)=$ 0 , and

(b) $\sqrt{2} \leq\left\|f_{i}+\theta f_{j}\right\|_{L^{2}(\mathbf{m})}$ for all $i, j \in \mathbb{N}, i \neq j$, and $\theta \in\{-1,1\}$.

Then Corollary 3.2.5 gives an element $x^{\prime} \in \ell^{\infty}$ such that the sequence $\left\{f_{i}\right\}_{i}$ is orthonormal in $L^{2}\left(\left\langle\mathbf{m}, x^{\prime}\right\rangle\right)$ and the series $\sum_{i=1}^{\infty} a_{i} f_{i}$ converges $\left\langle\mathbf{m}, x^{\prime}\right\rangle$ a.e. For example, a direct calculation shows that for $x^{\prime}=(1,1,1, \ldots)$, the result holds.

(2) Let us show an application regarding Example 2.2.5 also for $L=\ell^{2}$. Let $\left\{e_{i}\right\}_{i}$ be the canonical basic sequence in $\ell^{\infty}$ and take an $\ell^{\infty}$ valued (countably additive) positive vector measure $\nu$, a sequence of functions $\left\{f_{i}\right\}_{i} \in L^{2}(\mathbf{m})$ such that $\int f_{i}^{2} d\left\langle\nu, e_{j}\right\rangle=1$ for every $i, j \in \mathbb{N}$ and a sequence $a=\left\{a_{i}\right\}_{i}$ such that the operators $\sigma_{a, \ell^{2}}^{N}: \ell^{2} \rightarrow \ell^{\infty}$ are 2-summing with $\lim _{N \rightarrow \infty} \pi_{2}\left(\sigma_{a, \ell^{2}}^{N}\right)=0$. Assume also that for every finite sequence of non negative real numbers $\left\{\gamma_{k}\right\}_{k}$ such that $\sum_{k} \gamma_{k}=1$, indexes $i_{k}, j_{k} \in \mathbb{N}$, $i_{k} \neq j_{k}$, and $\theta_{k} \in\{-1,1\}$,

$$
2 \leq \sup _{i}\left|\int \sum_{k} \gamma_{k}\left(f_{i_{k}}+\theta_{k} f_{j_{k}}\right)^{2} d\left\langle\nu, e_{i}\right\rangle\right|
$$

Then by Corollary 3.2.5 there is an element $x^{\prime} \in\left(\ell^{\infty}\right)^{\prime}$ such that the sequence $\left\{f_{i}\right\}_{i}$ is orthonormal in $L^{2}\left(\left\langle\mathbf{m}, x^{\prime}\right\rangle\right)$ and the series $\sum_{i=1}^{\infty} a_{i} f_{i}$ converges $\left\langle\mathbf{m}, x^{\prime}\right\rangle$-a.e. 


\subsection{Almost everywhere convergence in $c_{0}$-sums of $L^{2}(\mu)$ spaces}

In this section we use the representation of $\ell$-sums of $L^{2}$-spaces as spaces $L^{2}(\mathbf{m})$ for a suitable $\mathbf{m}$ to apply our results. In particular, we develop the case of $c_{0^{-}}$ sums of $L^{2}$-spaces.

Let be $(\Omega, \Sigma, \mu)$ a finite measure space and consider a disjoint partition $\left\{E_{i}\right\}_{i} \subset \Sigma$ of $\Omega$. Consider the sequence space $c_{0}$. We define a countably additive vector measure $\mathbf{n}: \Sigma \rightarrow c_{0}$ by

$$
\mathbf{n}(E):=\sum_{i=1}^{\infty} \mu\left(E \cap E_{i}\right) e_{i},
$$

where $e_{i}$ is the canonical basis of $c_{0}$.

Let $\mu_{i}=\left.\mu\right|_{E_{i}}$ be the restriction of $\mu$ to the subset $E_{i}$. We will denote by $\oplus_{c_{0}} L^{2}\left(\mu_{i}\right)$ the space of (classes of $\mu$-a.e. equal) measurable functions $f$ such that

(1) $f \chi_{E_{i}} \in L^{2}\left(\mu_{i}\right)$, and

(2) $\left(\left\|f \chi_{E_{i}}\right\|_{L^{2}\left(\mu_{i}\right)}\right)_{i=1}^{\infty} \in c_{0}$.

The (lattice) norm for this space is given by

$$
\|f\|_{\oplus_{c_{0}} L^{2}\left(\mu_{i}\right)}:=\sup _{i}\left\|f \chi_{E_{i}}\right\|_{L^{2}\left(\mu_{i}\right)}, \quad f \in \oplus_{c_{0}} L^{2}\left(\mu_{i}\right) .
$$

The following result shows that we can identify the spaces $L^{2}(\mathbf{n})$ and $\oplus_{c_{0}} L^{2}\left(\mu_{i}\right)$; for related examples, see [35, Example 4], [48, Example 8], [49, Example 6.47], and [59, Example 10].

Proposition 3.3.1. The natural identification map between $L^{2}(\mathbf{n})$ and $\oplus_{c_{0}} L^{2}\left(\mu_{i}\right)$ is an order isometry.

Proof. Let $f \in L^{2}(\mathbf{n})$. Then $f^{2} \in L^{1}(\mathbf{n})$ and so $f^{2} \chi_{E_{i}} \in L^{1}\left(\mu_{i}\right)$ and $\int f^{2} d \mathbf{n}=$ $\left(\int_{E_{i}} f^{2} d \mu_{i}\right)_{i} \in c_{0}$. That is, $f \chi_{E_{i}} \in L^{2}\left(\mu_{i}\right)$ and $\left(\left\|f \chi_{E_{i}}\right\|_{L^{2}\left(\mu_{i}\right)}\right)_{i} \in c_{0}$ since $\int_{E_{i}} f^{2} d \mu_{i}=\left\|f \chi_{E_{i}}\right\|_{L^{2}\left(\mu_{i}\right)}^{2}$. Hence, $f \in \oplus_{c_{0}} L^{2}\left(\mu_{i}\right)$ with

$$
\|f\|_{\oplus_{c_{0}} L^{2}\left(\mu_{i}\right)}^{2}=\sup _{i}\left\|f \chi_{E_{i}}\right\|_{L^{2}\left(\mu_{i}\right)}^{2}=\left\|\int f^{2} d \mathbf{n}\right\|_{c_{0}}=\left\|f^{2}\right\|_{L^{1}(\mathbf{n})}=\|f\|_{L^{2}(\mathbf{n})}^{2} .
$$


Therefore, $L^{2}(\mathbf{n}) \subset \oplus_{c_{0}} L^{2}\left(\mu_{i}\right)$ and the containment is an isometry.

Let now $f \in \oplus_{c_{0}} L^{2}\left(\mu_{i}\right)$. For every $x^{\prime}=\left\{\lambda_{i}\right\}_{i} \in \ell^{1}=c_{0}^{\prime}$ we have that $\left\langle\mathbf{n}, x^{\prime}\right\rangle(A)=\sum_{i} \lambda_{i} \mu\left(A \cap E_{i}\right)$. Then, $\left\langle\mathbf{n}, x^{\prime}\right\rangle=\lambda_{i} \mu_{i}$ and $\left|\left\langle\mathbf{n}, x^{\prime}\right\rangle\right|=\left|\lambda_{i}\right| \mu_{i}$ on $E_{i}$. Hence,

$$
\begin{gathered}
\int f^{2} d\left|\left\langle\mathbf{n}, x^{\prime}\right\rangle\right|=\sum_{i} \int_{E_{i}} f^{2} d\left|\left\langle\mathbf{n}, x^{\prime}\right\rangle\right|=\sum_{i}\left|\lambda_{i}\right| \int_{E_{i}} f^{2} d \mu_{i} \\
\leq\left\|x^{\prime}\right\|_{\ell^{1} \sup _{i}}\left\|f_{i} \chi_{E_{i}}\right\|_{L^{2}\left(\mu_{i}\right)}^{2}=\left\|x^{\prime}\right\|_{\ell^{1}}\|f\|_{\oplus_{c_{0}} L^{2}\left(\mu_{i}\right)}^{2}<\infty
\end{gathered}
$$

and so $f^{2} \in L^{1}\left(\left\langle\mathbf{n}, x^{\prime}\right\rangle\right)$. Moreover, $\left\{\int_{A \cap E_{i}} f^{2} d \mu_{i}\right\}_{i} \in c_{0}$ is such that

$$
\begin{gathered}
\left\langle x^{\prime},\left(\int_{A \cap E_{i}} f^{2} d \mu_{i}\right)_{i}\right\rangle=\sum_{i} \lambda_{i} \int_{A \cap E_{i}} f^{2} d \mu_{i}= \\
\sum_{i} \int_{A \cap E_{i}} f^{2} d\left\langle\mathbf{n}, x^{\prime}\right\rangle=\int_{A} f^{2} d\left\langle\mathbf{n}, x^{\prime}\right\rangle,
\end{gathered}
$$

so $f^{2} \in L^{1}(\mathbf{n})$ with $\int_{A} f^{2} d \mathbf{n}=\left\{\int_{A \cap E_{i}} f^{2} d \mu_{i}\right\}_{i}$. Then, $f \in L^{2}(\mathbf{n})$.

The proofs of the following corollaries are straightforward applications of Theorem 2.2.3, Theorem 3.2.2 with $M=L=\ell^{1}$ and Remark 3.2.3 (1).

Corollary 3.3.2. Let $X(\mu)=\oplus_{c_{0}} L^{2}\left(\mu_{i}\right)$ be the $c_{0}-$ sum of the spaces $L^{2}\left(\mu_{i}\right)$, $i \in \mathbb{N}$. Let $\left\{a_{i}\right\}_{i} \in \ell^{2}$, and assume that there exists an element $x^{\prime} \in\left(\ell^{1}\right)^{+}$such that $\left\{f_{i}\right\}_{i=1}^{\infty}$ is orthogonal with respect to $\left\langle\mathbf{n}, x^{\prime}\right\rangle$. If $\left\|\left(\left\langle\int f_{i}^{2} d \mathbf{n}, x^{\prime}\right\rangle\right)_{i=1}^{\infty}\right\|_{\ell^{1}}<\infty$, then $\sum_{i=1}^{\infty} a_{i} f_{i}$ converges $\left\langle\mathbf{n}, x^{\prime}\right\rangle$-a.e.

Corollary 3.3.3. Let $X(\mu)=\oplus_{c_{0}} L^{2}\left(\mu_{i}\right)$ be the $c_{0}-$ sum of the spaces $L^{2}\left(\mu_{i}\right)$, $i \in \mathbb{N}$. Let $\left(\left\{a_{i}\right\}_{i} \in \ell^{2}\right.$, and assume that there is a sequence of positive real numbers $\Delta=\left\{\varepsilon_{i}\right\} \in\left(\ell^{1}\right)^{+}$satisfying the inequalities in (1) of Theorem 2.2.3 for the vector measure $\mathbf{n}$. Then there is a sequence $0 \leq x^{\prime} \in B_{\ell^{1}}$ such that $\left\{f_{i}\right\}_{i}$ is orthogonal with respect to $\left\langle\mathbf{n}, x^{\prime}\right\rangle$ and $\sum_{i=1}^{\infty} a_{i} f_{i}$ converges $\left\langle\mathbf{n}, x^{\prime}\right\rangle$-a.e.

Let us finish the section with a particular example of a sequence that satisfies these corollaries.

Example 3.3.4. Let $([0,1], \Sigma, \mu)$ be the Lebesgue measure space. We consider the following partition of the interval $[0,1]$.

$$
E_{1}=\left[0, \frac{1}{2}\right], E_{2}=\left[\frac{1}{2}, \frac{3}{4}\right], E_{3}=\left[\frac{3}{4}, \frac{7}{8}\right], \ldots, E_{n}=\left[\frac{2^{n-1}-1}{2^{n-1}}, \frac{2^{n}-1}{2^{n}}\right], \ldots .
$$


For each $i=1,2, \ldots$, take a $\left.\mu\right|_{E_{i}}$-orthogonal sequence $\left(g_{i}^{j}\right)_{j=1}^{\infty}$ satisfying

$$
\int g_{i}^{j} g_{i}^{k} d \mu_{i}=\left\{\begin{array}{c}
0 \text { if } \quad k \neq j \\
2 \text { if } k=j
\end{array}\right.
$$

Now we define for each $n \in \mathbb{N}$ the function $f_{n}$ by $f_{n}:=\sum_{k=1}^{\infty} \lambda_{k}^{n} g_{k}^{n} \chi_{E_{k}}$, where the scalar numbers $\lambda_{k}^{n}$ are given by

$$
\lambda_{k}^{n}=\left\{\begin{array}{lll}
\frac{1}{2^{\frac{n-k+1}{2}}} & \text { if } & k<n \\
\frac{2^{\frac{k-n+1}{2}}}{2} & \text { if } & k \geq n .
\end{array}\right.
$$

Note that $f_{n} \chi_{E_{i}}=\lambda_{i}^{n} g_{i}^{n} \chi_{E_{i}} \in L^{2}\left(\mu_{i}\right)$ and $\left\|f_{n} \chi_{E_{i}}\right\|_{L^{2}\left(\mu_{i}\right)}=\sqrt{2}\left|\lambda_{i}^{n}\right| \rightarrow 0$ when $i \rightarrow \infty$, so $f \in \oplus_{c_{0}} L^{2}\left(\mu_{i}\right)=L^{2}(\mathbf{n})$. Let $\alpha:=\left\{\alpha_{k}\right\}_{k=1}^{\infty}=\left\{\frac{1}{2^{k}}\right\}_{k=1}^{\infty} \in B_{\ell^{1}}$. It is easy to see that the sequence $\left\{f_{n}\right\}_{n}$ is orthogonal with respect $\langle\mathbf{n}, \alpha\rangle$ for any $\alpha \in\left(\ell^{1}\right)_{\mathbf{n}}^{+}$. Taking $\alpha=\left\{\frac{1}{2^{k}}\right\}_{k}$, we have that

$$
\begin{gathered}
\sum_{n \geq 1} \int f_{n}^{2} d\langle\mathbf{n}, \alpha\rangle=\sum_{n \geq 1}\left\langle\int f_{n}^{2} d \mathbf{n}, \alpha\right\rangle=\sum_{n \geq 1} \sum_{k \geq 1} \frac{1}{2^{k}} \int_{E_{k}} f_{n}^{2} d \mu_{k} \\
=\sum_{n \geq 1} \sum_{k \geq 1} \frac{\left(\lambda_{k}^{n}\right)^{2}}{2^{k}} \int_{E_{k}}\left(g_{k}^{n}\right)^{2} d \mu_{k}=\sum_{n \geq 1} \sum_{k \geq 1} \frac{\left(\lambda_{k}^{n}\right)^{2}}{2^{k-1}} \\
=\sum_{n \geq 1} \sum_{k=1}^{n-1} \frac{1}{2^{n}}+\sum_{n \geq 1} \sum_{k \geq n} \frac{2^{n}}{2^{2 k}}<\infty,
\end{gathered}
$$

Thus, $\left\|\left(\left\langle\int f_{n}^{2} d \mathbf{n}, \alpha\right\rangle\right)\right\|_{\ell^{1}}$ is bounded. Remark 3.2.3 (1) provides the required condition on $\left\{a_{i}\right\}_{i}$ and so $\sum_{i=1}^{\infty} a_{i} f_{i}$ converges $\langle\mathbf{n}, \alpha\rangle$-a.e. 


\section{Chapter 4}

\section{Pointwise dependent Fourier coefficients}

This chapter is devoted to show a natural application of the vector measure orthogonality in the setting of function aproximation.

Throughout all the section $\mathbf{m}: \Sigma \rightarrow X$ will be a Banach space valued countably additive vector measure. Let $g \in L^{2}(\mathbf{m})$. We develop in this section a formalism to find an approximation to $g$ with respect to a distance defined using the norm $\|\cdot\|_{L^{2}(\mathbf{m})}$ by means of products of special sequences of functions $\left\{f_{i}\right\}_{i=1}^{\infty} \subset L^{2}(\mathbf{m})$ and other functions $\alpha_{i}^{\phi}, i \in \mathbb{N}$, whose definition depends on a Bochner integrable function $\phi$. Essentially the Bochner integrable function provides a parametrization of a family of measures that are defined as $\left\langle\mathbf{m}, x^{\prime}\right\rangle$ for different $x^{\prime} \in X^{\prime}$. The functions $\alpha_{i}^{\phi}$ will be called pointwise dependent Fourier coefficients, since the construction is closely related to the usual way to define these coefficients in the Hilbert space formalism. However, the series defined in this way is not given by a linear combination of the elements of an orthonormal system, since in our case the coefficients of the expansion are also functions. Thus, we obtain a non linear approximation to the function $g$. We establish our functional setting and we obtain our main approximation result (Theorem 4.1.7) giving some examples too. We also present in this section the explicit 
formula for the pointwise dependent Fourier coefficients for the case that the (parametric) measure is defined by a simple function. The easy representation of the coefficients that is obtained motivates the results in this section, where continuity of the pointwise dependent coefficients with respect to the Bochner norm is analyzed.

Let us introduce first some concepts and notation that will be needed in this chapter. Let $(\Omega, \Sigma, \mu)$ be a finite measure space, and let $f: \Omega \rightarrow X$ be a vector valued function, where $X$ is a Banach space. We say that $f$ is strongly $\mu$-measurable if there exists a sequence $\left\{\varphi_{n}\right\}_{n}$ of simple functions such that $\lim _{n \rightarrow \infty}\left\|f(\omega)-\varphi_{n}(\omega)\right\|_{X}=0$ for $\mu$-almost everywhere $\omega \in \Omega$. The collection of all strongly measurable functions from $\Omega$ to $X$ is denoted by $\mathcal{M}(\Omega, X)$. A strongly $\mu$-measurable function $f$ is Bochner integrable if there is a sequence $\left\{\varphi_{n}\right\}_{n}$ of simple functions such that the real measurable function $\left\|f-\varphi_{n}\right\|_{X}$ is Lebesgue integrable for each $n$ and $\lim _{n} \int\left\|f-\varphi_{n}\right\|_{X} d \mu=0$. The collection of all Bochner integrable functions $L^{1}(\mu, X)$ is a vector subspace of $\mathcal{M}(\Omega, X)$ and the Bochner integral acts as a linear operator from this space into $X$. Now let $\mu$ be a control measure for $\mathbf{m}$ that satisfies that $L^{1}(\mathbf{m}) \subseteq L^{1}(\mu)$. Such a measure always exists and it can be written as what is called Rybakov measure for $\mathbf{m}$, i.e. a measure $\left|\left\langle\mathbf{m}, x_{0}^{\prime}\right\rangle\right|$ for a certain $x_{0}^{\prime} \in X^{\prime}$ (see [25]). Since each element $x^{\prime} \in X^{\prime}$ defines a scalar measure we can consider Bochner integrable functions $\phi$ of the space $\mathcal{L}^{1}\left(\mu, X^{\prime}\right)$ that satisfy that $\langle\mathbf{m}, \phi(\omega)\rangle$ is a positive measure for each $\omega \in \Omega \mu$-a.e. The notion of $\mathbf{m}$-orthogonality for sequences of functions becomes useful to assure the orthogonality of the sequence with respect to every scalar product defined by each positive measure $\langle\mathbf{m}, \phi(\omega)\rangle, \omega \in \Omega$. In fact, this leads to the definition of the pointwise dependent Fourier coefficients, that are functions $\alpha_{i}: \Omega \rightarrow \mathbb{R}$.

\subsection{Pointwise dependent Fourier coefficients}

In this section we establish the main results concerning approximation of functions. Roughly speaking, we provide the mathematical tools to define parametric measures by means of Bochner integrable functions, which leads to the defini- 
tion of the pointwise dependent Fourier coefficients and motivates the definition of the adequate error for our technique. We also compute the coefficients when the Bochner integrable function that defines the parametric measure is a simple function. This leads to show how we can compute these coefficients in the general case by approximating by the ones that hold in the simple functions case. Throughout this section $(\Omega, \Sigma, \mu)$ will be a finite measure space that controls the Banach space valued measure $\mathbf{m}: \Sigma \rightarrow X$ and $L^{1}(\mathbf{m}) \subseteq L^{1}(\mu)$.

Let $1 \leq p<\infty$. Let $\phi: \Omega \rightarrow X$ be a Bochner integrable function. We use such kind of individual functions for defining the class of measures that will be considered in the following sections. We will considered also the space $L^{p}(\mu, X)$ of classes of Bochner p-integrable functions, that is, functions $\phi \in \mathcal{M}(\mu, X)$ such that $\int_{\Omega}\|\phi(\omega)\|_{X}^{p} d \mu(\omega)<\infty$. The norm of this space is given by

$$
\|\phi\|:=\left(\int_{\Omega}\|\phi(\omega)\|_{X}^{p} d \mu(\omega)\right)^{\frac{1}{p}}, \quad \phi \in L^{p}(\mu, X) .
$$

Although the functions $\phi$ as above will be considered as single functions, for the approximation results we will use the Bochner norm, so the functions $\phi$ will be considered sometimes as elements of $L^{p}(\mu, X)$, i.e as a representative of class of functions. For the aim of clarity, we will use sometimes the notation $[\phi]$ to denote the class of $\phi$. The set of (classes of) simple functions is dense in $L^{p}(\mu, X)$ (see for instance Definition 11.42 in [3, Ch.11]).

Definition 4.1.1. Let $X$ be a Banach space and $X^{\prime}$ its dual space. We say that a Bochner integrable function $\phi \in \mathcal{L}^{1}\left(\mu, X^{\prime}\right)$ defines a parametric measure $\mathbf{m}_{\phi(\omega)}$ if the formula

$$
\mathbf{m}_{\phi(\omega)}(A):=\langle\mathbf{m}, \phi(\omega)\rangle(A), \quad A \in \Sigma
$$

gives a positive measure that controls $\mu$ for every $\omega \in \Omega$.

Note that for a function $h \in L^{1}(\mathbf{m})$, the function $w \mapsto \int_{\Omega} h d \mathbf{m}_{\phi(\omega)}=$ $\left\langle\int h d \mathbf{m}, \phi(\omega)\right\rangle$ is measurable, since by hypothesis $\phi$ is strongly (and then weak ${ }^{*}$ ) measurable and $\int h d \mathbf{m} \in X$. This implies in particular that the functions $\alpha_{i}^{\phi}$ that appear in the Definition 4.1.2 are measurable. For the Definition 4.1.2 and some purposes of this section it is enough to assume that the function $\phi$ is Gel'fand integrable (see for instance $[3,11.9]$ ). However, for the approximation procedure that is developed the Bochner integrability is needed. 
Definition 4.1.2. Consider a $\mathbf{m}$-orthogonal sequence $\left\{f_{i}\right\}_{i=1}^{\infty}$ and a Bochner integrable function $\phi$ that defines the parametric measure $\mathbf{m}_{\phi(\omega)}$. For each $i \in N$, we define the $i$-th pointwise dependent Fourier coefficient of a function $g \in L^{2}(\mathbf{m})$ as the function $\alpha_{i}^{\phi}: \Omega \rightarrow \mathbb{R}$ given by

$$
\alpha_{i}^{\phi}(\omega):=\frac{\int_{\Omega} g f_{i} d \mathbf{m}_{\phi(\omega)}}{\int_{\Omega} f_{i}^{2} d \mathbf{m}_{\phi(\omega)}} .
$$

Definition 4.1.2 makes sense $\mu$-a.e.; in fact, if we have two different Bochner integrable functions $\phi_{1}$ and $\phi_{2}$ that belong to the same class of $\mathcal{L}^{1}\left(\mu, X^{\prime}\right)$, the coefficients $\alpha_{i}^{\phi_{1}}(\omega)$ and $\alpha_{i}^{\phi_{2}}(\omega)$ are equal $\mu$-a.e. However, note that these functions are defined pointwise, and will be considered in this way through all the section.

Definition 4.1.3. Let $\phi \rightarrow X^{\prime}$ be a Bochner integrable function. We say that a sequence of measurable functions $\left\{\beta_{i}\right\}_{i=1}^{\infty}, \beta_{i}: \Omega \rightarrow \mathbb{R}$, is $\phi$-compatible with the $\mathbf{m}$-orthogonal sequence $\left\{f_{i}\right\}_{i=1}^{\infty}$ (compatible for short if the function $\phi$ is clear in the context) if the function $\omega \mapsto \sum_{i=1}^{\infty} \beta_{i}^{2}(\omega)\left(\int_{\Omega} f_{i}^{2} d \mathbf{m}_{\phi(\omega)}\right)$ is integrable with respect to $\mu$.

Lemma 4.1.4. Let $\phi: \Omega \rightarrow X^{\prime}$ be a Bochner integrable function that defines a parametric measure $\mathbf{m}_{\phi(\omega)}$. Let $\left\{f_{i}\right\}_{i=1}^{\infty}$ be a $\mathbf{m}$-orthogonal sequence and consider a function $g \in L^{2}(\mathbf{m})$. Then the corresponding sequence of pointwise dependent Fourier coefficients $\left\{\alpha_{i}^{\phi}\right\}_{i=1}^{\infty}$ is $\phi$-compatible with $\left\{f_{i}\right\}_{i=1}^{\infty}$.

Proof. Each function $\alpha_{i}^{\phi}$ is measurable since it is defined as a quotient of measurable functions. Recall that $g \in L^{2}\left(\mathbf{m}_{\phi(\omega)}\right)$ for every $\omega \in \Omega$. Clearly for every $\omega \in \Omega, \sum_{i=1}^{\infty} \alpha_{i}^{\phi}(\omega) f_{i}(\eta)$ is integrable with respect to the measure $\mathbf{m}_{\phi(\omega)}$ as a consequence of the definition of the functions $\alpha_{i}^{\phi}$ and the Hilbert space structure of $L^{2}\left(\mathbf{m}_{\phi(\omega)}\right)$ at each point $\omega$. Fix such an $\omega \in \Omega$. Bessel's inequality gives

$$
\sum_{i=1}^{\infty}\left|\alpha_{i}^{\phi}(\omega)\right|^{2} \int_{\Omega} f_{i}^{2} d \mathbf{m}_{\phi(\omega)}=\sum_{i=1}^{\infty} \frac{\left(\int_{\Omega} g f_{i} d \mathbf{m}_{\phi(\omega)}\right)^{2}}{\int_{\Omega} f_{i}^{2} d \mathbf{m}_{\phi(\omega)}} \leq\|g\|_{L^{2}\left(\mathbf{m}_{\phi(\omega)}\right)}^{2} .
$$

The function $\omega \mapsto\|g\|_{L^{2}\left(\mathbf{m}_{\phi(\omega)}\right)}^{2}$ is measurable. Thus, the computation

$$
\begin{gathered}
\|\| g\left\|_{L^{2}\left(\mathbf{m}_{\phi(\omega)}\right)}^{2}\right\|_{L^{1}(\mu)}=\int_{\Omega}\left|\int_{\Omega} g^{2}(\eta) d \mathbf{m}_{\phi(\omega)}(\eta)\right| d \mu(\omega) \\
=\int_{\Omega}\left\langle\int_{\Omega} g^{2}(\eta) d \mathbf{m}(\eta), \phi(\omega)\right\rangle d \mu(\omega)
\end{gathered}
$$




$$
\leq\left\|\int_{\Omega} g^{2}(\eta) d \mathbf{m}(\eta)\right\| \cdot \int_{\Omega}\|\phi(\omega)\| d \mu(\omega)=\|g\|_{L^{2}(\mathbf{m})}^{2} \cdot\|\phi\|_{L^{1}\left(\mu, X^{\prime}\right)}<\infty
$$

finishes the proof.

Lemma 4.1.5. Let $\phi: \Omega \rightarrow X^{\prime}$ be a Bochner integrable function. Let $\left\{\beta_{i}\right\}_{i=1}^{\infty}$ be a sequence of functions that is $\phi$-compatible with the $\mathbf{m}$-orthogonal sequence $\left\{f_{i}\right\}_{i=1}^{\infty}$ and let $g \in L^{2}(\mathbf{m})$. Then the function

$$
\psi(\omega):=\int_{\Omega}\left(g(\eta)-\sum_{i=1}^{\infty} \beta_{i}(\omega) f_{i}(\eta)\right)^{2} d \mathbf{m}_{\phi(\omega)}(\eta)
$$

is defined $\mu$-a.e and integrable with respect to $\mu$.

Proof. By the definition of compability we have that

$$
h(\omega)=\sum_{i=1}^{\infty} \beta_{i}(\omega)^{2} \int f_{i}^{2} d \mathbf{m}_{\phi(\omega)} \in L^{1}(\mu) .
$$

Then, $h(\omega)<\infty$ for $\mu$-a.e. $\omega \in \Omega$ and so $\sum_{i=1}^{\infty} \beta_{i}(\omega) f_{i} \in L^{2}\left(\mathbf{m}_{\phi(\omega)}\right) \mu$-a.e. $\omega \in \Omega$. Hence, $\psi$ is well defined $\mu$-a.e. and

$$
\begin{gathered}
\psi(\omega)=\left\|g-\sum_{i=1}^{\infty} \beta_{i}(\omega) f_{i}\right\|_{L^{2}\left(\mathbf{m}_{\phi(\omega)}\right)}^{2} \\
=\int g^{2} d \mathbf{m}_{\phi(\omega)}+\sum_{i=1}^{\infty} \beta_{i}(\omega)^{2} \int f_{i}^{2} d \mathbf{m}_{\phi(\omega)}-2 \sum_{i=1}^{\infty} \beta_{i}(\omega) \int g f_{i} d \mathbf{m}_{\phi(\omega)}
\end{gathered}
$$

is measurable. Moreover,

$$
\psi(\omega)^{1 / 2} \leq\|g\|_{L^{2}\left(\mathbf{m}_{\phi(\omega)}\right)}+\left\|\sum_{i=1}^{\infty} \beta_{i}(\omega) f_{i}\right\|_{L^{2}\left(\mathbf{m}_{\phi(\omega)}\right)} \in L^{2}(\mu)
$$

since $\omega \rightarrow\|g\|_{L^{2}\left(\mathbf{m}_{\phi(\omega)}\right)}^{2}$ is in $L^{1}(\mu)$ as $\phi \in \mathcal{L}^{1}\left(\mu, X^{\prime}\right)$ (see the proof of Lemma 4.1.4) and $\omega \rightarrow\left\|\sum_{i=1}^{\infty} \beta_{i}(\omega) f_{i}\right\|_{L^{2}\left(\mathbf{m}_{\phi(\omega)}\right)}^{2}=h(\omega) \in L^{1}(\mu)$. Then, $\psi \in L^{1}(\mu)$.

Lemma 4.1.5 allows us to give the following definition.

Definition 4.1.6. Let $\phi \in \mathcal{L}^{1}\left(\mu, X^{\prime}\right)$ be a function that defines a parametric measure $\mathbf{m}_{\phi(\omega)}$ and let $\left\{f_{i}\right\}_{i=1}^{\infty}$ be a $\mathbf{m}$-orthogonal sequence. Let $g \in L^{2}(\mathbf{m})$ and consider a sequence of functions $\left\{\beta_{i}\right\}_{i=1}^{\infty}$ which is compatible with $\left\{f_{i}\right\}_{i=1}^{\infty}$. We define the error $\epsilon$ associated to the function $g \in L^{2}(\mathbf{m})$ and the sequence $\left\{\beta_{i}\right\}_{i=1}^{\infty}$ by

$$
\epsilon\left(g,\left\{\beta_{i}\right\}_{i=1}^{\infty}\right):=\int_{\Omega}\left(\int_{\Omega}\left(g(\eta)-\sum_{i=1}^{\infty} \beta_{i}(\omega) f_{i}(\eta)\right)^{2} d \mathbf{m}_{\phi(\omega)}(\eta)\right) d \mu(\omega) .
$$


The following result is a direct consequence of the definitions and Lemma 4.1.5 and relates the best approximation with respect to the error $\epsilon$ to the sequence of pointwise dependent Fourier coefficients.

Theorem 4.1.7. Let $\phi \in \mathcal{L}^{1}\left(\mu, X^{\prime}\right)$ be a function that defines a parametric measure $\mathbf{m}_{\phi(\omega)}$ and let $g \in L^{2}(\mathbf{m})$. Let $\left\{f_{i}\right\}_{i=1}^{\infty}$ be a $\mathbf{m}$-orthogonal sequence. If $\left\{\beta_{i}\right\}_{i=1}^{\infty}$ is a sequence of functions that is $\phi$-compatible with $\left\{f_{i}\right\}_{i=1}^{\infty}$, then

$$
\epsilon\left(g,\left\{\alpha_{i}^{\phi}\right\}_{i=1}^{\infty}\right) \leq \epsilon\left(g,\left\{\beta_{i}\right\}_{i=1}^{\infty}\right) .
$$

Moreover, $\epsilon\left(g,\left\{\alpha_{i}^{\phi}\right\}_{i=1}^{\infty}\right)=\epsilon\left(g,\left\{\beta_{i}\right\}_{i=1}^{\infty}\right)$ only in the case that $\alpha_{i}^{\phi}=\beta_{i} \mu$-a.e.

Proof. Consider the functions

$$
\psi_{\alpha}(\omega):=\left(\int_{\Omega}\left(\sum_{i=1}^{\infty} \alpha_{i}^{\phi}(\omega) f_{i}(\eta)\right)^{2} d \mathbf{m}_{\phi(\omega)}(\eta)\right)^{\frac{1}{2}}
$$

and

$$
\psi_{\beta}(\omega):=\left(\int_{\Omega}\left(\sum_{i=1}^{\infty} \beta_{i}(\omega) f_{i}(\eta)\right)^{2} d \mathbf{m}_{\phi(\omega)}(\eta)\right)^{\frac{1}{2}}
$$

and take a $\mu$-null set $A$ satisfying that $\psi_{\alpha}$ and $\psi_{\beta}$ are defined for the points $\omega \in \Omega \backslash A$. Then, since $g \in L^{2}(\mathbf{m})$, we have that $g \in L^{2}\left(\mathbf{m}_{\phi(\omega)}\right)$ for every $\omega \in \Omega \backslash A$. The Hilbert space structure of the spaces $L^{2}\left(\mathbf{m}_{\phi(\omega)}\right)$ gives the inequalities

$$
\int_{\Omega}\left(g-\sum_{i=1}^{\infty} \alpha_{i}^{\phi}(\omega) f_{i}(\eta)\right)^{2} d \mathbf{m}_{\phi(\omega)}(\eta) \leq\left(\int_{\Omega}\left(g-\sum_{i=1}^{\infty} \beta_{i}(\omega) f_{i}(\eta)\right)^{2} d \mathbf{m}_{\phi(\omega)}(\eta)\right)
$$

for each point $\omega \in \Omega \backslash A$, since the pointwise dependent Fourier coefficients gives the best approximation at such $\omega$ in each space $L^{2}\left(\mathbf{m}_{\phi(\omega)}\right)$. Then the result is a direct consequence of the lattice properties of $L^{2}(\mu)$ (see Section 1 of [42]). The fact the equality $\epsilon\left(g,\left\{\alpha_{i}\right\}_{i=1}^{\infty}\right)=\epsilon\left(g,\left\{\beta_{i}\right\}_{i=1}^{\infty}\right)$ holds only in the case when $\alpha_{i}^{\phi}=\beta_{i} \mu-a . e$. is a direct consequence of the above proof.

Let $\phi_{1}$ and $\phi_{2}$ be Bochner integrable functions that define parametric measures $\mathbf{m}_{\phi_{1}}$ and $\mathbf{m}_{\phi_{2}}$. Suppose that they satisfy that there is a positive function $k(\omega)$ such that $\phi_{1}(\omega)=k(\omega) \phi_{2}(\omega) \mu$-a.e.. Then it is clear by the definition of the pointwise dependent Fourier coefficients that we obtain the equality

$$
\alpha_{i}^{\phi_{1}}(\omega)=\alpha_{i}^{\phi_{2}}(\omega)
$$

for every $\omega \mu$-a.e. This motivates the following definition. 
Definition 4.1.8. We say that a strongly measurable function $\phi: \Omega \rightarrow X$ is pointwise normalized if $\|\phi(\omega)\|_{X}=1$ for every $\omega \in \Omega$. We will write $C(\mu, X)$ for the set of all (classes of) these functions. Note that $C(\mu, X)$ is included in all spaces $L^{p}(\mu, X), 1 \leq p \leq \infty$.

Proposition 4.1.9. Let $1 \leq p<\infty$ and let $X$ be a Banach space. The set $C(\mu, X)$ is closed subset in $L^{p}(\mu, X)$, and the set of simple functions

$$
S C(\mu, X)=\left\{\sum_{i=1}^{m} v_{i} \chi_{A_{i}}:\left\|v_{i}\right\|_{X}=1,\left\{A_{i}\right\}_{i=1}^{m} \subset \Sigma \text { partition of } \Omega, m \in \mathbb{N}\right\}
$$

is dense in $C(\mu, X)$.

Proof. Consider a sequence $\left\{\phi_{n}\right\}_{n} \subset C(\mu, X)$ converging to $\phi$ in $L^{p}(\mu, X)$. Then,

$$
\begin{gathered}
\left\|\phi-\phi_{n}\right\|_{L^{p}(\mu, X)}^{p}=\int_{\Omega}\left\|\phi(\omega)-\phi_{n}(\omega)\right\|_{X}^{p} d \mu(\omega) \\
\geq \int_{\Omega}\left|\|\phi(\omega)\|_{X}-\left\|\phi_{n}(\omega)\right\|_{X}\right|^{p} d \mu(\omega) \\
=\int_{\Omega}\left|\|\phi(\omega)\|_{X}-1\right|^{p} d \mu(\omega)
\end{gathered}
$$

for all $n$ and so $\int_{\Omega}\left|\|\phi(\omega)\|_{X}-1\right|^{p} d \mu(\omega)=0$. Therefore, $\|\phi(\omega)\|_{X}=1 \mu$-a.e. $\omega \in \Omega$.

Now let us show that $S C(\mu, X)$ is dense in $C(\mu, X)$. Let $\phi$ be a function of $C(\mu, X)$. Since the set of all the simple functions is dense in $L^{p}(\mu, X)$, there is a sequence of simple functions $\left\{\phi_{n}\right\}_{n}$ converging to $\phi$ in $L^{p}(\mu, X)$. We can assume without loss of generality that $\left\|\phi_{n}(\omega)\right\| \neq 0$ for all $\omega \in \Omega$. Otherwise, we can take $\phi_{n}^{\prime}=\phi_{n}+\frac{x_{0}}{n} \chi_{A_{n}}$ where $x_{0}$ is a norm one element of $X$ and $A_{n}=$ $\left\{\omega \in \Omega:\left\|\phi_{n}(\omega)\right\|=0\right\}$, and so $\left\|\phi-\phi_{n}^{\prime}\right\|_{L^{p}(\mu, X)} \leq\left\|\phi-\phi_{n}\right\|_{L^{p}(\mu, X)}+\frac{1}{n} \mu(\Omega)^{1 / p}$. Taking $\psi_{n}(\omega)=\frac{\phi_{n}(\omega)}{\left\|\phi_{n}(\omega)\right\|_{X}} \in S C(\mu, X)$, we have that

$$
\begin{gathered}
\left\|\phi-\psi_{n}\right\|_{L^{p}(\mu, X)} \leq\left\|\phi-\phi_{n}\right\|_{L^{p}(\mu, X)}+\left(\int\left\|\phi_{n}(\omega)\left(1-\frac{1}{\left\|\phi_{n}(\omega)\right\|_{X}}\right)\right\|_{X}^{p} d \mu\right)^{1 / p} \\
=\left\|\phi-\phi_{n}\right\|_{L^{p}(\mu, X)}+\left(\int\left|\left\|\phi_{n}(\omega)\right\|_{X}-1\right|^{p} d \mu\right)^{1 / p} \\
\leq 2\left\|\phi-\phi_{n}\right\|_{L^{p}(\mu, X)} .
\end{gathered}
$$


We can obtain with a direct calculation the expression of the pointwise dependent Fourier coefficient when the function $\phi$ that defines the parametric measure $\mathbf{m}_{\phi(\omega)}$ is a simple function. If $\phi(\omega):=\sum_{i=1}^{m} x_{i}^{\prime} \chi_{A_{i}}(\omega)$ and $f_{j}$ is an element of the $\mathbf{m}$-orthogonal sequence $\left\{f_{i}\right\}_{i=1}^{\infty}$, we obtain

$$
\alpha_{j}^{\phi}(\omega)=\frac{\sum_{i=1}^{m}\left\langle\int_{\Omega} g f_{j} d \mathbf{m}, x_{i}^{\prime}\right\rangle \chi_{A_{i}}(\omega)}{\sum_{i=1}^{m}\left\langle\int_{\Omega} f_{j}^{2} d \mathbf{m}, x_{i}^{\prime}\right\rangle \chi_{A_{i}}(\omega)}=\sum_{i=1}^{m} \frac{\left\langle\int_{\Omega} g f_{j} d \mathbf{m}, x_{i}^{\prime}\right\rangle}{\left\langle\int_{\Omega} f_{j}^{2} d \mathbf{m}, x_{i}^{\prime}\right\rangle} \chi_{A_{i}}(\omega),
$$

where $\left\langle\int_{\Omega} f_{j}^{2} d \mathbf{m}, x_{i}^{\prime}\right\rangle \neq 0$ for every $i=1, \ldots, m$ since $\int f_{j}^{2} d \mathbf{m}_{\phi(\omega)}$ for all $\omega$ and $\left\{A_{i}\right\}_{i=1}^{m}$ is a disjoint sequence. In other case, the corresponding $i$-th term does not appear in the sum.

This shows that the calculus of the pointwise dependent Fourier coefficients is easy when $\phi$ is a simple function. On the other hand, Proposition 4.1.9 implies that we can approximate every normalized function that can be used to define a parametric measure by means of simple functions of the set $S C\left(\mu, X^{\prime}\right)$. Therefore, the construction of an approximation procedure depends on the definition of reasonable conditions to assure the continuity of the pointwise dependent Fourier coefficients $\alpha_{i}^{\phi}$ with respect to $\phi$ in $\mathcal{L}^{p}\left(\mu, X^{\prime}\right)$ for a certain $1 \leq p<\infty$. We will establish such conditions in the following section.

Example 4.1.10. (1) Let $([-\pi, \pi], \Sigma, \mu)$ be the Lebesgue measure space and consider the vector measure $\mathbf{m}_{0}: \Sigma \rightarrow \ell^{\infty}$ given by

$$
\mathbf{m}_{0}(A):=\left\{\int_{A \cap[-\pi, \pi]} \cos ^{2}(k x) d \mu\right\}_{k=1}^{\infty} .
$$

The measure $\mathbf{m}_{0}$ is clearly countably additive since each integral in $A$ in the definition is bounded by $\mu(A)$. Consider the functions $f_{1}(x)=\sin x$, $f_{2}(x)=\sin 2 x, \ldots, f_{n}(x)=\sin n x, n \geqslant 1$. Let us show that this family of functions defines an $\mathbf{m}_{\mathbf{0}}$ - orthogonal sequence. For every couple $n, m \in \mathbb{N}$ and $k \in \mathbb{N}$ such that the following expressions are defined, we obtain the following equalities.

a) If $m \neq n$, the integral of the product $f_{n} f_{m}$ and the corresponding weight is given by

$$
\int f_{n} f_{m} d \mathbf{m}_{0}=\left\{\int_{-\pi}^{\pi} \sin (n x) \sin (m x) \cos ^{2}(k x) d x\right\}_{k=1}^{\infty}
$$




$$
\begin{aligned}
& =\left\{\frac { 1 } { 8 } \left(\frac{\sin [(2 k-m-n) \pi]}{-2 k+m+n}+\frac{\sin [2(m-n) \pi]}{m-n}+\frac{\sin [(2 k+m-n) \pi]}{2 k+m-n}\right.\right. \\
& \left.\left.+\frac{\sin [(2 k-m+n) \pi]}{2 k-m+n}-\frac{2 \sin [(m+n) \pi]}{m+n}-\frac{\sin [(2 k+m+n) \pi]}{2 k+m+n}\right)\right\}_{k=1}^{\infty} .
\end{aligned}
$$

b) For $m=n$ and $k \neq n$, we get

$$
\begin{gathered}
\int f_{n} f_{n} d \mathbf{m}_{0}=\left\{\int_{-\pi}^{\pi} \sin ^{2}(n x) \cos ^{2}(k x) d x\right\}_{k=1}^{\infty} \\
=\left\{\frac { 1 } { 8 } \left(4 \pi+\frac{2 \sin (2 k \pi)}{k}-\frac{\sin (2(k-n) \pi)}{k-n}\right.\right. \\
\left.\left.-\frac{2 \sin (2 n \pi)}{n}-\frac{\sin (2(k+n)) \pi}{k+n}\right)\right\}_{k=1}^{\infty} .
\end{gathered}
$$

Notice that the quotients of the expressions above are 0 for some values $n, m, k$. Consider now the functions that are given for $i, j, r \in \mathbb{N}, k=2^{r}$, $n=3 i$ and $m=3 j$. Define the (countably additive) vector measure $\mathbf{n}_{0}(A):=\left\{\int_{A \cap[-\pi, \pi]} \frac{2}{\pi} \cos ^{2}\left(2^{r} x\right) d \mu\right\}_{r=1}^{\infty}$. Then, as a consequence of the calculations written above,

$$
\int f_{n} f_{m} d \mathbf{n}_{0}=\left\{\delta_{n m}\right\}_{r=1}^{\infty}
$$

Thus, for these values, the sequence $\left\{f_{3 i}\right\}_{i=1}^{\infty}, i \in \mathbb{N}$ defines an $\mathbf{n}_{0}$-orthogonal sequence.

(2) Let us define a parametric measure using the vector measure $\mathbf{n}_{0}$ given above. Consider the family of sets $I_{n}=\left[-\pi+\frac{\pi}{2^{n}}, \pi-\frac{\pi}{2^{n}}\right]$ and the basis $\left\{e_{n}\right\}_{n}$ of $\ell^{1}$. Let us take the Bochner integrable function $\phi$ given by

$$
\phi(\omega)=\sum_{n \geq 1} \chi_{I_{n} \backslash I_{n-1}}(\omega) e_{n}
$$

It is easy to proof that this function is strongly measurable that is the limit of the sequence of simple functions $\phi_{N}(\omega):=\sum_{n=1}^{N} \chi_{I_{n} \backslash I_{n-1}}(\omega) e_{n}$, $n \in \mathbb{N}$. Let $g(x)=x$ and compute the corresponding pointwise dependent Fourier coefficients. For every $i \in \mathbb{N}$,

$$
\begin{gathered}
\alpha_{i}^{\phi}(\omega)=\frac{\left\langle\int_{[-\pi, \pi]} g f_{i} d \mathbf{n}_{0}, \phi(\omega)\right\rangle}{\left\langle\int_{[-\pi, \pi]} f_{i}^{2} d \mathbf{n}_{0}, \phi(\omega)\right\rangle} \\
=\sum_{r \geq 1}\left(\frac{\cos \left(\left(2^{1+r}-3 i\right) \pi\right)}{2^{1+r}-3 i}-\frac{2 \cos (3 i \pi)}{3 i}-\frac{\cos \left(\left(2^{1+r}-3 i\right) \pi\right)}{2^{1+r}+3 i}\right) \chi_{\left[I_{r} \backslash I_{r-1}\right]}(\omega) .
\end{gathered}
$$

Therefore, the function $h(\omega):=\sum_{i=1}^{\infty} \alpha_{i}^{\phi}(\omega) \sin (3 i \omega)$ approximates $g$. 


\subsection{Continuity of the pointwise dependent Fourier coefficients}

Take a vector measure $\mathbf{m}$ and a Bocher integrable function $\phi$ and a sequence of simple functions $\left\{\phi_{n}\right\}_{n=1}^{\infty}$ whose classes converges to $[\phi]$ in the Bochner space $\mathcal{L}^{1}\left(\mu, X^{\prime}\right)$ such that each $\phi_{n}$ defines a parametric measure. In this point we analyze the convergence properties of the pointwise dependent Fourier coefficients $\alpha_{i}^{\phi_{n}}$ to $\alpha_{i}^{\phi}$ for a given $\mathbf{m}$-orthogonal sequence $\left\{f_{i}\right\}_{i=1}^{\infty}$ and a function $g \in L^{2}(\mathbf{m})$. Our aim is to show that under the adequate requirements on the functions $\phi_{n}$ and $\phi$, the projections $\sum_{i=1}^{\infty} \alpha_{i}^{\phi_{n}} f_{i}$ converge to $\sum_{i=1}^{\infty} \alpha_{i}^{\phi} f_{i}$ in $L^{2}(\mathbf{m})$. Further assumptions are necessary to obtain the results of this section. It is important to note that the results of the technique that we present are meaningful even in the case of finite (even small) sequences of $\mathbf{m}$-orthogonal functions, since the formulas provide a nonlinear approximation that is completely different to the usual (Hilbert space) linear approximations. We will show an easy example. We use that $L^{1}(\mathbf{m}) \subseteq L^{1}(\mu)$, so the $\mathbf{m}$-orthogonal sequence $\left\{f_{i}\right\}_{i=1}^{\infty}$ belongs to $L^{2}(\mu)$. For instance, this happens in the case when $\mu$ is defined as $\left\langle\mathbf{m}, x^{\prime}\right\rangle$ for a certain element $x^{\prime} \in X^{\prime}$. In general, we will assume that the vector measure $\mathbf{m}$ is equivalent to $\mu$.

We use a pointwise boundedness condition for the functions that define a parametric measure.

Definition 4.2.1. Let $\epsilon>0$. We say that a Bochner integrable function $\phi$ : $\Omega \rightarrow X^{\prime}$ that defines a parametric measure $\mathbf{m}_{\phi(\omega)}$ is $\epsilon$-lower bounded with respect to $\left\{f_{i}\right\}_{i=1}^{\infty}$ if for every $i \in \mathbb{N}$,

$$
\epsilon<\left\langle\int_{\Omega} f_{i}^{2} d \mathbf{m}, \phi(\omega)\right\rangle
$$

$\mu$-a.e.

Lemma 4.2.2. Let $g \in L^{2}(\mathbf{m})$ and let $\phi \in \mathcal{L}^{1}\left(\mu, X^{\prime}\right)$ be a Bochner integrable function that defines a parametric measure $\mathbf{m}_{\phi(\omega)}$ such that $\phi$ is $\epsilon$-lower bounded with respect to $\left\{f_{i}\right\}_{i=1}^{\infty}$. Then the pointwise dependent Fourier coefficient $\alpha_{i}^{\phi}$ of $g$ is square integrable with respect to $\mu$ for every $i \in \mathbb{N}$. Moreover, $\left\{\alpha_{i}^{\phi}\right\}_{i=1}^{\infty}$ is a norm bounded sequence of $L^{2}(\mu)$. 
4.2 Continuity of the pointwise dependent

Proof. Let $i \in \mathbb{N}$, then

$$
\begin{gathered}
\left(\alpha_{i}^{\phi}(\omega)\right)^{2}=\frac{\left(\int_{\Omega} g f_{i} d \mathbf{m}_{\phi(\omega)}\right)^{2}}{\left(\int_{\Omega} f_{i}^{2} d \mathbf{m}_{\phi(\omega)}\right)^{2}} \\
\leq \frac{\int_{\Omega} g^{2} d \mathbf{m}_{\phi(\omega)} \int_{\Omega} f_{i}^{2} d \mathbf{m}_{\phi(\omega)}}{\left(\int_{\Omega} f_{i}^{2} d \mathbf{m}_{\phi(\omega)}\right)^{2}} \leq \frac{\left\|\int_{\Omega} g^{2} d \mathbf{m}\right\|\|\phi(\omega)\|}{\epsilon} .
\end{gathered}
$$

Thus,

$$
\int_{\Omega}\left(\alpha_{i}^{\phi}(\omega)\right)^{2} d \mu \leq \frac{\left\|\int_{\Omega} g^{2} d \mathbf{m}\right\|}{\epsilon}\|\phi\|_{\mathcal{L}^{1}\left(\mu, X^{\prime}\right)} .
$$

This inequality and the properties of the Banach function space $L^{2}(\mu)$ give the result.

Theorem 4.2.3. Let $g \in L^{2}(\mathbf{m})$. Let $\epsilon>0$ and let $\phi \in C\left(\mu, X^{\prime}\right)$ be a function such that it defines a parametric measure $\mathbf{m}_{\phi(\omega)}$ and it is $\epsilon$-lower bounded with respect to $\left\{f_{i}\right\}_{i=1}^{\infty}$. Let $\left\{\phi_{n}\right\}_{n=1}^{\infty}$ be a sequence of functions of $S C\left(\mu, X^{\prime}\right)$ such that it defines a sequence of parametric measures $\mathbf{m}_{\phi_{n}(\omega)}$ and that converges to $\phi$ in $\mathcal{L}^{2}\left(\mu, X^{\prime}\right)$. Then for every $i \in \mathbb{N}$,

$$
\lim _{n \rightarrow \infty}\left\|\alpha_{i}^{\phi}-\alpha_{i}^{\phi_{n}}\right\|_{L^{2}(\mu)}=0 .
$$

Proof. Let $i \in \mathbb{N}$. Then there is a natural number $n_{0}$ such that for every $n \geq n_{0}$

$$
\epsilon<\left\langle\int_{\Omega} f_{i}^{2} d \mathbf{m}, \phi_{n}(\omega)\right\rangle
$$

$\mu$-a.e. We will simply write $\left\{\phi_{n}\right\}_{n=1}^{\infty}$ for the subsequence $\left\{\phi_{n}\right\}_{n=n_{0}}^{\infty}$. Since $\mu$ is a finite measure, an application of Lemma 4.2.2 gives that $\alpha_{i}^{\phi}, \alpha_{i}^{\phi_{n}} \in L^{2}(\mu)$ for each $n \in \mathbb{N}$. Now we define the function

$$
h_{n}(\omega):=\frac{\left\langle\int_{\Omega} f_{i}^{2} d \mathbf{m}, \phi(\omega)\right\rangle}{\left\langle\int_{\Omega} f_{i}^{2} d \mathbf{m}, \phi_{n}(\omega)\right\rangle}, \quad \omega \in \Omega,
$$

for every $n \in \mathbb{N}$. Then the result is a direct consequence of the following calculations.

$$
\begin{gathered}
\left\|\alpha_{i}^{\phi}-\alpha_{i}^{\phi_{n}}\right\|_{L^{2}(\mu)} \\
=\left(\int_{\Omega} \frac{\left(\left\langle\int_{\Omega} g f_{i} d \mathbf{m}, \phi(\omega)\right\rangle-h_{n}(\omega)\left\langle\int_{\Omega} g f_{i} d \mathbf{m}, \phi_{n}(\omega)\right\rangle\right)^{2}}{\left\langle\int_{\Omega} f_{i}^{2} d \mathbf{m}, \phi(\omega)\right\rangle^{2}} d \mu\right)^{\frac{1}{2}} \\
\leq\left\|\int_{\Omega} g f_{i} d \mathbf{m}\right\| \frac{1}{\epsilon}\left(\int_{\Omega}\left\|\phi-h_{n} \phi_{n}\right\|^{2} d \mu\right)^{\frac{1}{2}}
\end{gathered}
$$




$$
\begin{aligned}
& \leq\left\|\int_{\Omega} g f_{i} d \mathbf{m}\right\| \frac{1}{\epsilon}\left(\int_{\Omega}\left\|\phi-\phi_{n}\right\|^{2} d \mu\right)^{\frac{1}{2}}+\left\|\int_{\Omega} g f_{i} d \mathbf{m}\right\| \frac{1}{\epsilon}\left(\int_{\Omega}\left(1-h_{n}\right)^{2}\left\|\phi_{n}\right\|^{2} d \mu\right)^{\frac{1}{2}} \\
& \leq\left\|\int_{\Omega} g f_{i} d \mathbf{m}\right\| \frac{1}{\epsilon}\left[\left(\int_{\Omega}\left\|\phi-\phi_{n}\right\|^{2} d \mu\right)^{\frac{1}{2}}+\frac{1}{\epsilon}\left\|\int_{\Omega} f_{i}^{2} d \mathbf{m}\right\|\left(\int_{\Omega}\left\|\phi-\phi_{n}\right\|^{2} d \mu\right)^{\frac{1}{2}}\right] .
\end{aligned}
$$

The previous results show that the set of simple functions is dense in $C\left(\mu, X^{\prime}\right)$ in all spaces $\mathcal{L}^{p}\left(\mu, X^{\prime}\right)$. Therefore, Theorem 4.2.3 implies that under the assumption that $\phi$ is $\epsilon$-lower bounded with respect to $\left\{f_{i}\right\}_{i=1}^{\infty}$, we can approximate each Fourier coefficient $\alpha_{i}^{\phi}(i \in \mathbb{N})$ by the sequence $\left\{\alpha_{i}^{\phi_{n}}\right\}_{n=1}^{\infty}$ whenever $\left\{\phi_{n}\right\}_{n=1}^{\infty}$ is a sequence of simple functions defining a sequence of parametric measures $\mathbf{m}_{\phi_{n}(\omega)}$ and converging to $\phi$ in $\mathcal{L}^{2}\left(\mu, X^{\prime}\right)$. In the case that the $\mathbf{m}$-orthogonal sequence is finite we obtain an approximation calculus under the assumptions given above.

Definition 4.2.4. Let $m \in \mathbb{N}$ and consider a finite $\mathbf{m}$-orthogonal sequence $\left\{f_{i}\right\}_{i=1}^{m}$. Let $\phi \in C\left(\mu, X^{\prime}\right)$ be an $\varepsilon$-lower bounded that defines a parametric measure. Then we define the 1-error for the approximation of a function $g \in$ $L^{2}(\mathbf{m})$ by

$$
E_{1}(g, \phi):=\left\|g-\sum_{i=1}^{m} \alpha_{i}^{\phi} f_{i}\right\|_{L^{1}(\mu)} .
$$

Corollary 4.2.5. Let $m \in \mathbb{N}$ and consider a function $\phi \in C\left(\mu, X^{\prime}\right)$ that defines a parametric measure and it is $\epsilon$-lower bounded with respect to the (finite) $\mathbf{m}$ orthogonal sequence $\left\{f_{i}\right\}_{i=1}^{m}$. Let $\delta>0$. Then there is a simple function $\phi^{\prime} \in$ $C\left(\mu, X^{\prime}\right)$ such that

$$
\left\|\sum_{i=1}^{m} \alpha_{i}^{\phi} f_{i}-\sum_{i=1}^{m} \alpha_{i}^{\phi^{\prime}} f_{i}\right\|_{L^{1}(\mu)} \leq \delta
$$

and consequently,

$$
\left|E_{1}(g, \phi)-E_{1}\left(g, \phi^{\prime}\right)\right| \leq \delta .
$$

Proof. We just need to consider the following inequalities and apply Theorem 4.2.3.

$$
\begin{gathered}
\left\|\sum_{i=1}^{m} \alpha_{i}^{\phi} f_{i}-\sum_{i=1}^{m} \alpha_{i}^{\phi^{\prime}} f_{i}\right\|_{L^{1}(\mu)} \leq \int_{\Omega}\left(\sum_{i=1}^{m}\left(\alpha_{i}^{\phi}-\alpha_{i}^{\phi^{\prime}}\right)^{2}\right)^{\frac{1}{2}}\left(\sum_{i=1}^{m} f_{i}^{2}\right)^{\frac{1}{2}} d \mu \\
\leq\left(\int_{\Omega} \sum_{i=1}^{m}\left(\alpha_{i}^{\phi}-\alpha_{i}^{\phi^{\prime}}\right)^{2} d \mu\right)^{\frac{1}{2}}\left(\int_{\Omega} \sum_{i=1}^{m} f_{i}^{2} d \mu\right)^{\frac{1}{2}}
\end{gathered}
$$




\subsection{An example of approximation with finite $\mathrm{m}-$ orthogonal sequences}

In this section we develop an easy numerical example in order to show the nonlinear approximation that can be obtained with our procedure. Let $\mathbf{n}: \Sigma \rightarrow$ $X$ be a countably additive vector measure, and let $v$ be a finite control measure for $\mathbf{n}$ that is equivalent to it and $L^{1}(\mathbf{n}) \subseteq L^{1}(v)$.

Definition 4.3.1. We say that $\mathbf{n}$ is $v$-simply representable if there is a natural number $m \in \mathbb{N}$ and a simple function $\Psi(\omega):=\sum_{j=1}^{m} x_{j} \chi_{A_{j}}(\omega)$ such that $\left\{A_{j}\right.$ : $j=1, \ldots, m\}$ defines a (measurable) partition of $\Omega,\left\{x_{j}: j=1, \ldots, m\right\}$ is a linearly independent subset of $X$, and

$$
\mathbf{n}(A)=\int_{A} \Psi(\omega) d v(\omega)=\sum_{j=1}^{m} v\left(A \cap A_{j}\right) x_{j}, \quad A \in \Sigma .
$$

Note that in this case the integral of a function $f \in L^{1}(\mathbf{n})$ can be directly computed by means of the formula

$$
\int_{A} f d \mathbf{n}=\sum_{j=1}^{m}\left(\int_{A \cap A_{j}} f d v\right) x_{j}, \quad A \in \Sigma .
$$

If $A \in \Sigma$, we denote by $L^{2}\left(A, \Sigma_{\mid A}, v_{\mid A}\right)$ to the function space defined by means of the restriction of the measure space $(\Omega, \Sigma, v)$ to $A$. In particular, we can consider the functions of $L^{2}(v)$ as functions of $L^{2}\left(A, \Sigma_{\mid A}, v_{\mid A}\right)$.

Lemma 4.3.2. Let $\mathbf{n}$ be a $v$-simply representable vector measure and let $\left\{f_{i}\right\}_{i=1}^{\infty}$ be an $\mathbf{n}$-orthogonal sequence. Then this sequence belongs to the space $L^{2}(v)$ and is also orthogonal in it. Moreover, it is orthogonal in each space

$$
L^{2}\left(A_{j}, \Sigma_{\mid A_{j}}, v_{\mid A_{j}}\right), \quad j=1, \ldots, m \text {. }
$$

Proof. Since $\mathbf{n}$ is simply representable we can find a function $\Psi(\omega):=$ $\sum_{j=1}^{m} x_{j} \chi_{A_{j}}$ related to it that satisfies the conditions of Definition 4.3.1. A direct calculation leads to the equivalence of the norms $\|\cdot\|_{L^{2}(v)}$ and $\|\cdot\|_{L^{2}(\mathbf{n})}$, and then it is easy to show that $L^{2}(\mathbf{n})=L^{2}(v)$. Moreover, since the set $\left\{x_{j}: j=1, \ldots, m\right\}$ is linearly independent we can get for each $j=1, \ldots, m$ a functional $x_{j}^{\prime} \in X^{\prime}$ 
that satisfies that $\left\langle x_{j}, x_{j}^{\prime}\right\rangle=\tau_{j}>0$ and $\left\langle x_{i}, x_{j}^{\prime}\right\rangle=0$ for every $i \neq j$. Then, for every $i, j, k=1, \ldots, m$ such that $i \neq j$ we obtain

$$
\tau_{k} \int_{A_{k}} f_{i} f_{j} d v=\left\langle\int_{\Omega} f_{i} f_{j} d \mathbf{n}, x_{k}^{\prime}\right\rangle=\left\langle 0, x_{k}^{\prime}\right\rangle=0 .
$$

This gives the result.

The following lemma is a direct consequence of Lemma 4.3.2.

Lemma 4.3.3. Let $\mathbf{n}$ be a simply representable vector measure by means of a function $\Psi:=\sum_{j=1}^{m} x_{j} \chi_{A_{j}}$ that satisfies the conditions of Definition 4.3.1. Let $n \in \mathbb{N}$ and let $\left\{f_{i}\right\}_{i=1}^{n}$ be a $\mathbf{n}$-orthogonal sequence. Then the sequence $\left\{f_{i, j}\right\}_{i=1, j=1}^{n, m}$ of $L^{2}(v)$ given by the functions $f_{i, j}:=f_{i} \chi_{A_{j}}, i=1, \ldots, n, j=$ $1, \ldots, m$ is orthogonal.

Theorem 4.3.4. Let $\mathbf{n}, \Psi,\left\{f_{i}\right\}_{i=1}^{n}$ and $\left\{f_{i, j}\right\}_{i=1, j=1}^{n, m}$ as in Lemma 4.3.3. Then there is a Bochner integrable simple function $\Psi^{\prime}$ such that for every $g \in L^{2}(v)$ and every $i=1, \ldots, n$, the corresponding pointwise dependent Fourier coefficient satisfies the equality

$$
\alpha_{i}^{\Psi^{\prime}}(\omega) f_{i}(\omega)=\sum_{j=1}^{m} \alpha_{i, j} f_{i, j}(\omega),
$$

where the constants $\alpha_{i, j}=\frac{\int_{A_{j}} g f_{i} d v}{\int_{A_{j}} f_{i}^{2} d v}$ are the Fourier coefficients related to the functions $f_{i, j}$.

Consequently, the projection of the function $g$ on the subspace of $L^{2}(v)$ generated by $\left\{f_{i, j}\right\}_{i=1, j=1}^{n, m}$ is given by

$$
\sum_{i=1}^{n} \alpha_{i}^{\Psi^{\prime}}(\omega) f_{i}(\omega)
$$

Proof. The function $\Psi^{\prime}$ can be defined by means of the functionals $x_{j}^{\prime}$ that appear in the proof of Lemma 4.3.2. Using the elements introduced there, we define

$$
\Psi^{\prime}(\omega):=\sum_{j=1}^{m} x_{j}^{\prime} \chi_{A_{j}}(\omega) .
$$

Now we just need to compute for every index $i=1, \ldots, n$ the Fourier coefficient $\alpha_{i}^{\Psi^{\prime}}(\omega)$ by means of the corresponding straightforward calculation.

$$
\alpha_{i}^{\Psi^{\prime}}(\omega)=\frac{\left\langle\int_{\Omega} g f_{i} d \mathbf{n}, \Psi^{\prime}(\omega)\right\rangle}{\left\langle\int_{\Omega} f_{i}^{2} d \mathbf{n}, \Psi^{\prime}(\omega)\right\rangle}
$$




$$
=\frac{\sum_{j=1}^{m} \tau_{j}\left(\int_{A_{j}} g f_{i} d v\right) \chi_{A_{j}}}{\sum_{j=1}^{m} \tau_{j}\left(\int_{A_{j}} f_{i}^{2} d v\right) \chi_{A_{j}}}=\sum_{j=1}^{m}\left(\frac{\int_{A_{j}} g f_{i} d v}{\int_{A_{j}} f_{i}^{2} d v}\right) \chi_{A_{j}} .
$$

These equalities imply the result.

We finish this section with an example.

Example 4.3.5. Consider the 3 -dimensional Euclidean space $\ell_{2}^{3}$ and the vector measure $\mathbf{n}_{0}: \Sigma \rightarrow \ell_{2}^{3}$ given by

$$
\mathbf{n}_{0}(A):=\sum_{j=1}^{3} v_{0}(A \cap[j-1, j]) e_{j}, \quad A \in \Sigma,
$$

where $\left\{e_{i}\right\}_{i=1}^{3}$ is the basis of $\ell_{2}^{3}$ and $\left([0,3], \Sigma, v_{0}\right)$ is the Lebesgue measure space associated with the interval $[0,3]$. It is clear that $\mathbf{n}_{0}$ is a simply representable vector measure.

We consider the following polynomials. For every $x \in[0,3]$, we define

(1) $p_{1}(x):=1$,

(2) $p_{2}(x):=\frac{3}{2}-\frac{11}{2} x+\frac{9}{2} x^{2}-x^{3}$,

(3) $p_{3}(x):=\frac{171}{175}-\frac{63}{5} x+\frac{393}{10} x^{2}-\frac{252}{5} x^{3}+\frac{309}{10} x^{4}-9 x^{5}+x^{6}$.

Figure 4.1 shows the functions of this sequence. A direct calculation shows that

Figure 4.1: The functions of the $\mathbf{n}_{0}$-orthogonal sequence.

it defines a (finite) $\mathbf{n}_{0}$-orthogonal sequence of square $\mathbf{n}_{0}$-integrable functions. 
Let $g(x):=\frac{3.5}{10 x^{2}+1}+2 e^{\left(-3(x-1.5)^{2}\right)}$ be a function of $L^{2}\left(\mathbf{n}_{0}\right)$. Figure 4.2 shows this function.

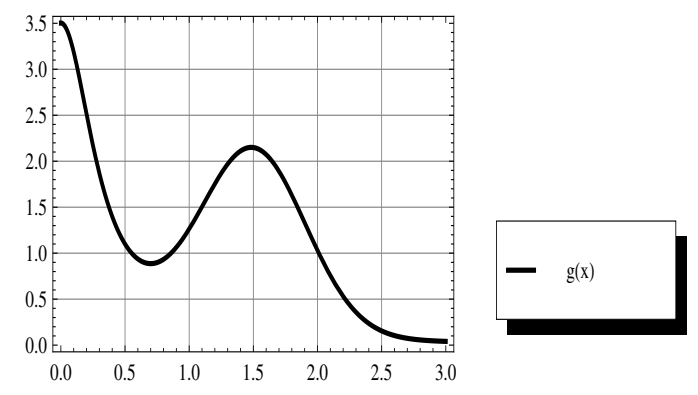

Figure 4.2: The function $g(x):=\frac{3.5}{10 x^{2}+1}+2 e^{\left(-3(x-1.5)^{2}\right)}$.

Let us consider different functions $\phi$ and compute the corresponding pointwise dependent Fourier coefficients $\alpha_{i}^{\phi}$. It is clear that in this case $L^{2}\left(\mathbf{n}_{0}\right)=$ $L^{2}\left(v_{0}\right)$.

Let $\phi_{1}(x):=\sum_{j=1}^{3} \chi_{[j-1, j]}(x) e_{j}$. This function satisfies the conditions of Theorem 4.3.4, and then the approximation given by the pointwise dependent Fourier coefficients

$$
\alpha_{i}^{\phi_{1}}(x)=\sum_{j=1}^{3} \frac{\int_{[j-1, j]} p_{i} g d v_{0}}{\int_{[j-1, j]} p_{i}^{2} d v_{0}} \chi_{[j-1, j]}(x),
$$

$i=1,2,3$, gives the approximation

$$
h_{1}(x):=\sum_{i=1}^{3} \alpha_{i}^{\phi_{1}}(x) p_{i}(x)
$$

to the function $g$ given by the projection on the subspace of $L^{2}\left(v_{0}\right)$ generated by the functions $p_{i, j}:=p_{i} \chi_{[j-1, j]}, i, j=1,2,3$. Therefore, note that this function is not necessarily continuous, since it is a linear combination of noncontinuous functions (see Figure 4.3). 


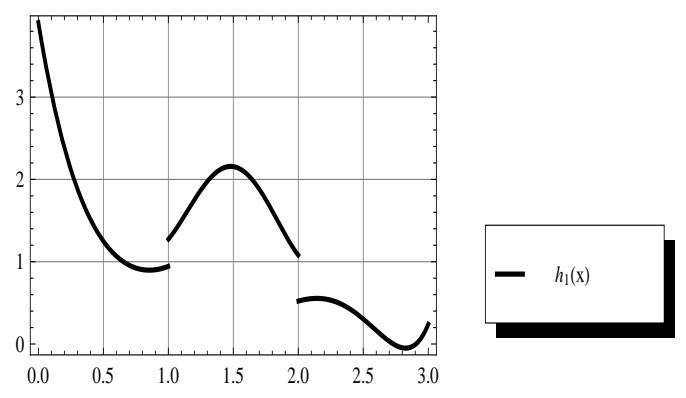

Figure 4.3: The function $h_{1}(x):=\sum_{j=1}^{3} \alpha_{i}^{\phi_{1}} p_{i}(x)$.

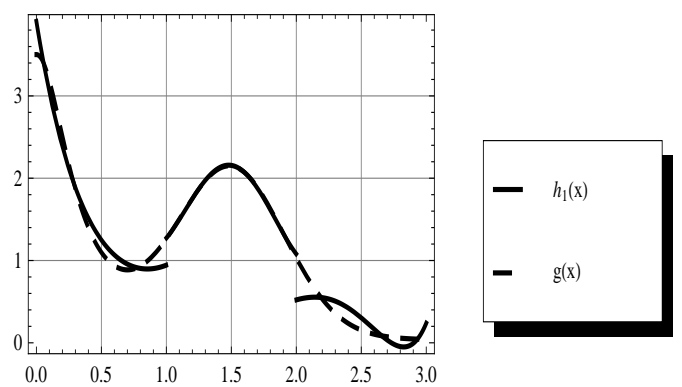

Figure 4.4: The function $g(x)$ and the approximation $h_{1}(x)$ using parametrization corresponding to $\phi_{1}(x)$. 
If we consider the function $\phi_{2}(x):=\chi_{[0,3]} \frac{\left(e_{1}+e_{2}+e_{3}\right)}{\sqrt{3}}$ and apply the same procedure (see Figure 4.5), we obtain a set of coefficients $\alpha_{i}^{\phi_{2}}, i=1,2,3$, that are constant functions. In fact, we obtain the coefficients of the standard approximation on the Hilbert space $L^{2}\left(v_{0}\right)$ by the functions $p_{1}, p_{2}$ and $p_{3}$,

$$
h_{2}(x)=\sum_{i=1}^{3} \alpha_{i}^{\phi_{2}} p_{i}(x)=1.22283 p_{1}(x)+0.735055 p_{2}(x)+0.104471 p_{3}(x) .
$$

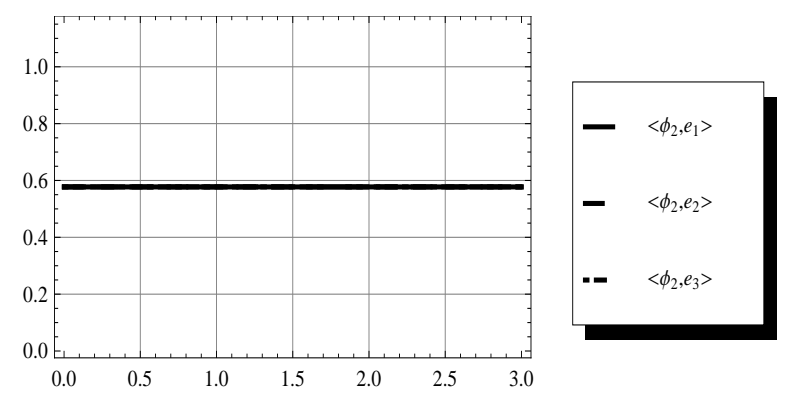

Figure 4.5: The graph of the projections of the function $\phi_{2}(x)$. All of them coincides.

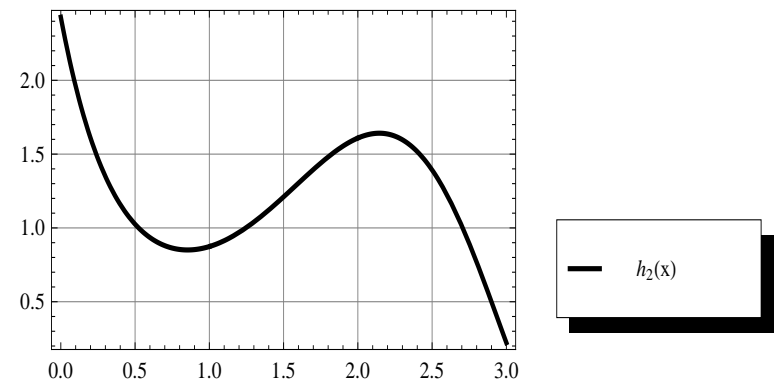

Figure 4.6: The function $h_{2}(x):=\sum_{j=1}^{3} \alpha_{i}^{\phi_{1}} p_{i}(x)$.

We can obtain a continuous function that approximate $g$ and is close to $h_{1}(x)$ just by applying the results of the previous point. Consider the function

$$
\psi(x):=\sum_{j=1}^{3} \exp \left(-\left(\frac{x-\left(j-\frac{1}{2}\right)}{0.4}\right)^{2}\right) e_{j},
$$


and define the function (see Figure 4.7)

$$
\phi_{3}(x):=\frac{\psi(x)}{\|\psi(x)\|}, \quad x \in[0,3] .
$$

In this case, the pointwise dependent Fourier coefficients that give the approximation $h_{3}(x)=\sum_{i=1}^{3} \alpha_{i}^{\phi_{3}}(x) p_{i}(x)$ are

$$
\alpha_{i}^{\phi_{3}}(x)=\frac{\sum_{j=1}^{3}\left(\int_{[j-1, j]} p_{i} g d v_{0}\right) \exp \left(-\left(\frac{x-\left(j-\frac{1}{2}\right)}{0.4}\right)^{2}\right)}{\sum_{j=1}^{3}\left(\int_{[j-1, j]} p_{i}^{2} d v_{0}\right) \exp \left(-\left(\frac{x-\left(j-\frac{1}{2}\right)}{0.4}\right)^{2}\right)}, \quad i=1,2,3 .
$$

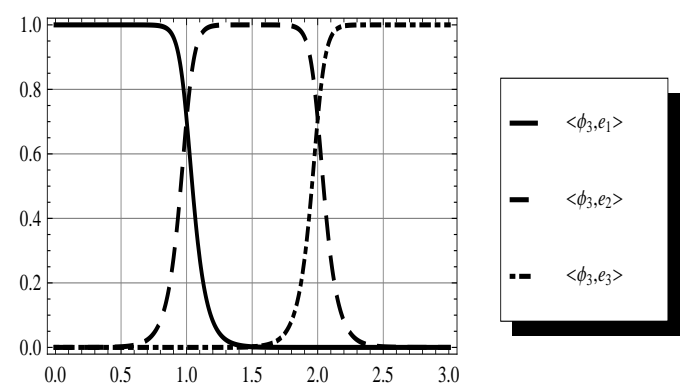

Figure 4.7: The graph of the projections of function $\phi_{3}(x)$.

Figure 4.8 shows $g(x)$ and the approximation $h_{3}(x)$ that is continuous by the construction.

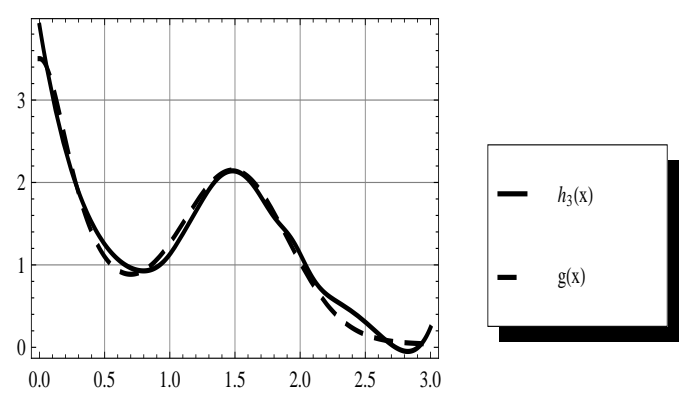

Figure 4.8: The function $g(x)$ and the approximations $h_{3}(x)$ using parametrization corresponding to $\phi_{3}(x)$.

The results on the continuity of the pointwise dependent Fourier coefficients can be applied to obtain the corresponding errors of this kind of approximation. 
If $\phi, \phi^{\prime} \in \mathcal{L}^{1}\left(v, X^{\prime}\right)$ satisfy that there is an $\epsilon>0$ such that both $\phi$ and $\phi^{\prime}$ are $\epsilon$-lower bounded with respect to the corresponding $\mathbf{n}$-orthogonal sequence (as in the case of the example), we can obtain bounds for the distances $\left\|\alpha_{i}^{\phi}-\alpha_{i}^{\phi^{\prime}}\right\|_{L^{2}(v)}$, in terms of $\left\|\phi-\phi^{\prime}\right\|_{L^{1}\left(v, X^{\prime}\right)}$ following the technique given in the proof of Theorem 4.2.3. Therefore, we can also obtain a bound for the $L^{1}(v)$-norm of the difference between the approximations associated to the corresponding functions $\phi$ and $\phi^{\prime}$, the 1-errors $E_{1}(g, \phi)$ and $E_{1}\left(g, \phi^{\prime}\right)$ and a bound for the difference between them using the procedure given in the proof of Corollary 4.2.5.

Remark 4.3.6. As a concluding remark, we want to point out that Figure 4.8 tries to show that our approach is fundamentally different from the classical one. This procedure - that weights every function $f_{i}$ with a Fourier coefficient that depends on the parametrized measure and it is in fact a function itself depending also on the variable - produces a non-linear approximation of the original function $g$. That is, consider that our function $g$ is the result of sampling a signal during an interval of time with length $\Delta T$ (in the precedent example, the interval is $[0,3]$, i. e., $\Delta T=3$. Then we choose functions $f_{i}$ (in the example $\left.\left\{p_{1}(x), p_{2}(x), p_{3}(x)\right\}\right)$ according with the shape of $g$ and we can produce the two approximations showed in the example. Suppose that these three functions are so suitable in shape that the Hilbert space approximation is very good. Now we sample again the signal and we obtain a new function $g$. If we are interested in keeping the same functions $f_{i}$, it is not guaranteed that the shape of them is suitable for this new approximation of $g$. In fact, Hilbert's approximation can be very bad. In our approach, parametrized Fourier coefficients avoid this and allow to keep the same bases of functions during iterative approximations. Consequently, our procedure is a natural framework for a dynamic approximation of functions depending on one parameter for example the time. In the following section, we show how this technique can be applied to a real problem.

\subsection{Application to acoustic data}

In the previous sections we have developed our approximation considering a function $g$ that mimics the true signals in some applied areas as it can be Physics. In this section we are going to present an analysis of a true signal coming from the field of Acoustics. In order to be consistent we are going to 
use the same sequence of functions that in the previous section. We will see that our technique is so flexible that functions that are not adequate "a priori "because its shape does not fit the shape of the signal, can be used in our case. Our approximation produces better results that the corresponding standard approximation.

We are going to use a particular example related to the Acoustics, more precisely to the Sonic Crystals. These are periodic arrangements of scatterers embedded in a medium with different physical properties -in our case cylinders of a rigid material embedded in air- [44]. These structures when are considered as an infinite periodic medium - eigenvalue problem- present ranges of frequencies where the transmission of waves is forbidden. These ranges are known as band gaps (BGs). In the last years, an increasing interest has appeared in the potential exploitation of SC as environmental noise barriers [56], [57]. However, the acoustical properties of SC depend on several factors showing some particularities in their attenuation properties. For example, the size and position of the BGs depend on several factors such as the direction of incidence of the wave on the SC and the type of arrangement of the scatterers [58]. As a consequence, the development of the screens based on SC is not a trivial process. An example of such type of structures can be seen in Figure 4.9.

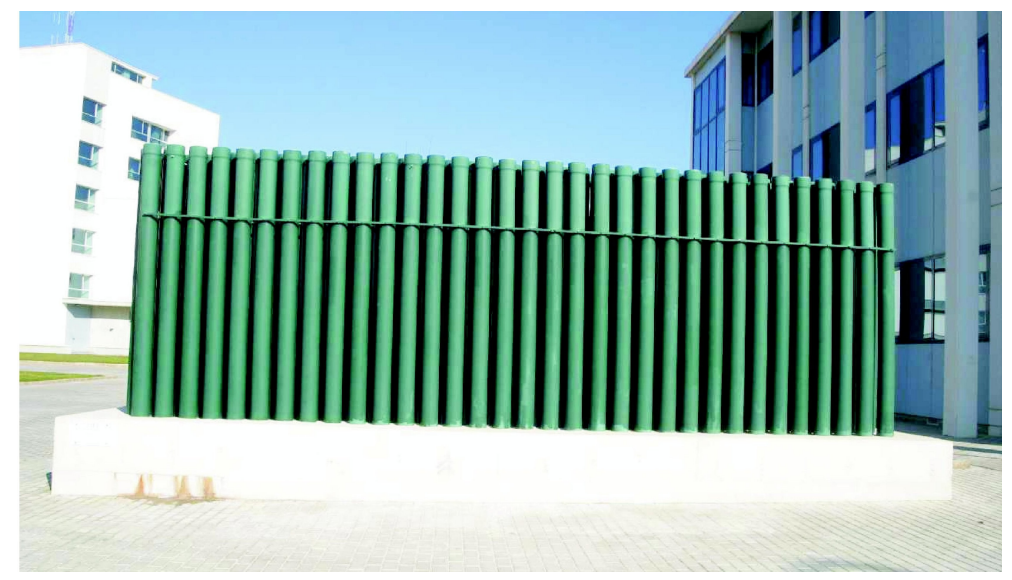

Figure 4.9: Example of Noise barrier based on a Sonic Crystal.

When a finite structure is considered the correct understanding of these Band Gaps implies Multiple Scattering Theory [12], [65]. Then waves are 
not transmitted throughout the structures due to the appearance of evanescent modes (see $[53,54,55]$ ). In this section we are dealing with one of these evanescent modes that are the modes that cannot be transmitted through the structure. The physical nature of such modes remains elusive and recent developments has allowed to clarify part of its nature. But the comparison of experimental measurements with theoretical models implies having good approximations of such a kind of signals. In this section we will try to show that orthogonal functions respect to vector measures could be an interesting tool in such kind of phenomena. If we measure the acoustic pressure between two rows of a SC, we observe a signal corresponding to an evanescent mode (see Figure 4.10). That is, a mode which amplitude decreases as the wave flies throughout the periodic structure.

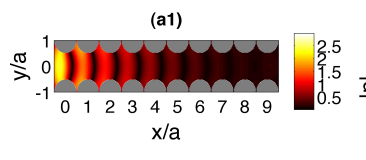

(b1)
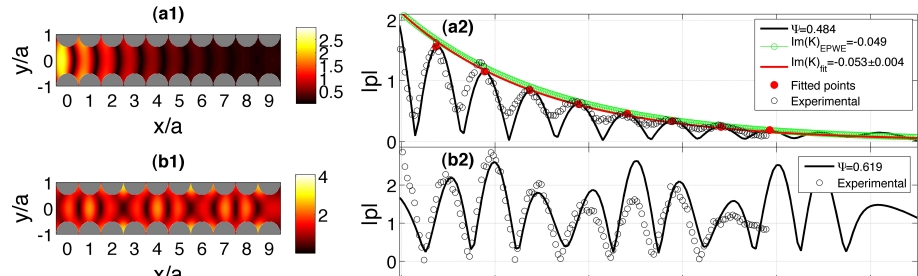

(c1)
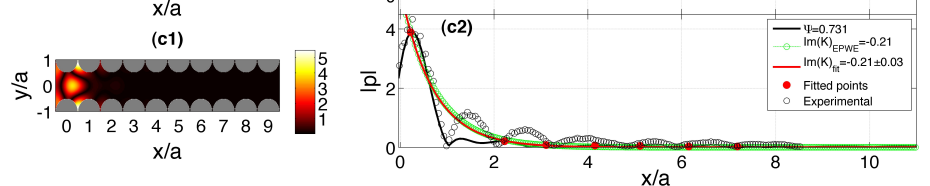

Figure 4.10: Measurement of normal modes $(b 1, b 2)$ in front of evanescent modes $(a 1, a 2)$ and $(c 1, c 2)$.

In order to make clear the comparison with the precedent approximations we are going to use the same finite sequence of $\mathbf{n}_{0}$-orthogonal functions ( $\mathbf{n}_{0}$ defined as before; see 4.3.5), shown in Figure 4.1 and also the same parametrizations $\phi_{2}(x)$, that provide the same coefficients than the standard approximation on the Hilbert space $L^{2}\left(v_{0}\right)$ by the functions $p_{1}, p_{2}$ and $p_{3}$, and $\phi_{3}(x)$. The signal to be approximated is represented in Figure 4.11.

If we apply our approximation with parametrization corresponding to function $\phi_{2}(x)$, we obtain the result shown in Figure 4.12. The result is not surprising because "a priori "the shape of the functions considered for our approximation is not the adequate for the shape of the signal, keeping apart the oscillations of 


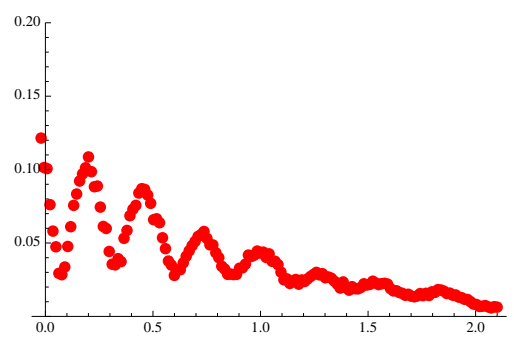

Figure 4.11: Data points of the signal to be approximated.

the signal. The approximation equivalent to the Hilbert's approximations it is not able to reproduce the decay of the original signal.

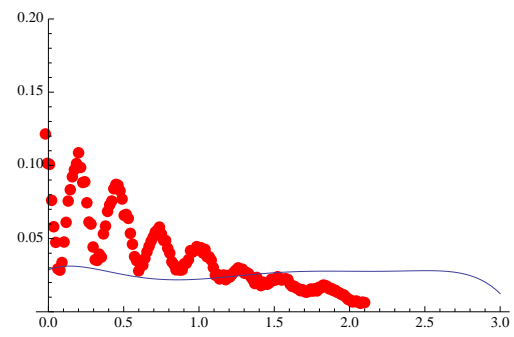

Figure 4.12: Hilbert approximation of the signal.

But in the case we consider the parametrization due to the $\phi_{3}(x)$, that allows us to weight the different behaviour of the signal in the support, then the result is much more satisfactory, keeping the same finite sequence of $\mathbf{n}_{0^{-}}$ orthogonal functions $\left\{p_{1}, p_{2}, p_{3}\right\}$. The result can be seen in Figure 4.13

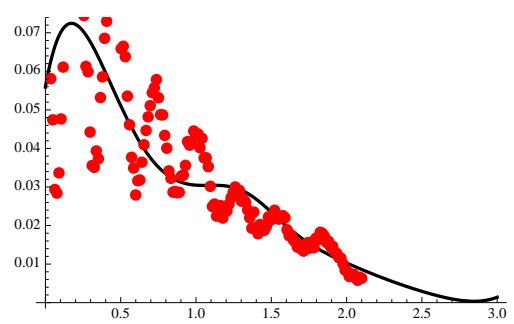

Figure 4.13: Vector Measure non-linear approximation.

As a summary if this section, it is clear that a much more suitable finite sequence of functions can be found in order to have a correct approximation 
from the Hilbert space point of view but, when a determined sequence have to be kept for doing the approximation of different signals which shape can change in different time intervals, this procedure based on the orthogonality respect to a vector measure is revealed as an interesting tool that provides approximations that reproduce correctly the shape of the original signal. 


\section{References}

[1] G. Alexits, Convergence problems of orthogonal series. Pergamon Press, New York, 1961.

[2] C. D. Aliprantis and O. Burkinshaw, Positive Operators, Academic Press, New York, 1985.

[3] C.D. Aliprantis and K.C. Border, Infinite dimensional analysis, Springer, Berlin, 1999.

[4] N. I. Akhiezer, The classical Moment Problem, Oliver and Boyd, London, 1965.

[5] R. G. Bartle, N. Dunford and J. T. Schwartz, Weak compactness and vector measures, Canad. J. Math. 7 (1955), 289-395 .

[6] G. Bennet, Unconditional convergence and almost everywhere convergence, Z. Wahrsch. Verw. Gebiete 34 (1976), 135-155.

[7] C. Bessaga, A. Pelczynski, On bases and unconditional convergence of series in Banach spaces, Studia Mathematica, 17 T.XVII.(1958), 151-164.

[8] C. Berg and G. Valent, The Nevanlinna parametrization for some indeterminate Stieltjes moment problems associated with birth and death processes, Methods Appl. Anal, 1 (1994), 169-209.

[9] J. M. Calabuig, J. Rodríguez and E. A. Sánchez Pérez, On the structure of $L^{1}$ of a vector measure via its integration operator, Integral Equations and Operator Theory 64 (2009), 21-33. 
[10] J. M. Calabuig, F. Galaz Fontes, E. Jiménez Fernández and E. A. Sánchez Pérez, Strong factorization of operators on spaces of vector measure integrable functions and unconditional convergence of series, Mathematische Zeitschrift 257 (2007), 381-402.

[11] L. Carleson, On convergence and growth of partial sums of Fourier series, Acta Math. 116 (1966), 135-157.

[12] Y. Chen and Zhen Ye Theoretical analysis of acoustic stop bands in twodimensional periodic scattering arrays Phys. Rev. E ,64, (2001), 036616.

[13] T. S. Chilara, On indeterminate Hamburger moment problem, pacific Journal of Mathematics. Vol. 27, No.3, 1968.

[14] G. Cohen and M. Lin, Extensions of the Menchoff-Rademacher theorem with applications to ergodic theory, Israel J. Math. 148,1 (2005), 41-86.

[15] G. P. Curbera, Banach space properties of $L^{1}$ of a vector measure, Proc. Amer. Math. Soc. 123(1995), 3797-3806.

[16] G. P. Curbera and W. J. Ricker, Banach lattices with the Fatou property and optimal domains of kernel operators, Indag. Math. (N.S.) 17 (2006), 187-204.

[17] G. P. Curbera and W. J. Ricker, Vector measures, integration and applications, in: Positivity, in: Trends Math., Birkhäuser, Basel (2007), 127-160.

[18] A. Defant, Variants of the Maurey-Rosenthal theorem for quasi Köthe function spaces, Positivity 5 (2001), 153-175.

[19] A. Defant and K. Floret, Tensor norms and operator ideals. North Holland Math. Studies, Amsterdam, 1993.

[20] A. Defant and M. Junge, Maximal theorems of Menchoff-Rademacher type in non-commutative $L_{q}$-spaces. J. Funct. Anal. 206 (2004), 322-355.

[21] A. Defant and M. Junge, Menchoff-Rademacher type theorems in vectorvalued Banach function spaces. Bull. Belg. Math. Soc. Simon Stevin 14, (2007), 473-482.

[22] R. del Campo, A. Fernández, I. Ferrando, F. Mayoral and F. Naranjo, Multiplication Operator on spaces of integrable functions with respect to a vector measure, J. Math. Anal. Appl. 343 (2008), 514-524. 
[23] R. del Campo and E. A. Sánchez Pérez, Positive representations of L1 of a vector measure, Positivity, 11 (2007), 449-459

[24] J. Diestel, Sequences and Series in Banach Spaces, Springer-Verlag New York, Inc. 1984.

[25] J. Diestel and J. J. Uhl, Vector Measures, Math. Surveys, vol. 15, Amer. Math. Soc., Providence, RI, 1977.

[26] J. Dieudonné, History of Functional Analysis, North Holland, Mathematics Studies, Amsterdam 1981.

[27] P. Enflo, Banach space with basis without a normalized monotone basis, Ark. Math, 1973.

[28] A. Fernández, F. Mayoral, F. Naranjo, C. Sáez and E.A. Sánchez-Pérez, Spaces of p-integrable functions with respect to a vector measure, Positivity 10 (2006), 1-16.

[29] A. Fernández, F. Mayoral, F. Naranjo, C. Sáez and E. A. Sánchez Pérez, Spaces of integrable functions with respect to a vector measure and factorizations through Lp and Hilbert spaces Journal of Mathematical Analysis and Applications, 330 (2007), 1249-1263

[30] I. Ferrando and E. A. Sánchez Pérez, Tensor product representation of the (pre)dual of the Lp-space of a vector measure, Journal of the Australian Mathematical Society 87 (2009), 211-225.

[31] I. Ferrando and J. Rodríguez, The weak topology on $L^{p}$ of a vector measure, Topology and its Applications 155 (2008), 1433-1444.

[32] J. Flores, Problemas de mayoración en clases de operadores entre retículos de Banach, Ph. D. Thesis Univ. Complutense de Madrid, 2001.

[33] D. H. Fremlin, Measure Theory, vol. 2: Broad Foundations, Torres Fremlin, Colchester, 2001.

[34] L.M. García-Raffi, D. Ginestar and E.A. Sánchez Pérez, Integration with respect to a vector measure and function approximation. Abst. Appl. Anal. 5,4 (2000), 207-226. 
[35] L.M. García-Raffi, D. Ginestar and E.A. Sánchez Pérez, Vector measure orthonormal systems and self-weighted functions approximation. Publications of RIMS. 41,3(2005), 551-563.

[36] L.M. García-Raffi, E.A. Sánchez Pérez, J.V. Sánchez Pérez, Commutative sequences of integrable functions and best approximation with respect to the weighted vector measure distance. Integral Equations and Operator Theory. 54(2006), 495-510.

[37] E. Jiménez Fernández, M. A. Juan and E.A. Sánchez-Pérez, A Komlós Theorem for abstract Banach lattices of measurable functions J. Math. Anal. Appl. DOI 10.1016/j.jmaa.2011.05.010.

[38] M.I. Kadec, A. Pelczynski, Lacunary sequences and complemented subspaces in the spaces $L^{p}$, Studia Mathematica, T.XXI. (1962), 161-176.

[39] M. Krein, D. Milman and M. Rutman, A note on basis in Banach spaces, Comm. Inst. Sci. Math. Méc. Univ. Kharkoff (4) 16 (1940), 106-110.

[40] D. R. Lewis, Integration with respect to vector measures, Pacific J. Math., 33 (1970), 157-165.

[41] D. R. Lewis, On integrability and summability in vector spaces, Illinois J. Math. 16, (1972), 294-307.

[42] J. Lindenstrauss and L. Tzafriri, Classical Banach Spaces II, Springer, Berlin, 1979.

[43] C. Linton, Mand Evans D.V. The interaction of waves with arrays of vertical circular cylinders J. Fluid Mech. 215 (1990), 549-569.

[44] R. Martínez-Sala ,J. Sancho, J.V. Sánchez-Pérez ,V. Gómez ,J . Llinares and F. Meseguer Sound attenuation by sculpture, Nature (1995), 378-241.

[45] D. Menšov, Sur les séries de fonctions orthogonales I, Fund. Math. 4 (1923), 82-105.

[46] D. Menšov, Sur les séries de fonctions orthogonales II, Fund. Math. 10 (1927), 375-420.

[47] R. Nevanlinna, Asymptotische Entwicklungen beschränkter Funktionen und das Stieltjessche Momentenproblem, Ann. Acad. Sci. Fenn. Ser. A (5) 18 (1922), 52 . 
[48] S. Oltra, E.A. Sánchez Pérez and O. Valero, Spaces $L_{2}(\lambda)$ of a positive vector measure $\lambda$ and generalized Fourier coefficients, Rocky Mountain Math. J. 35,1(2005), 211-224.

[49] S. Okada, W.J. Ricker and E.A. Sánchez Pérez, Optimal domain and integral extension of operators acting in function spaces. Operator Theory: Advances and Applications, 180. Birkhäuser Verlag, Basel, 2008.

[50] P. Ørno, A note on unconditionally converging series in $L_{p}$, Proc. Amer. Math. Soc. 59,2 (1976), 252-254.

[51] H. Rademacher, Einige Sätze über Reihen von allgemeinen Orthogonalfunktionen, Math. Ann. 87(1922), 112-138.

[52] M. Riesz, Sur le problème des moments et le théorème de Parseval correspondant, Acta Sci. Math. (Szeged) 1 (1923), 209-225.

[53] V. Romero-García, J.V. Sánchez-Pérez, S. Castiñeira Ibáñez, and L.M. Garcia-Raffi. Evidences of evanescent bloch waves in phononic crystals, Appl. Phys. Lett. 96, (2010), 124102.

[54] V. Romero-García, J.V. Sánchez-Pérez, and L.M. Garcia-Raffi. Modes in sonic crystals: Complex dispersion relation and supercell approximation, J. Appl. Phys. 108, (2010), 044907.

[55] V. Romero-García, J.V. Sánchez-Pérez, and L.M. Garcia-Raffi. Propagating and evanescent properties of double-point defects in sonic crystals, New. Jour. Phys. 12, (2010), 083024.

[56] J.V. Sánchez-Pérez, C. Rubio,R. Martínez-Sala,R. Sánchez-Grandia and V. Gómez, Acoustic barriers based on periodic arrays of scatterers Appl. Phys. Lett. 81 (2002) 5240.

[57] J. Sánchez-Dehesa,V. M. Garcia-Chocano,D. Torrent,F. Cerveraand S. Cabrera, Noise control by sonic crystal barriers made of recycled materials arXiv:1004.2570v1 [cond-mat.mtrl-sci] 2010.

[58] J.V. Sánchez-Pérez,D. Caballero,R. Martínez-Sala,C. Rubio,J. SánchezDehesa,F. Meseguer,J. Llinares and F. Gálvez,Sound Attenuation by a TwoDimensional Array of Rigid Cylinders, Phys. Rev. Lett. (1998) 80, 5325.

[59] E.A. Sánchez Pérez, Vector measure orthonormal functions and best approximation for the 4-norm. Arch. Math. 80(2003), 177-190. 
[60] E. A. Sánchez Pérez, Compactness arguments for spaces of p-integrable functions to a vector measure and factorization of operators through Lebesgue-Bochner spaces. Illinois J. Math. 453 (2001), 907-923.

[61] E. A. Sánchez Pérez, Vector measure duality and tensor product representation of Lp spaces of vector measures Proceedings of the American Mathematical Society, 132 (2004), 3319-3326.

[62] J. Stieltjes Recherches sur les fractions continues. Annales de la faculte des sciences de Toulousse, $1^{\mathrm{a}}$ serie, tome 8, $\mathrm{n}^{\mathrm{o}}$ 4, p J1-J122, 1894.

[63] G. Szegö, Orthogonal Polynomials, 4th ed. Providence, RI: Amer. Math. Soc., p. 33, 1975.

[64] P. Wojtaszczyk, Banach spaces for analysts, Cambridge University Press, Cambridge, 1991.

[65] F. Zaviska The deflection of electromagnetic waves on parallel, infinite long orbital cylinder Ann. Phys. 40 (1913), 1023-1056. 